\title{
Global existence results for viscoplasticity at finite strain*
}

\author{
Alexander Mielke ${ }^{\dagger}$, Riccarda Rossi ${ }^{\ddagger}$, Giuseppe Savaré ${ }^{\S}$
}

September 18, 2016

\begin{abstract}
We study a model for rate-dependent gradient plasticity at finite strain based on the multiplicative decomposition of the strain tensor, and investigate the existence of global-in-time solutions to the related PDE system. We reveal its underlying structure as a generalized gradient system, where the driving energy functional is highly nonconvex and features the geometric nonlinearities related to finite-strain elasticity as well as the multiplicative decomposition of finite-strain plasticity. Moreover, the dissipation potential depends on the left-invariant plastic rate and thus, depends on the plastic state variable.

The existence theory is developed for a class of abstract, nonsmooth, and nonconvex gradient systems, for which we introduce suitable notions of solutions, namely energy-dissipation-balance (EDB) and energy-dissipation-inequality (EDI) solutions. Hence, we resort to the toolbox of the direct method of the calculus of variations to check that the specific energy and dissipation functionals for our viscoplastic models comply with the conditions of the general theory.
\end{abstract}

\section{Introduction}

This paper is focused on the analysis of a model for elastoplasticity at finite strain in a bounded body $\Omega \subset \mathbb{R}^{d}$. Its elastic behavior is described by the deformation $\varphi: \Omega \rightarrow \mathbb{R}^{d}$. The ansatz at the core of finite-strain elastoplasticity, see [Lee69], is the multiplicative decomposition of the deformation gradient into an elastic and a plastic part, namely

$$
\nabla \varphi=F_{\mathrm{el}} F_{\mathrm{pl}} \doteq F_{\mathrm{el}} P,
$$

${ }^{*}$ A.M. has been partially supported by the ERC under AdG 267802 AnaMultiScale and by DFG under SFB 1114, project C5. R.R. and G.S. have been partially supported by a MIUR-PRIN'10-11 grant for the project "Calculus of Variations". R.R. also acknowledges support from the Gruppo Nazionale per l'Analisi Matematica, la Probabilità e le loro Applicazioni (GNAMPA) of the Istituto Nazionale di Alta Matematica (INdAM).

${ }^{\dagger}$ Weierstraß-Institut für Angewandte Analysis und Stochastik, Mohrenstraße 39, D-10117 Berlin, Germany. alexander.mielke@ wias-berlin.de

‡DIMI, Università di Brescia, via Valotti 9, I-25133 Brescia, Italy. riccarda.rossi @ unibs .it

${ }^{\S}$ Dipartimento di Matematica "F. Casorati", Università di Pavia. Via Ferrata, I-27100 Pavia, Italy. giuseppe . savare @ unipv . it 
which reflects the composition of elastic and plastic deformations. While the elastic part contributes to energy storage and is governed by an equilibrium equation, the plastic tensor $P$ evolves by a plastic flow rule.

The multiplicative decomposition (1.1) leads to significant geometric nonlinearities in the energy functional $\mathcal{J}=\mathcal{J}(t, \varphi, P)$ driving the evolution of the elastoplastic process. Nonetheless, such nonlinearities are compatible with polyconvexity of the energy density. In fact, the theory of polyconvex materials, dating back to [Bal77], has provided the analytical toolbox to handle elastostatics at finite strain. In particular, for the analysis of static microstructures in elastoplasticity we refer to, e.g., [M̈̈A91, OrR99, ORS00, CHM02, Mie03a, CoT05, CoO05.

A fundamental step towards the analysis of the evolution of finite-strain elastoplastic materials was made in OrS99]: therein it was pointed out that evolutionary elastoplastic models can be discretized by time-incremental problems that can be written as minimization problems for the sum of the dissipated and of the stored energy. This observation was mathematically formalized in [Mie03b] and forms the basis of the analysis in [MiM06, MaM09, HHM12, where the flow rule for the plastic tensor was considered rate-independent, i.e. driven by a dissipation potential that is positively homogenous of degree 1. In this context, the existence of (global) energetic solutions was proved by passing to the limit in a time-discretization scheme in the spirit of [OrS99]. In fact, the analysis developed in [FrM06, MaM09, MiR15a] shows that this solution concept for rateindependent processes is markedly suited to the geometric nonlinearities in finite-strain elastoplasticity. Essentially, its intrinsic variational character allows for the successful application of the direct methods in the calculus of variations also in the evolutionary case. Other mathematical results for energetic solutions of rate-independent material models at finite strain include crack propagation and brittle fracture, see [KZM10, DaL10].

In this paper we consider finite-strain elastoplasticity as a rate-dependent process, i.e. involving a dissipation potential with superlinear growth at infinity. A natural choice is of course given by a quadratic dissipation potential, leading to a classical gradient flow. In fact, our analysis builds on the by now well-established variational theory for gradient flows, see [Amb95, AGS05], and generalized gradient systems [RMS08, MRS13], which are characterized by nonquadratic dissipation potentials. In particular, along the footsteps of [MRS13] we will address two (intrinsically variational) solution concepts for the elastoplastic system, based on a suitable energy-dissipation inequality, which holds as an equality in the case of the strongest notion. Accordingly, we will obtain two distinct existence results.

Before illustrating our analysis more in detail, let us gain further insight into the features of the elastoplastic model under investigation, and in particular into the highly nonlinear driving energy.

\subsection{Modeling of viscoplasticity}

The elastoplastic evolution of the body $\Omega$ is described by two variables. The deformation $\varphi: \Omega \rightarrow \mathbb{R}^{d}$ is a mapping such that for almost all $x \in \Omega$ the gradient $\nabla \varphi(x)$ exists and belongs to the general linear group $\mathrm{GL}^{+}(d)$ of $(d \times d)$-matrices with positive determinant. 
Following the theory of generalized standard materials [HaN75, Mau92, Fré02], we consider the plastic tensor $P:=F_{\mathrm{pl}} \in \mathcal{P} \subset \mathrm{GL}^{+}(d)$, cf. (1.1), as an internal variable, modeling the internal state of the body. As such it is a macroscopic variable (we do not resolve the atomistic length), which is assumed to be generated by movements of dislocations, and maps the material frame (i.e. the crystallographic lattice) onto itself.

The evolution is governed by two principles:

Energy storage via a time-dependent Gibbs' free energy $\mathcal{J}(t, \varphi, P)$ and

Energy dissipation via a dissipation potential $\widehat{\Psi}(\varphi, P, \dot{\varphi}, \dot{P})$.

We assume that inertial effects can be ignored (quasistatic approximation) such that the equations of interest take the abstract variational form

$$
\begin{aligned}
& 0=\mathrm{D}_{\dot{\varphi}} \widehat{\Psi}(\varphi, P, \dot{\varphi}, \dot{P})+\mathrm{D}_{\varphi} \mathcal{J}(t, \varphi, P) \\
& 0 \in \partial_{\dot{P}} \widehat{\Psi}(\varphi, P, \dot{\varphi}, \dot{P})+\mathrm{D}_{P} \mathcal{J}(t, \varphi, P) .
\end{aligned}
$$

Here all derivatives should be taken as variational derivatives, and since $\mathcal{J}$ and $\widehat{\Psi}$ depend, except for potential external loadings, on the gradients $\nabla \varphi$ and $\nabla \dot{\varphi}$, the first equation (1.2a) has the usual divergence form, i.e. $\mathrm{D}_{\dot{\varphi}} \widehat{\Psi}$ contains the divergence of the viscous stresses while $\mathrm{D}_{\varphi} \mathcal{J}$ contains the divergence of the elastic stress tensor, see e.g. MaH94, Ant95, MOŞ14. Hence, (1.2a) provides the balance of linear momentum, whereas (1.2b) contains the plastic flow rule. The term $\mathrm{D}_{P} \mathrm{~J}$ contains the plastic backstress and the convex subdifferential $\partial_{\dot{P}} \widehat{\Psi}$ contains the viscoplastic stresses; in particular for $\dot{P}=0$ it features the yield stress. We refer to [Mie11] for general modeling background, even including a thermodynamically consistent modeling of temperature effects.

The theory of viscoelasticity at finite strain is notoriusly difficult and it seems that the present mathematical tools are not sufficient to provide sufficiently strong solutions in the multidimensional, truly geometrically invariant case, see the discussion in MOŞ14. Hence, we will neglect viscous effects subsequently by using the dissipation potential $\widehat{\Psi}(\varphi, P, \dot{\varphi}, \dot{P})=\Psi_{P}(\dot{P})$, which leads to a static equation for the displacement $\varphi$. This gives us the opportunity to replace the stationarity condition $\mathrm{D}_{\varphi} \mathcal{J}(t, \varphi, P)=0$ by the global minimality

$$
\varphi(t) \in \operatorname{Argmin}\{\mathcal{J}(t, \widetilde{\varphi}, P(t)): \widetilde{\varphi} \in \mathcal{F}\} .
$$

Thus, the starting point of our analysis is the system (1.3) and (1.2b) for the pair $(\varphi, P)$ : $[0, T] \rightarrow \mathcal{F} \times X$. However, the differential inclusion $(1.2 \mathrm{~b})$ has be replaced by a slightly weaker notion of solution, which we will call EDI solution or EDB solutions, see below.

The stored energy $\mathcal{J}$ and the dissipation potential $\Psi$ take the form

$$
\begin{aligned}
& \mathcal{J}(t, \varphi, P)=\int_{\Omega} \mathcal{W}(x, \nabla \varphi(x), P(x), \nabla P(x)) \mathrm{d} x-\langle\ell(t), \varphi\rangle \\
& \text { and } \Psi_{P}(\dot{P})=\int_{\Omega} \mathcal{R}(x, P(x), \dot{P}(x)) \mathrm{d} x,
\end{aligned}
$$

where $\ell$ is a sufficiently smooth time-dependent loading, see (3.L). For a given Banach space $X$, we continue to use $\langle\cdot, \cdot\rangle_{X}$ for the dual pairing on $X^{*} \times X$. 
The energy density $\mathcal{W}$ and the pointwise dissipation potential $\mathcal{R}$ feature geometric nonlinearities arising from frame indifference, non-self-interpenetration, and the Lie group structure of finite strains like the multiplicative decomposition (1.1). More precisely, this is reflected in a series of invariance principles for the energy density $\mathcal{W}$ and $\mathcal{R}$. First of all, we assume that $\mathcal{W}$ is given by the sum of an elastic part and of a part encompassing hardening and regularizing (through the gradient of the plastic variable) terms, i.e.

$$
\mathcal{W}(x, \nabla \varphi, P, \nabla P)=\mathcal{W}_{\mathrm{el}}(x, \nabla \varphi, P)+H(x, P, \nabla P)
$$

Following [MaH94, Ant95, Mie03b], the elastic part $W_{\text {el }}$ has to satisfy spatial frame indifference (or objectivity), namely

$$
\mathcal{W}_{\text {el }}(x, Q \nabla \varphi, P)=\mathcal{W}_{\text {el }}(x, \nabla \varphi, P) \quad \text { for all } Q \in \mathrm{SO}(d)
$$

i.e. invariance with respect to rotations acting from the left, which is compatible with polyconvexity together with the condition that $\mathcal{W}_{\mathrm{el}}(\nabla \varphi)=\infty$ for $\operatorname{det}(\nabla \varphi) \leq 0$, and $\mathcal{W}_{\mathrm{el}}(\nabla \varphi) \rightarrow \infty$ for $\operatorname{det}(\nabla \varphi) \downarrow 0$.

In addition, we postulate for $\mathcal{W}_{\mathrm{el}}$ and $\mathcal{R}$ plastic indifference (cf. [Mie03b]), namely

$$
\mathcal{W}_{\mathrm{el}}(x, \nabla \varphi \widetilde{P}, P \widetilde{P})=\mathcal{W}_{\mathrm{el}}(x, \nabla \varphi, P), \quad \mathcal{R}(x, P \widetilde{P}, \dot{P} \widetilde{P})=\mathcal{R}(x, P, \dot{P}) \quad \text { for } \widetilde{P} \in \mathcal{P} .
$$

This axiom implies that both $\mathcal{W}_{\text {el }}$ and $\mathcal{R}$ can be written in reduced form as

$$
\mathcal{W}_{\mathrm{el}}(x, \nabla \varphi, P)=W\left(x, \nabla \varphi P^{-1}\right), \quad \mathcal{R}(x, P, \dot{P})=R\left(x, \dot{P} P^{-1}\right) .
$$

These multiplicative structures give rise to strong geometric nonlinearities. This is easily seen when writing the PDE system induced by (1.3) and (1.2b) explicitly, namely

$$
\begin{gathered}
\varphi(t) \in \operatorname{Argmin}\left\{\int_{\Omega} W\left(x, \nabla \widetilde{\varphi}(x) P^{-1}(t, x)\right) \mathrm{d} x-\langle\ell(t), \widetilde{\varphi}\rangle: \widetilde{\varphi} \in \mathcal{F}\right\}, \\
\partial R\left(x, \dot{P} P^{-1}\right) P^{-\mathrm{\top}}+\left(\nabla \varphi P^{-1}\right)^{\top} \mathrm{D}_{F} W\left(x, \nabla \varphi P^{-1}\right) P^{-\mathrm{\top}} \\
+\mathrm{D}_{P} H(P, \nabla P)-\operatorname{div}\left(\mathrm{D}_{\nabla P} H(P, \nabla P)\right)=0,
\end{gathered}
$$

where $\mathcal{F}$ denotes the set of admissible deformations. Clearly, on the formal level (1.8a) yields solutions to (1.2a), as the latter is the Euler-Lagrange equation for the minimum problem in (1.8a). We refer to [ZR*06, Sec. 4] for an engineering application of such a finite-strain viscoplastic model, where $R(V)=\sigma_{\text {yield }}|V|+c|V|^{1.012}$, see also Example 3.5.

\subsection{Variational approaches: rate-independent versus rate-dependent evolution}

Variational approaches and formulations are ideal for treating material models involving finite-strain elasticity and finite-strain plasticity. The reason is that the direct methods from the calculus of variations rely on the flexible concept of weak lower semicontinuity, which allows us to circumvent the much too strong convexity methods that are available for small-strain theories. 
A first global existence result for finite-strain elastoplasticity was obtained in [MaM09], where solvability of (1.8) was addressed in the case of rate-independent systems, i.e. when $\widetilde{R}$ (and thus $R$ ) fulfills $\widetilde{R}(x, P, \lambda \dot{P})=\lambda \widetilde{R}(x, P, \dot{P})$ for every $\lambda \geq 0$. Indeed, the authors proved the existence of (global) energetic solutions to (1.8) according to the energetic concept (cf. MiR15b] for a general exposition), where $(\varphi, P):[0, T] \rightarrow \mathcal{F} \times X$ has to satisfy the (global) stability condition $(\underline{\mathrm{S}})$ and the energy balance $(\mathrm{E})$ for all $t \in[0, T]$ :

$$
\begin{aligned}
& \mathcal{J}(t, \varphi(t), P(t)) \leq \mathcal{J}(t, \widetilde{\varphi}, \widetilde{P})+\mathcal{D}(P(t), \widetilde{P}) \quad \text { for all }(\widetilde{\varphi}, \widetilde{P}) \in \mathcal{F} \times X, \\
& \mathcal{J}(t, \varphi(t), P(t))+\operatorname{Diss}_{\mathcal{D}}(P ;[0, t])=\mathcal{J}(0, \varphi(0), P(0))+\int_{0}^{t} \partial_{t} \mathcal{J}(s, \varphi(s), P(s)) \mathrm{d} s .
\end{aligned}
$$

Here $\mathcal{D}$ is an extended distance suitably defined from the 1-homogeneous dissipation $R$, and $\operatorname{Diss}_{\mathcal{D}}$ the induced dissipation functional. The most convenient feature of the energetic formulation via $(\underline{S})$ and $(\underline{E})$ is that it neither involves the pointwise derivative $\dot{P}$ of the plastic tensor, which is only BV as a function of time, nor any differential "DJ" of the energy $\mathcal{J}$. This is extremely advantageous in view of the highly nonlinear and nonsmooth character of $\mathcal{J}(t, \cdot, \cdot)$ and the fact that $(X, \mathcal{D})$ needs to be treated as a metric space not relying on a linear Banach space structure.

The present work is devoted to the rate-dependent case, but we still rely on the variational structure given by (1.2b) and (1.3), which form the abstract version of (1.8). Our system is induced by a generalized gradient system $(X, \mathcal{E}, \Psi)$ with the reduced energy $\mathcal{E}$ and the state-dependent dissipation potential $\Psi$ given by

$$
\mathcal{E}(t, P):=\inf \{\mathcal{J}(t, \varphi, P): \varphi \in \mathcal{F}\} \quad \text { and } \quad \Psi_{P}(\dot{P}):=\int_{\Omega} R\left(\dot{P} P^{-1}\right) \mathrm{d} x .
$$

Then, (1.8) can be rewritten as the abstract subdifferential inclusion

$$
0 \in \partial \Psi_{P(t)}(\dot{P}(t))+\mathfrak{F}(t, P(t)) \text { in } X^{*} \text { for a.a. } t \in(0, T),
$$

where the state space $X$ is $L^{p}\left(\Omega ; \mathbb{R}^{d \times d}\right)$, and the multivalued operator $\mathfrak{F}:[0, T] \times X \rightrightarrows X^{*}$ is the marginal subdifferential of $\mathcal{E}$ (cf. [MRS13]) defined via

$$
\mathfrak{F}(t, P):=\left\{\mathrm{D}_{P} \mathrm{~J}(t, \varphi, P): \varphi \text { is a minimizer for }(\underline{1.9})\right\} .
$$

Denoting by $\Psi_{P}^{*}$ the Fenchel-Moreau conjugate $\Psi_{P}^{*}(\Xi)=\sup \left\{\langle\Xi, V\rangle_{X}-\Psi_{P}(V): V \in\right.$ $X\}$ of $\Psi_{P}$, the celebrated Fenchel equivalence states that

$$
-\Xi \in \partial \Psi_{P}(\dot{P}) \quad \Longleftrightarrow \quad \Psi_{P}(\dot{P})+\Psi_{P}^{*}(-\Xi)=-\langle\Xi, \dot{P}\rangle_{X}
$$

Thus, together with the chain rule

$$
\frac{\mathrm{d}}{\mathrm{d} t} \mathcal{E}(t, P(t))=\partial_{t} \mathcal{E}(t, P(t))+\langle\Xi(t), \dot{P}(t)\rangle_{X} \text { for all } \Xi(t) \in \mathfrak{F}(t, P(t)) \text { for a.a. } t \in(0, T)
$$

(cf. Section 2 for details, in particular for the treatment of the nonsmoothness of $t \mapsto$ $\mathcal{E}(t, p)$ induced by (1.9)) we see that the pointwise subdifferential inclusion (1.10) is equivalent to the energy-dissipation balance

$(\mathrm{EDB})$

$$
\left\{\begin{array}{l}
\mathcal{E}(T, P(T))+\int_{0}^{T}\left(\Psi_{P(t)}(\dot{P}(t))+\Psi_{P(t)}^{*}(-\Xi(t))\right) \mathrm{d} t \\
=\mathcal{E}(0, P(0))+\int_{0}^{T} \partial_{t} \mathcal{E}(t, P(t)) \mathrm{d} t, \quad \text { where } \Xi(t) \in \mathfrak{F}(t, P(t)) .
\end{array}\right.
$$




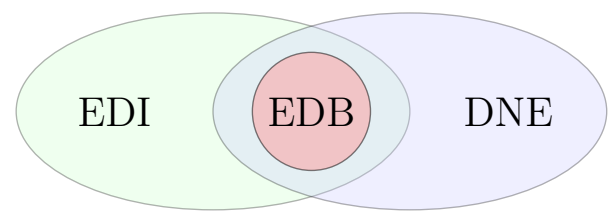

Figure 1.1: Schematic graph of solution concepts

In [Mie16] this equivalence is called the energy-dissipation principle, which has its origin in De Giorgi's theory of curves of maximal slope, cf. [DMT80].

Observe that both energy identities (E) and (1.13) balance the stored energy $\mathcal{E}$ with the work of the external forces and the dissipated energy. However, (1.13) features the additional dissipative term $\int_{0}^{T} \Psi_{P(r)}^{*}(-\Xi(r)) \mathrm{d} r$ (which is in fact null in the rate-independent case), involving the force term $\Xi(t)=\mathrm{D}_{P} \mathrm{~J}(t, \varphi(t), P(t))$ that needs to be suitably handled.

However, since the conjugate pair $\left(\Psi_{P}, \Psi_{P}^{*}\right)$ always satisfies the Young-Fenchel inequality $\Psi_{P}(V)+\Psi_{P}^{*}(\Xi) \geq\langle\Xi, V\rangle_{X}$ for all $V \in X$ and $\Xi \in X^{*}$, it is in fact sufficient to ask for the estimate " $\leq$ " in the right-hand side of (1.11). Thus, it suffices to replace the EDB by the weaker energy-dissipation inequality:

$$
(\text { EDI }) \quad\left\{\begin{array}{l}
\text { For } s=0 \text { and a.a. } s \in(0, T] \text { and all } t \in(s, T] \text { we have } \\
\mathcal{E}(t, P(t))+\int_{s}^{t}\left(\Psi_{P(r)}(\dot{P}(r))+\Psi_{P(r)}^{*}(-\Xi(r))\right) \mathrm{d} r \\
\leq \mathcal{E}(s, P(s))+\int_{s}^{t} \partial_{r} \mathcal{E}(r, P(r)) \mathrm{d} r, \quad \text { where } \Xi(r) \in \mathfrak{F}(r, P(r)) .
\end{array}\right.
$$

Assuming that the chain rule (1.12) holds, an easy application of the Young-Fenchel inequality gives the equivalence of EDI and EDB, see Proposition 2.6. However, in situations where the chain rule does not hold, EDI is strictly weaker. Thus, we can now define three different types of solutions for $P:[0, T] \rightarrow X$.

DNE solution: $P$ satisfies the doubly nonlinear eqn. $0 \in \partial \Psi_{P}(\dot{P})+\mathfrak{F}(t, P)$, see (1.10); EDI solution: $P$ satisfies the EDI (1.14);

EDB solution: $P$ satisfies the EDB (1.13) and the doubly nonlinear eqn. (1.10).

The exact form of EDI and EDB solutions are given in Definitions 2.1 and 2.4, respectively; see Figure 1.1 for the inclusion relations between the solution types. We will not address the notion of DNE solutions, which is typically used for evolutionary system formulated with monotone operators, since our approach involves the variational structure where the energy and dissipation are crucial to obtain a priori bounds.

\subsection{The main results}

For the analysis of the gradient system (1.10), with $\mathcal{E}$ and $\Psi$ given by (1.9), we will follow the abstract variational approach from [MRS13], which was developed exactly to treat nonsmooth and nonconvex energies like $\mathcal{E}$. There, following the spirit of the variational theory for gradient systems in metric spaces, a set of abstract conditions on 
the energy functional $\mathcal{E}$ and on the dissipation potential $\Psi$ were derived that guarantee to the existence of an energy solution (i.e. fulfilling the EDB (1.13)) to (1.10).

While referring for more details to Section 2, where the results from [MRS13] are recapitulated, let us highlight here the role of the required conditions for $(\mathcal{E}, \mathfrak{F}, \Psi)$ on coercivity, (lower semi-) continuity, compactness, and closedness. First we need compactness of sublevels of $\mathcal{E}$ and continuity of $\Psi_{P}(\cdot)$ to ensure that the time-incremental minimization problems

$$
P_{\tau}^{n} \in \underset{P \in X}{\operatorname{Argmin}}\left(\tau \Psi_{P_{\tau}^{n-1}}\left(\frac{P-P_{\tau}^{n-1}}{\tau}\right)+\mathcal{E}\left(\mathrm{t}_{\tau}^{n}, P\right)\right)
$$

with the state space $X=L^{p}\left(\Omega ; \mathbb{R}^{d \times d}\right)$, have solutions for every partition $\left\{0=\mathrm{t}_{\tau}^{0}<\mathrm{t}_{\tau}^{1}<\right.$ $\left.\cdots<\mathrm{t}_{\tau}^{N-1}<\mathrm{t}_{\tau}^{N}=T\right\}$ of $[0, T]$. Classical arguments from the theory of minimizing movements, cf. Amb95, AGS05 show that the approximate solutions constructed from $\left(P_{\tau}^{n}\right)_{\tau=0}^{N}$ satisfy a discrete version of the EDI (1.14). The limit passage $\tau \downarrow 0$ in this discrete EDI is ensured by the following closedness/continuity property for $(\mathcal{E}, \mathfrak{F})$ :

$$
\left.\begin{array}{ll}
P_{n} \rightarrow P & \text { in } X, \\
\Xi_{n} \rightarrow \Xi & \text { in } X^{*}, \\
\Xi_{n} \in \mathfrak{F}\left(t, P_{n}\right) & \text { for all } n \in \mathbb{N}
\end{array}\right\} \Longrightarrow \Xi \in \mathfrak{F}(t, P) \text { and } \mathcal{E}\left(t, P_{n}\right) \rightarrow \mathcal{E}(t, P)
$$

Hence, the limit $P$ for $\bar{P}_{\tau}(t) \rightarrow P(t)$ satisfies the time-continuous EDI (1.14) and the existence of EDI solutions follows, see Theorem 2.7. Moreover, if a suitable version of the chain rule is available we even have an EDB solution, see Theorem 2.7.

In Section 3 the viscoplastic model is described in full detail and and the main existence result for EDI solutions for the viscoplastic model (1.8) is stated, see Theorem 3.3. The proof of this result is the content of Section 4, where we check the assumptions of the abstract theory. Compactness of sublevels for the energy functional $\mathcal{E}$ is provided by hardening and a gradient of plasticity term in $\int_{\Omega} H(P, \nabla P) \mathrm{d} x$ with $H(P, A) \geq C\left(|P|^{q_{\mathrm{P}}}+1 /(\operatorname{det} P)^{q_{\gamma}}+|A|^{q_{\mathrm{G}}}\right)$. As in [MaM09, MiR15a], we require $q_{\mathrm{G}}>d$ to ensure $P \in W^{1, q_{\mathrm{G}}}\left(\Omega ; \mathbb{R}^{d \times d}\right) \subset \mathrm{C}^{0}\left(\bar{\Omega} ; \mathbb{R}^{d \times d}\right)$ as well as $P^{-1} \in \mathrm{C}^{0}\left(\bar{\Omega} ; \mathbb{R}^{d \times d}\right)$, which will play a crucial role for handling the multiplicative nonlinearities $\nabla \varphi P^{-1}$ and $\dot{P} P^{-1}$.

The closedness/continuity condition (1.16) is the most difficult part in the application to finite-strain plasticity, since the marginal subdifferential

$$
\mathfrak{F}(t, P)=\left\{-\operatorname{div}\left(\mathrm{D}_{\nabla P} H(P, \nabla P)\right)+\mathrm{D}_{P} H(P, \nabla P)+B(\nabla \varphi, P): \varphi \text { minimizes } \mathcal{J}(t, \cdot, P)\right\}
$$

contains the plastic backstress $B(F, P)=\left(F P^{-1}\right)^{\top} D_{F_{\mathrm{el}}} W\left(F P^{-1}\right) P^{-\top}$. For $\mathfrak{F}\left(t, P_{n}\right)$ this backstress depends nonlinearly on the deformation gradients $\nabla \varphi_{n}$, for which there is only little control, namely that it is a minimizer. First one can use a multiplicative stress control for the Mandel stress tensor, namely

$$
\left|F_{\mathrm{el}}^{\top} \mathrm{D}_{F_{\mathrm{el}}} W\left(x, F_{\mathrm{el}}\right)\right| \leq C W\left(x, F_{\mathrm{el}}\right)+C^{2},
$$

which provides an $L^{1}$ bound for $B\left(\nabla \varphi_{n}, P_{n}\right)$. Second, the weak convergence of this nonlinear quantity is obtained by a technique developed in [DFT05, e.g. Lem. 4.11] that relies only on the minimization property of $\varphi_{n}$ and the lower semicontinuity of $\mathcal{J}(t, \cdot, \cdot)$. 
In fact, the multiplicative stress control condition for the Mandel tensor in (1.17) and the related control of the Kirchhoff tensor, namely $\left|\partial_{F_{\mathrm{el}}} W\left(x, F_{\mathrm{el}}\right) F_{\mathrm{el}}^{\mathrm{\top}}\right| \leq C W\left(x, F_{\mathrm{el}}\right)+C^{2}$ where introduced in [Bal84, BOP91] to derive suitable variants of the Euler-Lagrange equations in finite-strain elasticity with polyconvex energy densities. Applications in rate-independent processes were developed in [FrM06, MaM09, KZM10, MiR15a, even to control the power term $\partial_{t} \mathcal{J}(t, \phi, P)$ in the case of time-dependent Dirichlet boundary conditions.

Based on these preparations we are then able to derive the existence of EDI solutions for the viscoplastic problem (1.8), see Theorem 3.3 .

To show that these solutions are even EDB solutions, we need to verify the chain rule (1.12) for our application in viscoplasticity. One major point is to gain enough control over $t \in \Xi(t)$ such that the duality pairing $\langle\Xi, \dot{P}\rangle_{X}$ can be manipulated. However, because of the highly nonconvex character of the energy $\mathcal{J}$, we are lacking individual control of the backstress contribution $\Xi_{B}(t):=B(\nabla \varphi(t), P(t))$, which is only in $L^{1}\left(\Omega ; \mathbb{R}^{d \times d}\right.$, and the hardening-regularizing contribution $\Xi_{H}(t):=\mathrm{D}_{P} H(P, \nabla P)-\operatorname{div}\left(\mathrm{D}_{\nabla P} H(P, \nabla P)\right)$ of $\Xi=\Xi_{B}+\Xi_{H}$. Ultimately, we are able to prove the chain rule (1.12) only in the case in which the duality pairings between $\dot{P}$ and both contributions $\Xi_{B}$ and $\Xi_{H}$ are well defined individually, cf. also Remark 4.11. So far the validity of (1.12) is seemingly an open problem for the energy functional (1.9).

In Section 5 we consider a regularized energy $\mathcal{E}_{\eta}(t, P)=\min \left\{\mathcal{J}_{\eta}(t, \varphi, P): \varphi \in \mathcal{F}\right\}$ with

$$
\widetilde{\mathcal{J}}(t, \varphi, P)=\mathcal{J}(t, \varphi, P)+\eta \int_{\Omega} \widetilde{W}(\nabla \varphi) \mathrm{d} x, \text { where }\left|W_{\mathrm{el}}\left(F_{\mathrm{el}}\right)\right|^{p^{\prime}} \leq C \widetilde{W}(F)+C^{2}
$$

The new density $\widetilde{W}$ provides a purely elastic part that gives higher integrability to $\nabla \varphi$ such that the backstress $\Xi_{B}$, which is given in terms of the Mandel tensor, now lies in $X^{*}=L^{p^{\prime}}\left(\Omega ; \mathbb{R}^{d \times d}\right)$. This construction allows us to establish the chain rule (1.12) for $\mathcal{E}_{\eta}$ and all EDI solutions are indeed EDB solutions, see Theorem 5.2.

Finally, in Section 6 we discuss some extensions of our existence results, in particular to the case of time-dependent Dirichlet loadings.

\section{Solution concepts and existence results for gener- alized gradient systems}

As mentioned in the introduction, our approach to the existence theory for the initial boundary value problem for (1.8) is based on the study of the abstract generalized gradient system

$$
0 \in \partial \Psi_{P(t)}(\dot{P}(t))+\mathfrak{F}(t, P(t)) \quad \subset X^{*} \quad \text { for a.a. } t \in(0, T),
$$

supplemented with the initial condition

$$
P(0)=P_{0} \in X
$$

where we assume throughout that $X$ is a separable reflexive Banach space. Moreover, for $\mathcal{E}, \Psi$, and $\mathfrak{F}$ we use the following notions and notations: 
1. The energy functional $\mathcal{E}:[0, T] \times X \rightarrow(-\infty, \infty]$ has proper domain $[0, T] \times \mathrm{D}$, with $\mathrm{D} \subset X$, it is bounded from below and lower semicontinuous.

2. The dissipation potential $\left(\Psi_{P}\right)_{P \in \mathrm{D}}$ is a Finsler family of non-negative, convex functionals on $X$, such that for every $P \in \mathrm{D}$ both $\Psi_{P}$ and its conjugate $\Psi_{P}^{*}$ have superlinear growth at infinity; then, $\partial \Psi_{P}: X \rightrightarrows X^{*}$ denotes the subdifferential of $\Psi_{P}$ in the sense of convex analysis.

3. The multivalued mapping $\mathfrak{F}:[0, T] \times \mathrm{D} \rightrightarrows X^{*}$ is such that for every $t \in[0, T]$ the mapping $\mathfrak{F}(t, \cdot): \mathrm{D} \rightrightarrows X^{*}$ is a suitable notion of a subdifferential for $\mathcal{E}(t, \cdot)$.

In fact, we are going to apply this approach to the reduced energy $\mathcal{E}:(t, P) \mapsto \min \mathcal{J}(t, \cdot, P)$ defined by minimizing out the deformation variable $\varphi$ from the original energy $\mathcal{J}$. Even if $\mathcal{J}$ is differentiable with respect to $t \in[0, T]$, the minimization with respect to $\varphi$ leads to nonsmoothness in $t$, because the functional $\mathcal{J}(t, \cdot, P)$ is nonconvex. Hence, the subdifferential $\mathfrak{F}$ of $\mathcal{E}$ as well as the time derivative of $t \mapsto \mathcal{E}(t, P)$ need to be handled carefully by resorting to suitable surrogates of the differentials of $\mathcal{E}$. In particular, following [MRS13] we need a coupling of the choice $\Xi \in \mathfrak{F}(t, P)$ and the time derivative. Thus as a surrogate of the power $\partial_{t} \mathcal{E}$ we define the power functional

$$
\mathfrak{P}: \operatorname{graph}(\mathfrak{F}) \rightarrow \mathbb{R} \quad \text { Borel-measurable }
$$

satisfying suitable properties given below. The typical choices for the marginal subdifferential $\mathfrak{F}$ and the associated power functional $\mathfrak{P}$ in case of $\mathcal{E}(t, P)=\min \mathcal{J}(t, \cdot, P)$ are

$$
\begin{aligned}
& \mathfrak{F}(t, P)=\left\{\mathrm{D}_{P} \mathcal{J}(t, \varphi, P): \varphi \in \mathcal{M}(t, P)\right\}, \quad \text { where } \mathcal{M}(t, P):=\operatorname{Argmin} \mathcal{J}(t, \cdot, P), \\
& \mathfrak{P}(t, P, \Xi)=\sup \left\{\partial_{t} \mathcal{J}(t, \varphi, P): \varphi \in \mathcal{M}(t, P), \Xi=\mathrm{D}_{P} \mathcal{J}(t, \varphi, P)\right\} .
\end{aligned}
$$

In the abstract setting of Section 2 we will not use this form, but in the application to

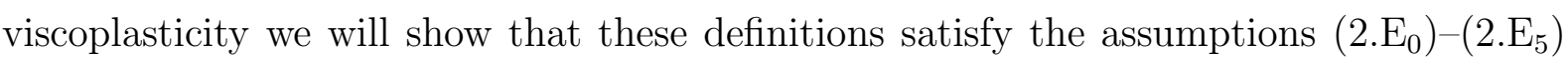
and $\left(2 . \Psi_{1}\right)-\left(2 . \Psi_{4}\right)$ given below.

In what follows, we shall refer to the quintuple $(X, \mathcal{E}, \Psi, \mathfrak{F}, \mathfrak{P})$ as a generalized gradient system, which gives rise to the generalized gradient-flow equation (2.1). We continue to use the short-hand $\Psi$ for the dissipation potential $\left(\Psi_{P}\right)_{P \in \mathrm{D}}$.

Next we will discuss three notions of solutions for $(X, \mathcal{E}, \Psi, \mathfrak{F}, \mathfrak{P})$ and a series of abstract conditions under which it is possible to obtain existence results for such concepts.

\subsection{Solution concepts}

Our first notion of solution consists of the energy-dissipation inequality (EDI) (2.8) below. As it will be clear from the the proof of Theorem 2.7 ahead, this is the weakest notion of solution arising in the limit of the Minimizing Movement scheme under the typical assumptions of lower semicontinuity, coercivity, and closedness on the pair $(\mathcal{E}, \Psi)$ in the variational approach to gradient flows, cf. [DMT80, Amb95, AGS05, RoS06, MRS13]. For our time-dependent situation we will need some extra condition on the power $\mathfrak{P}$. 
Definition 2.1 (EDI solution). We say that a function $P \in \mathrm{AC}([0, T] ; X)$ is a EDI solution (i.e. it satisfies the Energy-Dissipation Inequality) to the generalized gradient system $(X, \mathcal{E}, \Psi, \mathfrak{F}, \mathfrak{P})$ if there exist

1. a function $\mathscr{E} \in \mathrm{BV}([0, T])$ such that

$$
\begin{aligned}
& \mathscr{E}(t) \geq \mathcal{E}(t, P(t)) \text { for all } t \in[0, T], \text { and } \\
& \mathscr{E}(s)=\mathcal{E}(s, P(s)) \text { for a.a. } s \in(0, T) \text { and for } s=0
\end{aligned}
$$

2. a function $\Xi \in L^{1}\left(0, T ; X^{*}\right)$ with

$$
\Xi(t) \in \mathfrak{F}(t, P(t)) \quad \text { for a.a. } t \in(0, T) \text {; }
$$

such that the energy-dissipation inequality (EDI) holds for all $0 \leq s \leq t \leq T$

$$
(E D I) \quad \mathscr{E}(t)+\int_{s}^{t}\left(\Psi_{P(r)}(\dot{P}(r))+\Psi_{P(r)}^{*}(-\Xi(r))\right) \mathrm{d} r \leq \mathscr{E}(s)+\int_{s}^{t} \mathfrak{P}(r, P(r), \Xi(r)) \mathrm{d} r
$$

Remark 2.2. Clearly, from (2.6) and (2.8) we deduce

$$
\begin{aligned}
\mathcal{E}(t, P(t))+\int_{s}^{t}\left(\Psi_{P(r)}(\dot{P}(r))+\right. & \left.\Psi_{P(r)}^{*}(-\Xi(r))\right) \mathrm{d} r \\
\leq & \mathcal{E}(s, P(s))+\int_{s}^{t} \mathfrak{P}(r, P(r), \Xi(r)) \mathrm{d} r \\
& \quad \text { for all } t \in[0, T], \text { for } s=0, \text { and for a.a. } s \in(0, t) .
\end{aligned}
$$

Furthermore, since the triple $(P, \Xi, \mathscr{E})$ also fulfills the energy-dissipation inequality in the differential form

$$
\dot{\mathscr{E}}(t)-\mathfrak{P}(t, P(t), \Xi(t)) \leq-\Psi_{P(t)}(\dot{P}(t))-\Psi_{P(t)}^{*}(-\Xi(t)) \quad \text { for a.a. } t \in(0, T),
$$

taking into account (2.6), we infer the distributional inequality

$$
\frac{\mathrm{d}}{\mathrm{d} t} \mathcal{E}(t, P(t))+\Psi_{P(t)}(\dot{P}(t))+\Psi_{P(t)}^{*}(-\Xi(t)) \leq \mathfrak{P}(t, P(t), \Xi(t)) \quad \text { in } \mathscr{D}^{\prime}(0, T) .
$$

Remark 2.3 (Brenier's dissipative solutions). Our notion of EDI solutions is closely related to the notion of dissipative solutions introduced in [Bre15, Sec. 5.1]. There the dual dissipation potential $\Psi_{P}^{*}(-\Xi)$ is estimated from below which leads to the estimate

$$
\begin{array}{r}
\mathcal{E}(t, P(t))+\int_{0}^{t}\left(\Psi_{P(r)}(\dot{P}(r))-\langle\Xi(r), z(r)\rangle_{X}-\Psi_{P(r)}(z(r))\right) \mathrm{d} r \\
\leq \mathcal{E}(0, P(0))+\int_{0}^{t} \mathfrak{P}(r, P(r), \Xi(r)) \mathrm{d} r
\end{array}
$$

which is supposed to hold for a suitable dense set of test functions $z:[0, T] \rightarrow X$. The advantage in [Bre15] is that the difficult pairing $\langle\Xi(r), z(r)\rangle_{X}$ can be treated more efficiently for good test functions. 
All functions $P:[0, T] \rightarrow X$ satisfying the differential inclusion (2.1) are called $D N E$ solutions, since they solve the doubly nonlinear equation. Note that for EDI solutions, in general we are not able to establish (2.1), while DNE solutions need not fulfill EDI. We now present the much stronger concept of EDB solutions, which are contained in the intersection of EDI and DNE solutions, see Figure 1.1. Indeed we ask that EDI holds as an equality, namely the abstract Energy-Dissipation Balance (EDB) (2.12). In our abstract nonconvex setting, the validity of the doubly nonlinear differential inclusion (2.1) and of the EDB are strongly linked, which justifies our the choice of the following definition.

Definition 2.4 (EDB solution). We say that a function $P \in \mathrm{AC}([0, T] ; X)$ is an $\mathrm{EDB}$ solution to the generalized gradient system $(X, \mathcal{E}, \Psi, \mathfrak{F}, \mathfrak{P})$ if there exists $\Xi \in L^{1}\left(0, T ; X^{*}\right)$ such that the pair $(P, \Xi)$ solves the generalized gradient-flow equation (2.1), i.e.

$$
\Xi(t) \in \mathfrak{F}(t, P(t)) \quad \text { and } \quad 0 \in \partial \Psi_{P(t)}(\dot{P}(t))+\Xi(t) \quad \text { for almost all } t \in(0, T) \text {, }
$$

and fulfills, for all $0 \leq s \leq t \leq T$, the energy-dissipation balance

$$
(E D B) \quad\left\{\begin{aligned}
\mathcal{E}(t, P(t)) & +\int_{s}^{t}\left(\Psi_{P(r)}(\dot{P}(r))+\Psi_{P(r)}^{*}(-\Xi(r))\right) \mathrm{d} r \\
& =\mathcal{E}(s, P(s))+\int_{s}^{t} \mathfrak{P}(r, P(r), \Xi(r)) \mathrm{d} r .
\end{aligned}\right.
$$

Observe that the distributional inequality (2.10) reformulates as

$$
\Psi_{P(t)}(Q)-\Psi_{P(t)}(\dot{P}(t)) \geq \int_{\Omega}\langle-\Xi(t), Q\rangle_{X} \mathrm{~d} x+\frac{\mathrm{d}}{\mathrm{d} t} \varepsilon(t, P(t))-\mathfrak{P}(t, P(t), \Xi(t))
$$

for all $Q \in X$. This highlights the fact that the chain-rule inequality

$$
\frac{\mathrm{d}}{\mathrm{d} t} \mathcal{E}(t, P(t))-\mathfrak{P}(t, P(t), \Xi(t)) \geq \int_{\Omega}\langle\Xi(t), \dot{P}(t)\rangle_{X} \mathrm{~d} x,
$$

which would lead to the inequality

$$
\left.\Psi_{P(t)}(Q)-\Psi_{P(t)}(\dot{P}(t)) \geq \int_{\Omega}\langle-\Xi(t)), Q-\dot{P}(t)\right\rangle_{X} \mathrm{~d} x \text { for all } Q \in X,
$$

is in fact the missing ingredient to conclude from (2.13) that the pair $(P, \Xi)$ is a pointwise solution to (2.1) in the sense of (2.11). Indeed, our next result states that every EDI solution turns out to be an EDB solution if the functional $\mathcal{E}$ satisfies a suitable chain-rule inequality with respect to the triple $(\Psi, \mathfrak{F}, \mathfrak{P})$, namely

Definition 2.5 (Chain-rule inequality (CRI)). We say that the gradient system $(X, \mathcal{E}, \Psi, \mathfrak{F}, \mathfrak{P})$ satisfies the chain-rule inequality, if for every $P \in \mathrm{AC}([0, T] ; X)$ and $\Xi \in L^{1}\left(0, T ; X^{*}\right)$ with

$$
\begin{aligned}
& \sup _{t \in(0, T)}|\mathcal{E}(t, P(t))|<\infty, \quad \Xi(t) \in \mathfrak{F}(t, P(t)) \quad \text { for a.a. } t \in(0, T), \\
& \int_{0}^{T} \Psi_{P(t)}(\dot{P}(t)) \mathrm{d} t<\infty, \quad \text { and } \quad \int_{0}^{T} \Psi_{P(t)}^{*}(-\Xi(t)) \mathrm{d} t<\infty,
\end{aligned}
$$

we have that

$$
\text { (CRI) } \quad \frac{\mathrm{d}}{\mathrm{d} t} \mathcal{E}(t, P(t)) \geq\langle\Xi(t), \dot{P}(t)\rangle_{X}+\mathfrak{P}(t, P(t), \Xi(t)) \quad \text { for a.a. } t \in(0, T) \text {. }
$$


Proposition 2.6. Assume that $(X, \mathcal{E}, \Psi, \mathfrak{F}, \mathfrak{P})$ satisfies the $C R I$ (2.15). Then every EDI solution $P \in \mathrm{AC}([0, T] ; X)$ is an $E D B$ solution.

Proof. From (2.9) written for $s=0$ we obtain

$$
\begin{aligned}
& \mathcal{E}(t, P(t))+\int_{0}^{t}\left(\Psi_{P(r)}(\dot{P}(r))+\Psi_{P(r)}^{*}(-\Xi(r))\right) \mathrm{d} r \\
& \leq \mathcal{E}(0, P(0))+\int_{0}^{t} \mathfrak{P}(r, P(r), \Xi(r)) \mathrm{d} r \leq \mathcal{E}(t, P(t))+\int_{0}^{t}\langle-\Xi(r), \dot{P}(r)\rangle_{X} \mathrm{~d} r,
\end{aligned}
$$

where the latter estimate is due to the CRI (2.15). Thus, we get

$$
\int_{0}^{t}\left(\Psi_{P(r)}(\dot{P}(r))+\Psi_{P(r)}^{*}(-\Xi(r))-\langle-\Xi(r), \dot{P}(r)\rangle_{X}\right) \mathrm{d} r \leq 0 \quad \text { for every } t \in[0, T] .
$$

Since the integrand is non-negative by an elementary inequality from convex analysis, we conclude that $\Psi_{P(t)}(\dot{P}(t))+\Psi_{P(t)}^{*}(-\Xi(t))-\langle-\Xi(t), \dot{P}(t)\rangle_{X}=0$ for a.a. $t \in(0, T)$, whence $-\Xi(t) \in \partial \Psi_{P(t)}(\dot{P}(t))$ for a.a. $t \in(0, T)$. Taking into account (2.7), we conclude (2.11). The above arguments also yield that all inequalities in (2.16) hold as equalities for every $t \in(0, T]$, whence the EDB (2.12) .

\subsection{Assumptions for the abstract theory}

We now detail the exact conditions on the generalized gradient system $(X, \mathcal{E}, \Psi, \mathfrak{F}, \mathfrak{P})$, under which the existence of EDI solutions to the Cauchy problem for can be obtained via the techniques developed in [MRS13].

We define $\mathrm{D}(t):=\operatorname{dom} \mathcal{E}(t, \cdot)=\{P \in X: \mathcal{E}(t, P)<\infty\}$ and assume

$$
\mathrm{D}:=\mathrm{D}(0)=\mathrm{D}(t) \quad \text { for all } t \in[0, T] \text {. }
$$

Hereafter, we shall use the notation

$$
\mathcal{G}(P):=\inf _{t \in[0, T]} \mathcal{E}(t, P) \quad \text { for all } P \in \mathrm{D}
$$

For the energy functional $\mathcal{E}$ we require the following conditions, where we will use $C, C^{\prime}$, $C_{0}, K_{1}$ etc. for various positive constants depending only on known quantities.

\section{Lower semicontinuity:}

the map $P \mapsto \mathcal{E}(t, P)$ is lower semicontinuous for all $t \in[0, T]$,

$\exists C_{0}>0 \forall(t, u) \in[0, T] \times \mathrm{D}: \mathcal{E}(t, P) \geq \mathcal{G}(P) \geq C_{0}$, and

$\operatorname{graph}(\mathfrak{F})$ is a Borel set in $[0, T] \times X \times X^{*}$.

(Note that, if $\mathcal{E}$ is bounded from below, then we can suppose without loss of generality that it is bounded by a strictly positive constant.)

Coercivity: For all $t \in[0, T]$

$$
\text { the map } \quad P \mapsto \mathcal{E}(t, P) \text { has compact sublevels in } X \text {. }
$$


Variational sum rule: If for some $P_{*} \in X$ and $\tau>0$ the point $\bar{P}$ is a minimizer of $P \mapsto \mathcal{E}(t, P)+\tau \Psi_{P_{*}}\left(\left(P-P_{*}\right) / \tau\right)$, then $\bar{P}$ fulfills the Euler-Lagrange equation

$$
\exists \Xi \in \mathfrak{F}(t, \bar{P}): \quad-\Xi \in \partial \Psi_{P_{*}}\left(\left(\bar{P}-P_{*}\right) / \tau\right) .
$$

Lipschitz continuity with respect to $t \in[0, T]$ :

$$
\exists K_{1}>0 \forall P \in \mathrm{D} \forall t, s \in[0, T]: \quad|\mathcal{E}(t, P)-\mathcal{E}(s, P)| \leq K_{1} \mathcal{G}(P)|t-s| .
$$

Conditioned differentiability with respect to $t \in[0, T]$ : There exists a power functional $\mathfrak{P}: \operatorname{graph}(\mathfrak{F}) \rightarrow \mathbb{R}$, which is a Borel map and which satisfies

$$
\begin{aligned}
& \exists K_{2}>0 \quad \forall(t, P, \Xi) \in \operatorname{graph}(\mathfrak{F}): \\
& \liminf _{h \downarrow 0} \frac{1}{h}(\mathcal{E}(t+h, P)-\mathcal{E}(t, P)) \leq \mathfrak{P}(t, P, \Xi) \leq \lim _{h \downarrow 0} \frac{1}{h}(\mathcal{E}(t, P)-\mathcal{E}(t-h, P)) \leq K_{2} \mathcal{G}(P) \text {. }
\end{aligned}
$$

Weak closedness of $(\mathcal{E}, \mathfrak{F}, \mathfrak{P}):$ For all $t \in[0, T]$ and for all sequences $\left(P_{n}\right)_{n}$ in $X,\left(\Xi_{n}\right)_{n}$ in $X^{*}$ satisfying $\Xi_{n} \in \mathfrak{F}\left(t, P_{n}\right), \mathscr{E}_{n}=\mathcal{E}\left(t, P_{n}\right), \quad \mathscr{P}_{n}=\mathfrak{P}\left(t, P_{n}, \Xi_{n}\right)$ as well as

$$
P_{n} \rightarrow P \text { in } X, \quad \Xi_{n} \rightarrow \Xi \text { weakly in } X^{*}, \quad \mathscr{P}_{n} \rightarrow \mathscr{P} \text { and } \mathscr{E}_{n} \rightarrow \mathscr{E} \text { in } \mathbb{R},
$$

we have

$$
\Xi \in \mathfrak{F}(t, P), \quad \mathscr{P} \leq \mathfrak{P}(t, P, \Xi), \quad \text { and } \quad \mathscr{E}=\mathcal{E}(t, P) .
$$

Gronwall's lemma, $\left.2 . \mathrm{E}_{0}\right)$, and $\left.2 . \mathrm{E}_{3}\right)$ provide also an upper bound for $\mathcal{E}(t, P)$, namely

$$
\forall P \in \mathrm{D}: \quad \mathcal{G}(P) \leq \inf _{t \in[0, T]} \mathcal{E}(t, P) \leq \sup _{t \in[0, T]} \mathcal{E}(t, P) \leq \exp \left(K_{1} T\right) \mathcal{G}(P)
$$

Condition (2. $\mathrm{E}_{4}$ ) is fitted to the power functional $\mathfrak{P}$ that is given in (2.5), where only left and right derivatives are defined, see [KZM10, MRS13]. In simpler situations where $t \mapsto \mathcal{E}(t, P)$ is differentiable, we just have to use $\mathfrak{P}(t, P, \Xi)=\partial_{t} \mathcal{E}(t, P)$.

For the dissipation potentials $\left(\Psi_{P}\right)_{P \in \mathrm{D}}$ we require the following conditions:

Convexity and lower semicontinuity: For every $P \in \mathrm{D}$ the function

$$
\Psi_{P}: X \rightarrow[0, \infty) \text { is l.s.c., convex, and satisfies } \Psi_{P}(0)=0 \text {. }
$$

Superlinearity: The potentials $\left(\Psi_{P}\right)_{P \in \mathrm{D}}$ and $\left(\Psi_{P}^{*}\right)_{P \in \mathrm{D}}$ have uniform superlinear growth on sublevels of $\mathcal{E}$, viz.

$$
\forall S>0:\left\{\begin{array}{l}
\lim _{\|V\|_{X} \rightarrow \infty}\left(\frac{1}{\|V\|_{X}} \inf _{\mathcal{G}(P) \leq S} \Psi_{P}(V)\right)=\infty, \\
\lim _{\|\Xi\|_{X^{*} \rightarrow \infty}}\left(\frac{1}{\|\Xi\|_{X^{*}}} \inf _{\mathcal{G}(P) \leq S} \Psi_{P}^{*}(\Xi)\right)=\infty .
\end{array}\right.
$$

Mosco continuity: The map $P \mapsto \Psi_{P}$ is continuous on sublevels of $\mathcal{E}$ in the sense of Mosco convergence (cf. [Att84]), i.e. for all $S>0$ we have

$$
\left.\begin{array}{c}
P_{n} \rightarrow P \text { in } X, \quad \mathcal{G}\left(P_{n}\right) \leq S, \\
V_{n} \rightarrow V \text { in } X, \quad \Xi_{n} \rightarrow \Xi \text { in } X^{*}
\end{array}\right\} \Longrightarrow\left\{\begin{array}{c}
\liminf _{n \rightarrow \infty} \Psi_{P_{n}}\left(V_{n}\right) \geq \Psi_{P}(V), \\
\liminf _{n \rightarrow \infty} \Psi_{P_{n}}^{*}\left(\Xi_{n}\right) \geq \Psi_{P}^{*}(\Xi) .
\end{array}\right.
$$


Univaluedness of $\Psi_{P}^{*}$ : For all $P \in \mathrm{D}$ we have

$$
\forall V \in X \forall \Xi_{1}, \Xi_{2} \in \partial \Psi_{P}(V): \quad \Psi_{P}^{*}\left(\Xi_{1}\right)=\Psi_{P}^{*}\left(\Xi_{2}\right) .
$$

As pointed out in [MRS13, Rem. 2.1], condition (2. $\Psi_{4}$ is satisfied if $\Psi_{P}$ is given by the sum of positively homogeneous or differentiable, convex potentials.

\subsection{Existence of EDI and EDB solutions}

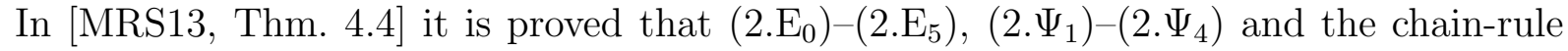
inequality CRI (2.15) imply the existence of EDB solutions for the Cauchy problem (2.1)(2.2). However, for the application to the viscoplasticity system (1.8), it will be crucial to establish an independent result on the existence of EDI solutions, cf. Theorem 2.7, because we are not able to establish the CRI in the general case. Although its proof can be inferred from the argument for [MRS13, Thm. 4.4], we will briefly outline it for the sake of readability.

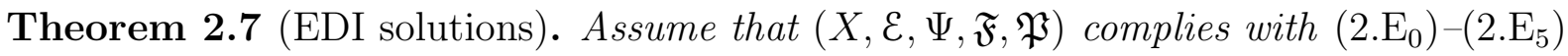
and $\left.2 . \Psi_{1}-2 . \Psi_{4}\right)$. Then, for every $P_{0} \in \mathrm{D}$ there exists an EDI solution to the Cauchy problem (2.1) $-(2.2)$.

As a straightforward consequence of Proposition 2.6 we have the following result.

Corollary 2.8 (EDB solutions, cf. [MRS13, Thm.4.4]). If additionally to the assumption of Theorem 2.7 the CRI (2.15) holds, then for every $P_{0} \in \mathrm{D}$ there exists an EDB solution to the Cauchy problem (2.1) -(2.2).

Sketch of the proof of Thm. 2.7. First of all, we set up the time-discretization scheme for (2.1): Given a partition $\left\{0=\mathrm{t}_{\tau}^{0}<\mathrm{t}_{\tau}^{1}<\ldots<\mathrm{t}_{\tau}^{N}=T\right\}$ of the interval $[0, T]$, with constant time-step $\tau=T / N$, we construct discrete solutions $\left(P_{\tau}^{n}\right)_{n=0}^{N} \subset X$ starting from $P_{\tau}^{0}:=P_{0}$ and finding

$$
P_{\tau}^{n} \in \underset{P \in X}{\operatorname{Argmin}}\left(\tau \Psi_{P_{\tau}^{n-1}}\left(\frac{P-P_{\tau}^{n-1}}{\tau}\right)+\mathcal{E}\left(\mathrm{t}_{\tau}^{n}, P\right)\right) .
$$

We thus define the approximate solutions to (2.1) via interpolation of the values $\left(P_{\tau}^{n}\right)_{n=0}^{N}$, with

$$
\left\{\begin{array}{lll}
\bar{P}_{\tau}(t):=P_{\tau}^{n} & \text { for } t \in\left(\mathrm{t}_{\tau}^{n-1}, \mathrm{t}_{\tau}^{n}\right], \\
\underline{P}_{\tau}(t):=P_{\tau}^{n-1} & \text { for } t \in\left[\mathrm{t}_{\tau}^{n-1}, \mathrm{t}_{\tau}^{n}\right), \\
P_{\tau}(t):=\frac{t-\mathbf{t}_{\tau}^{n-1}}{\tau} P_{\tau}^{n}+\frac{\mathrm{t}_{\tau}^{n}-t}{\tau} P_{\tau}^{n-1} & \text { for } t \in\left[\mathrm{t}_{\tau}^{n-1}, \mathrm{t}_{\tau}^{n}\right] .
\end{array}\right.
$$

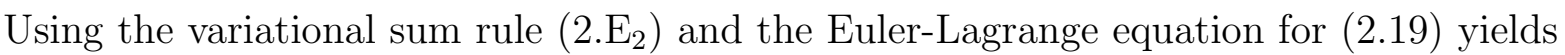

$$
0 \in \partial \Psi_{\underline{P}_{\tau}(t)}\left(\dot{P}_{\tau}(t)\right)+\mathfrak{F}\left(\overline{\mathrm{t}}_{\tau}(t), \bar{P}_{\tau}(t)\right) \quad \subset X^{*} \quad \text { for a.a. } t \in(0, T),
$$

where $\overline{\mathrm{t}}_{\tau}$ denotes the piecewise constant interpolant associated with the partition.

Due to the lack of convexity of $\mathcal{E}(t, \cdot)$, it turns out that the approximate version (2.20) of (2.1) is not sufficient for the limit passage to the time-continuous level. One needs the 
finer information provided by the approximate EDI

$$
\begin{aligned}
& \mathcal{E}\left(\overline{\mathrm{t}}_{\tau}(t), \bar{P}_{\tau}(t)\right)+\int_{\overline{\mathrm{t}}_{\tau}(s)}^{\overline{\mathrm{t}}_{\tau}(t)}\left(\Psi_{\underline{P}_{\tau}(r)}\left(\dot{P}_{\tau}(r)\right)+\Psi_{\underline{P}_{\tau}(r)}^{*}\left(-\widetilde{\Xi}_{\tau}(r)\right)\right) \mathrm{d} r \\
& \leq \mathcal{E}\left(\overline{\mathrm{t}}_{\tau}(s), \bar{P}_{\tau}(s)\right)+\int_{\overline{\mathrm{t}}_{\tau}(s)}^{\overline{\mathrm{\tau}}_{\tau}(t)} \mathfrak{P}\left(r, \widetilde{P}_{\tau}(r), \widetilde{\Xi}_{\tau}(r)\right) \mathrm{d} r
\end{aligned}
$$

for every $0 \leq s \leq t \leq T$. Its proof (cf. [MRS13, Lemma 6.1]) relies on condition (2. $\Psi_{4}$ ) and on De Giorgi's variational interpolant for the values $\left(P_{\tau}^{n}\right)_{n=1}^{N}$ (cf. [Amb95, AGS05]), which is defined via $\widetilde{P}_{\tau}(0):=P_{0}$ and

$$
\widetilde{P}_{\tau}(t) \in \underset{P \in X}{\operatorname{Argmin}}\left(\left(t-\mathrm{t}_{\tau}^{n-1}\right) \Psi_{P_{\tau}^{n-1}}\left(\frac{P-P_{\tau}^{n-1}}{t-\mathrm{t}_{\tau}^{n-1}}\right)+\mathcal{E}(t, P)\right) \quad \text { for } t \in\left(t_{\tau}^{n-1}, t_{\tau}^{n}\right) .
$$

Then, $\widetilde{\Xi}_{\tau}:(0, T) \rightarrow X^{*}$ in (2.21) is a measurable selection with $\widetilde{\Xi}_{\tau}(t) \in \mathfrak{F}\left(t, \widetilde{P}_{\tau}(t)\right)$ for a.a. $t \in(0, T)$ and satisfying the Euler-Lagrange equation for the minimum problem (2.22).

Continuing from (2.21) and exploiting the positivity of $\Psi$ and $\Psi^{*}$, one derives a series of a priori estimates for the families $\left(\bar{P}_{\tau}\right)_{\tau},\left(P_{\tau}\right)_{\tau},\left(\widetilde{P}_{\tau}\right)_{\tau}$ and $\left(\widetilde{\Xi}_{\tau}\right)_{\tau}$, namely

$$
\sup _{t \in[0, T]}\left(\mathcal{G}\left(\bar{P}_{\tau}(t)\right)+\mathcal{G}\left(\widetilde{P}_{\tau}(t)\right)\right) \leq C, \quad \int_{0}^{T}\left(\Psi_{\underline{P}_{\tau}(r)}\left(\dot{P}_{\tau}(r)\right)+\Psi_{\underline{P}_{\tau}(r)}^{*}\left(-\widetilde{\Xi}_{\tau}(r)\right)\right) \mathrm{d} r \leq C
$$

for a positive constant $C$, uniformly with respect to $\tau>0$. Hence, there exist $P \in$ $\mathrm{AC}([0, T] ; X)$ and $\widetilde{\Xi} \in L^{1}\left(0, T ; X^{*}\right)$ such that along a sequence $\tau_{k} \downarrow 0$ we have

$$
\begin{array}{ll}
\bar{P}_{\tau_{k}}, P_{\tau_{k}}, \widetilde{P}_{\tau_{k}} \rightarrow P & \text { in } L^{\infty}(0, T ; X), \\
\dot{P}_{\tau_{k}} \rightarrow \dot{P} & \text { in } L^{1}(0, T ; X), \\
\widetilde{\Xi}_{\tau_{k}} \rightarrow \widetilde{\Xi} & \text { in } L^{1}\left(0, T ; X^{*}\right)
\end{array}
$$

where the strong convergence with values in $X$ derives from the energy bound in (2.23) and the compactness of energy sublevels (cf. (2.E 10$)$, while (2.24b) $-(2.24 \mathrm{c})$ are a consequence of the integral bound of the dissipative terms $\Psi_{\underline{P}_{\tau}(r)}\left(\dot{P}_{\tau}(r)\right)$ and $\Psi_{\underline{P}_{\tau}(r)}^{*}\left(-\widetilde{\Xi}_{\tau}(r)\right)$, and of the superlinear growth $\left(2 . \Psi_{2}\right)$ of $\Psi$ and $\Psi^{*}$. From (2.23) it also follows that $\sup _{t \in(0, T)}\left|\mathfrak{P}\left(t, \widetilde{P}_{\tau}(t), \widetilde{\Xi}_{\tau}(t)\right)\right| \leq C$ via $\left.2 . \mathrm{E}_{4}\right)$. Moreover, Helly's principle also yields that there exists a function $\mathscr{E} \in \mathrm{BV}([0, T])$ such that

$$
\lim _{\tau_{k} \downarrow 0} \mathcal{E}\left(\overline{\mathrm{t}}_{\tau_{k}}(t), \bar{P}_{\tau_{k}}(t)\right)=\mathscr{E}(t) \quad \text { for every } t \in[0, T] .
$$

Taking into account that $\lim _{\tau_{k} \downarrow 0} \mathcal{E}\left(\overline{\mathrm{t}}_{\tau_{k}}(t), \bar{P}_{\tau_{k}}(t)\right) \geq \mathcal{E}(t, P(t))$ by the lower semicontinuity of the map $t \mapsto \mathcal{E}(t, P)$, combined with the fact that $\left|\mathcal{E}\left(\overline{\mathrm{t}}_{\tau_{k}}(t), \bar{P}_{\tau_{k}}(t)\right)-\mathcal{E}\left(t, \bar{P}_{\tau_{k}}(t)\right)\right| \rightarrow 0$ thanks to $\left.2 . \mathrm{E}_{3}\right)$, we deduce the first inequality in (2.6) .

To pass to the time-continuous limit it is necessary to gain further insight into the limiting properties of the sequences $\left(\widetilde{\Xi}_{\tau_{k}}\right)_{\tau_{k}}$ and $\left(\mathfrak{P}\left(t, \widetilde{P}_{\tau_{k}}, \widetilde{\Xi}_{\tau_{k}}\right)\right)_{\tau_{k}}$ by exploiting a Young-measure argument, see [MRS13, App. A]. A (not relabeled) subsequence $\left(\widetilde{\Xi}_{\tau_{k}}, \mathfrak{P}\left(t, \widetilde{P}_{\tau_{k}}, \widetilde{\Xi}_{\tau_{k}}\right)\right)_{\tau_{k}}$ possesses a limiting Young measure $\boldsymbol{\mu}=\left(\mu_{t}\right)_{t \in(0, T)}$, such that for almost all $t \in(0, T)$ the probability measure $\mu_{t}$ on $X^{*} \times \mathbb{R}$ is concentrated on the set of the weak limit points of the 
sequence $\left(\widetilde{\Xi}_{\tau_{k}}(t), \mathfrak{P}\left(t, \widetilde{P}_{\tau_{k}}(t), \widetilde{\Xi}_{\tau_{k}}(t)\right)\right)_{\tau_{k}}$. Taking into account the weak closedness property (2.E5) it follows that for almost all $t \in(0, T)$ we have $\mu_{t}\left(\left(X^{*} \times \mathbb{R}\right) \backslash \mathcal{S}(t, P(t))\right)=0$, where

$$
\mathcal{S}(t, P(t))=\left\{(\Xi, \mathfrak{p}) \in X^{*} \times \mathbb{R}: \Xi \in \mathfrak{F}(t, P(t)), \mathfrak{p} \leq \mathfrak{P}(t, P(t), \Xi)\right\} .
$$

Moreover, for every subinterval $[s, t] \subset[0, T]$ the Mosco convergence (2. $\left.\Psi_{3}\right)$ implies

$$
\begin{gathered}
\liminf _{k \rightarrow \infty} \int_{s}^{t}\left(\Psi_{\underline{P}_{\tau_{k}}(r)}^{*}\left(-\widetilde{\Xi}_{\tau_{k}}(r)\right)-\mathfrak{P}\left(r, \widetilde{P}_{\tau_{k}}(r), \widetilde{\Xi}_{\tau_{k}}(r)\right)\right) \mathrm{d} r \\
\geq \int_{s}^{t} \int_{X^{*} \times \mathbb{R}}\left(\Psi_{P(r)}^{*}(-\Xi)-\mathfrak{p}\right) \mathrm{d} \mu_{r}(\Xi, \mathfrak{p}) \mathrm{d} r
\end{gathered}
$$

cf. [MRS13, Thm. A.3]. Finally, by 2.E5 and classical selection techniques there exists a measurable selection $t \mapsto(\Xi(t), \mathfrak{p}(t))$ with

$$
(\Xi(t), \mathfrak{p}(t)) \in \mathcal{S}(t, P(t)) \text { and } \Psi_{P(t)}^{*}(-\Xi(t))-\mathfrak{p}(t)=\min _{(\Xi, p) \in \mathcal{S}(t, P(t))}\left(\Psi_{P(t)}^{*}(-\Xi)-\mathfrak{p}\right)
$$

for all $t \in[0, T]$. From this it is not difficult to deduce that $\Xi \in L^{1}\left(0, T ; X^{*}\right)$. Moreover, for every $[s, t] \subset[0, T]$ we obtain

$$
\begin{aligned}
& \mathscr{E}(t)+\int_{s}^{t} \Psi_{P(r)}(\dot{P}(r)) \mathrm{d} r+\int_{s}^{t}\left(\Psi_{P(r)}^{*}(-\Xi(r))-\mathfrak{P}(r, P(r), \Xi(r))\right) \mathrm{d} r \\
& \stackrel{(1)}{\leq} \mathscr{E}(t)+\int_{s}^{t} \Psi_{P(r)}(\dot{P}(r)) \mathrm{d} r+\int_{s}^{t}\left(\Psi_{P(r)}^{*}(-\Xi(r))-\mathfrak{p}(r)\right) \mathrm{d} r \\
& \stackrel{(2)}{\leq} \mathscr{E}(t)+\int_{s}^{t} \Psi_{P(r)}(\dot{P}(r)) \mathrm{d} r+\int_{s}^{t} \int_{X^{*} \times \mathbb{R}}\left(\Psi_{P(r)}^{*}(-\Xi)-\mathfrak{p}\right) \mathrm{d} \mu_{r}(\Xi, \mathfrak{p}) \mathrm{d} r \\
& \stackrel{(3)}{\leq} \lim _{k \rightarrow \infty} \mathcal{E}\left(\overline{\mathrm{t}}_{\tau_{k}}(t), \bar{P}_{\tau_{k}}(t)\right)+\liminf _{k \rightarrow \infty} \int_{\overline{\mathbf{t}}_{\tau_{k}}(s)}^{\overline{\mathbf{t}}_{\tau_{k}}(t)} \Psi_{\underline{P}_{\tau_{k}}(r)}\left(\dot{P}_{\tau_{k}}(r)\right) \mathrm{d} r \\
& +\liminf _{k \rightarrow \infty} \int_{\overline{\mathrm{t}}_{\tau_{k}}(s)}^{\overline{\mathfrak{\tau}}_{\tau_{k}}(t)}\left(\Psi_{\underline{P}_{\tau_{k}}(r)}^{*}\left(-\widetilde{\Xi}_{\tau_{k}}(r)\right)-\mathfrak{P}\left(r, \widetilde{P}_{\tau_{k}}(r), \widetilde{\Xi}_{\tau_{k}}(r)\right)\right) \mathrm{d} r \\
& \stackrel{(4)}{\leq} \liminf _{k \rightarrow \infty} \mathcal{E}\left(\overline{\mathrm{t}}_{\tau_{k}}(s), \bar{P}_{\tau_{k}}(s)\right) \stackrel{(5)}{=} \mathscr{E}(s),
\end{aligned}
$$

where (1) follows from the definition of the set $\mathcal{S}(\cdot, P(\cdot))$, (2) from the minimality (2.26) of the selection and (3) from the convergences (2.24a $-(2.24 \mathrm{~b})$ combined with $\left(2 . \Psi_{3}\right)$, the energy convergence (2.24d), and the lower semicontinuity estimate (2.25). Finally, (4) follows from passing to the limit $\tau_{k} \downarrow 0$ in the approximate energy-dissipation inequality (2.21), and (5) again from (2.24d).

It remains to show the second assertion in (2.6), namely that

$$
\lim _{\tau_{k} \downarrow 0} \mathcal{E}\left(\overline{\mathrm{t}}_{\tau_{k}}(t), \bar{P}_{\tau_{k}}(t)\right)=\mathcal{E}(t, P(t)) \quad \text { for almost all } t \in(0, T) .
$$

To this aim, we observe that, thanks to estimate (2.23) and the uniform superlinear growth 2. $\Psi_{2}$ of the potentials $\Psi_{P}$, we have $\liminf _{k \rightarrow \infty}\left\|\dot{P}_{\tau_{k}}(t)\right\|_{X}<\infty$ for almost all $t \in(0, T)$. Using now the superlinearity of $\Psi_{P}^{*}$ (cf. (2. $\Psi_{2}$ again) we see that the multivalued maps $\partial \Psi_{P}: X \rightrightarrows X^{*}$ are uniformly bounded on energy sublevels. Therefore 
$\liminf \operatorname{in}_{k \rightarrow \infty}\left\|\bar{\Xi}_{\tau_{k}}(t)\right\|_{X^{*}}<\infty$, where $\bar{\Xi}_{\tau}$ fulfills $\bar{\Xi}_{\tau}(t) \in \mathfrak{F}\left(\overline{\mathrm{t}}_{\tau}(t), \bar{P}_{\tau}(t)\right) \cap\left(-\partial \Psi_{\underline{P}_{\tau}(t)}\left(\dot{P}_{\tau}(t)\right)\right)$ for almost all $t \in(0, T)$, cf. the Euler-Lagrange equation (2.20). Then we can apply the closedness condition (2.. 5 ) and conclude that $\liminf _{k \rightarrow \infty} \mathcal{E}\left(\overline{\mathrm{t}}_{\tau_{k}}(t), \bar{P}_{\tau_{k}}(t)\right)=\mathcal{E}(t, P(t))$ for a.a. $t \in(0, T)$. In view of $(2.24 \mathrm{~d})$, we infer (2.27).

All in all, we have shown that the pair $(P, \Xi)$ satisfies (2.6) and (2.7), and the above chain of inequalities yields the EDI (2.8). This concludes the proof of Theorem 2.7.

\section{Assumptions and main result}

Prior to listing all our assumptions on the energy functionals and on the dissipation potentials involved in the viscoplastic system, we fix some notation and recall some useful identities and inequalities that shall be used throughout.

Notation 3.1 (Matrices). We recall the notation $\mathrm{GL}^{+}(d):=\left\{A \in \mathbb{R}^{d \times d}: \operatorname{det}(A)>0\right\}$. $B y \operatorname{tr}(A)$ we shall denote the trace of a matrix $A \in \mathbb{R}^{d \times d}$, by $\operatorname{cof}(A)$ its cofactor matrix,

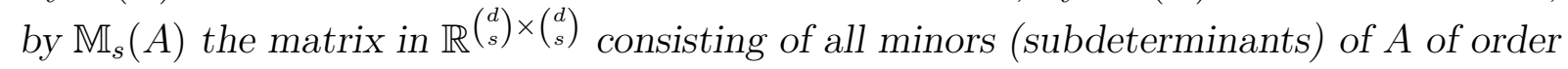
$s$, and by $\mathbb{M}: \mathbb{R}^{d \times d} \rightarrow R^{\mu_{d}}$ the function which maps a matrix to all its minors, with $\mu_{d}:=\sum_{s=1}^{d}\left(\begin{array}{l}d \\ s\end{array}\right)^{2}$. We endow $\mathbb{R}^{d \times d}$ with the inner product

$$
A: B:=\operatorname{tr}\left(A B^{\boldsymbol{\top}}\right)=\sum_{i, j=1}^{d} a_{i j} b_{i j}, \quad \text { giving the Frobenius norm }|A|:=\sqrt{A: A}
$$

Given $r>0$, we shall use the notation

$$
\mathcal{N}_{r}:=\left\{N \in \mathbb{R}^{d \times d}:|N-\mathbf{1}|<r\right\}
$$

Preliminaries: We point out for later use that

$$
A:(B C)=\operatorname{tr}\left(A C^{\top} B^{\top}\right)=\left(A C^{\top}\right): B \quad \text { for all } A, B, C \in \mathbb{R}^{d \times d} .
$$

Furthermore, it follows from definition (3.1) that for every $A, B \in \mathbb{R}^{d \times d}$ we have $|A B| \leq$ $|A||B|$. Combining this with Young's inequality, we deduce for all $A, B \in \mathbb{R}^{d \times d}$ the following estimate

$$
\left|A B^{-1}\right| \geq \frac{|A|}{|B|} \geq C r|A|^{1 / r}-(r-1) C^{r /(r-1)}|B|^{1 /(r-1)} \quad \text { for all } r>1 \text { and all } C>0 .
$$

We will also use that

$$
A^{-1}=\frac{1}{\operatorname{det}(A)} \operatorname{cof}(A)^{\top} \quad \text { for all } A \in \mathrm{GL}^{+}(d) .
$$

and that the cofactor matrix is the Gâteau derivative of the determinant, i.e.

$$
\mathrm{D}_{A}(\operatorname{det}(A))=\operatorname{cof}(A) \quad \text { for all } A \in \mathbb{R}^{d \times d} .
$$


Setup: In what follows, we consider a bounded domain $\Omega \subset \mathbb{R}^{d}$, with Lipschitz boundary $\Gamma$, and denote by $\Gamma_{\text {Dir }}$ the Dirichlet part of the boundary, assumed to have positive surface measure.

In order to highlight the main features of our analysis and avoid overburdening it with technicalities, throughout most of the paper we will impose on $\Gamma_{\text {Dir }}$ time-independent boundary conditions $\varphi_{\text {Dir }}$ for the deformation field; in Section [6 we will outline how our results generalize to time-dependent Dirichlet conditions. Thus, the space for the kinematically admissible deformations is

$$
\mathcal{F}=\left\{\varphi \in W^{1, q_{\Phi}}\left(\Omega ; \mathbb{R}^{d}\right): \varphi=\varphi_{\text {Dir }} \text { on } \Gamma_{\text {Dir }}\right\} \text { for } q_{\Phi}>1 \text { to be specified later. }
$$

The space for the internal variable $P$ is

$$
\mathcal{P}=\left\{P \in W^{1, q_{\mathrm{G}}}\left(\Omega ; \mathbb{R}^{d \times d}\right): P(x) \in \mathrm{GL}^{+}(d) \text { for a.a. } x \in \Omega\right\}
$$

with $q_{\mathrm{G}}>1$ specified later.

Assumptions on the stored energy: The functional $\mathcal{J}:[0, T] \times W^{1, q_{\Phi}}\left(\Omega ; \mathbb{R}^{d}\right) \times$ $L^{p}\left(\Omega ; \mathbb{R}^{d \times d}\right) \rightarrow(-\infty, \infty]$ has the form

$$
\mathcal{J}(t, \varphi, P):=\mathcal{E}_{1}(P)+\mathcal{J}_{2}(t, \varphi, P) \text { for }(t, \varphi, P) \in[0, T] \times W^{1, q_{\Phi}}\left(\Omega ; \mathbb{R}^{d}\right) \times L^{p}\left(\Omega ; \mathbb{R}^{d \times d}\right) .
$$

We now list the conditions on $\mathcal{E}_{1}$ and $\mathcal{J}_{2}$ and postpone to Example 3.4 the discussion of a concrete energy $\mathcal{J}$ complying with all of them.

First of all, $\mathcal{E}_{1}: L^{p}\left(\Omega ; \mathbb{R}^{d \times d}\right) \rightarrow(-\infty, \infty]$ is defined by

$$
\mathcal{E}_{1}(P)= \begin{cases}\int_{\Omega} H(P(x), \nabla P(x)) \mathrm{d} x & \text { if } P \in \mathcal{P} \\ \infty & \text { otherwise }\end{cases}
$$

where the function $H: \mathbb{R}^{d \times d} \times \mathbb{R}^{d \times d \times d} \rightarrow(-\infty, \infty]$ is given by

$$
H(P, A)=K(P)+\frac{1}{q_{\mathrm{G}}}|A|^{q_{\mathrm{G}}} \quad \text { with } q_{\mathrm{G}}>d .
$$

We impose that the hardening function $K: \mathbb{R}^{d \times d} \rightarrow(-\infty, \infty]$ complies with the following conditions:

$K$ is of class $\mathrm{C}^{2}$ in $\mathrm{GL}^{+}(d)$, and

$$
\exists C_{1}, C_{2}>0 \exists q_{\mathrm{P}}, q_{\gamma}>d \forall P \in \mathrm{GL}^{+}(d): K(P) \geq C_{1}\left(|P|^{q_{\mathrm{P}}}+\operatorname{det}(P)^{-q_{\gamma}}\right)-C_{2} \text {. }
$$

The functional $\mathcal{J}_{2}:[0, T] \times W^{1, q_{\Phi}}\left(\Omega ; \mathbb{R}^{d}\right) \times X \rightarrow(-\infty, \infty]$ is defined by

$$
\mathcal{J}_{2}(t, \varphi, P):= \begin{cases}\int_{\Omega} W\left(x, \nabla \varphi(x) P(x)^{-1}\right) \mathrm{d} x-\langle\ell(t), \varphi\rangle_{W^{1, q_{\Phi}}} & \text { if }(\varphi, P) \in \mathcal{F} \times \mathcal{P} \\ \infty & \text { otherwise }\end{cases}
$$

where the external loading $\ell:[0, T] \rightarrow W^{1, q_{\Phi}}\left(\Omega ; \mathbb{R}^{d}\right)^{*}$ fulfills

$$
\ell \in \mathrm{C}^{1}\left([0, T] ; W^{1, q_{\Phi}}\left(\Omega ; \mathbb{R}^{d}\right)^{*}\right)
$$


In most applications the time-dependent loading consists of the work of volume and surface forces, i.e. $\langle\ell(t), \varphi\rangle_{W^{1, q_{\Phi}}}=\int_{\Omega} f(t, x) \cdot \varphi(x) \mathrm{d} x+\int_{\Gamma_{\text {Neu }}} h(t, x) \cdot \varphi(x) \mathrm{d} x$ where $\Gamma_{\text {Neu }}$ is the Neumann part of the boundary $\Gamma=\partial \Omega$.

We require the following conditions on the elastic energy $W: \Omega \times \mathbb{R}^{d \times d} \rightarrow[0, \infty]$ :

$$
\begin{aligned}
& \operatorname{dom}(W)=\Omega \times \mathrm{GL}^{+}(d), \text { i.e. } W(x, F)=\infty \text { for } \operatorname{det} F \leq 0 \text { for all } x \in \Omega, \\
& \exists j \in L^{1}(\Omega) \exists q_{\mathrm{F}}>d \exists C_{3}>0 \forall(x, F) \in \operatorname{dom}(W): W(x, F) \geq j(x)+C_{3}|F|^{q_{\mathrm{F}}}
\end{aligned}
$$

for all $x \in \Omega$ the functional $W(x, \cdot): \mathbb{R}^{d \times d} \rightarrow(-\infty, \infty]$ is polyconvex, i.e. it is a convex function of its minors (cf. Notation 3.1). Namely,

$\exists \mathbb{W}: \Omega \times \mathbb{R}^{\mu_{d}} \rightarrow(-\infty, \infty]$ such that

(i) $\mathbb{W}$ is a normal integrand,

(ii) $\forall(x, F) \in \Omega \times \mathbb{R}^{d \times d}: \quad W(x, F)=\mathbb{W}(x, \mathbb{M}(F))$,

(iii) $\forall x \in \Omega: \mathbb{W}(x, \cdot): \mathbb{R}^{\mu_{d}} \rightarrow(-\infty, \infty]$ is convex,

and $W$ also satisfies

$\exists \delta>0 \quad \exists C_{4}, C_{5}>0 \quad \forall x \in \Omega \forall F \in \mathrm{GL}^{+}(d) \forall N \in \mathcal{N}_{\delta}:$

(i) $W(x, \cdot): \mathrm{GL}^{+}(d) \rightarrow \mathbb{R}$ is differentiable,

(ii) $\left|F^{\top} \mathrm{D}_{F} W(x, F)\right| \leq C_{4}(W(x, F)+1)$, and

(iii) $\left|F^{\top} \mathrm{D}_{F} W(x, F)-(F N)^{\top} \mathrm{D}_{F} W(x, F N)\right| \leq C_{5}|N-\mathbf{1}|(W(x, F)+1)$.

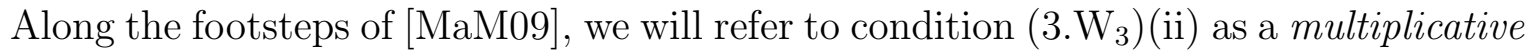
stress control, for it involves the "multiplicative"

$$
\text { Mandel stress tensor } \quad M(F):=F^{\top} \mathrm{D}_{F} W(F) \text {, }
$$

which is estimated, together with its variation (cf. (3.W 1 (iii)), in terms of the energy $W$. This condition goes back to [Bal84, BOP91] and has been recently publicized in [Bal02]. In Section [6, we will see that the treatment of time-dependent Dirichlet boundary conditions requires a multiplicative stress control condition on the Kirchhoff stress tensor $S(F):=$ $\mathrm{D}_{F} W(F) F^{\top}$. Let us also mention that both the stress control conditions $3 . \mathrm{W}_{3}$ and their analogs for the Kirchhoff stress tensor are compatible with polyconvexity and with the physical feasibility requirements that $W(x, F)=\infty$ for $\operatorname{det}(F) \leq 0$, and $W(x, F) \rightarrow \infty$ for $\operatorname{det}(F) \downarrow 0$, cf. Example 3.4 ahead.

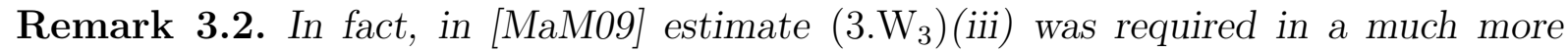
general form, involving on the right-hand side the term $\omega(|N-\mathbf{1}|)$, with the modulus of continuity $\omega:[0, \infty) \rightarrow[0, \infty)$ nondecreasing, and such that $\lim _{\rho \downarrow 0} \omega(\rho)=0$. The discussion in Section 4 (cf. Remark 4.7) will show that in the present context it would be

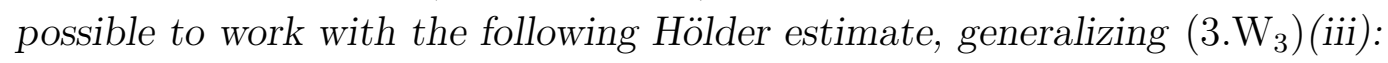

$$
|M(x, F)-M(x, F N)| \leq C|N-\mathbf{1}|^{\alpha_{W}}(W(x, F)+1) \quad \text { for some } \alpha_{W} \in(0,1] .
$$


Assumptions on the dissipation potential: The dissipation potential density $R$ : $\Omega \times \mathbb{R}^{d \times d} \rightarrow[0, \infty)$ is required to satisfy

$$
R: \Omega \times \mathbb{R}^{d \times d} \rightarrow[0, \infty) \text { is a normal integrand, }
$$

for a.a. $x \in \Omega: R(x, \cdot): \mathbb{R}^{d \times d} \rightarrow[0, \infty)$ is convex, with $R(x, 0)=0$, and

$$
R^{*}\left(x, \Xi_{1}\right)=R^{*}\left(x, \Xi_{2}\right) \text { for all } \Xi_{j} \in \partial R(x, V), j=1,2, \text { and } V \in \mathbb{R}^{d \times d} \text {, }
$$

and $R(x, \cdot)$ has a superlinear growth at infinity, uniformly with respect to $x$, i.e.

$$
\begin{aligned}
\exists p \in(1, \infty) \exists C_{R}^{1} C_{R}^{2}, C_{R}^{3}, C_{R}^{4}>0 \quad & \text { for a.a. } x \in \Omega \forall V \in \mathbb{R}^{d \times d}: \\
& C_{R}^{1}|V|^{p}-C_{R}^{2} \leq R(x, V) \leq C_{R}^{3}|V|^{p}+C_{R}^{4} .
\end{aligned}
$$

Notice that in $3 . \mathrm{R}_{2}$ the symbols $R^{*}(x, \cdot)$ denotes the Fenchel-Moreau conjugate of $R(x, \cdot)$, and that $3 . \mathrm{R}_{3}$ implies

$$
\widetilde{C}_{R}^{1}|\Xi|^{p^{\prime}}-\widetilde{C}_{R}^{2} \leq R^{*}(x, \Xi) \leq \widetilde{C}_{R}^{3}|\Xi|^{p^{\prime}}+\widetilde{C}_{R}^{4}
$$

for some $\widetilde{C}_{R}^{j}>0$, where $p^{\prime}=\frac{p}{p-1}$. In Example 3.5 we shall provide a normal integrand $R: \Omega \times \mathbb{R}^{d \times d} \rightarrow[0, \infty)$ complying with $3 . \mathrm{R}_{1}-3 . \mathrm{R}_{3}$.

Setup of the generalized gradient system: energy and dissipation: Starting from the stored energy functional $\mathcal{J}$ and from the dissipation metric $R$, we now introduce the energy functional $\mathcal{E}$ and the (Finsler) family of dissipation potentials $\left(\Psi_{P}\right)_{P \in \mathrm{D}}$ that will allow us to formulate the PDE system (1.8) as the abstract doubly nonlinear evolution equation (2.1) in the state space $X:=L^{p}\left(\Omega ; \mathbb{R}^{d \times d}\right)$.

We consider the reduced energy functional $\mathcal{E}:[0, T] \times X \rightarrow(-\infty, \infty]$ obtained by minimizing out the deformations from $\mathcal{J}$. Recalling $\mathcal{F}$ from (3.7) we set

$$
\mathcal{E}(t, P):=\inf \{\mathcal{J}(t, \varphi, P): \varphi \in \mathcal{F}\}
$$

It is not difficult to check that $\operatorname{dom}(\mathcal{E})$ is of the form $[0, T] \times \mathrm{D}$ for some $\mathrm{D} \subset X$. For this, we note that $\mathcal{E}$ is given by the sum of the functional $\mathcal{E}_{1}$, which is independent of the time-variable, and of a time-dependent, reduced functional $\varepsilon_{2}:[0, T] \times X \rightarrow(-\infty, \infty]$, i.e.

$$
\mathcal{E}(t, P)=\mathcal{E}_{1}(P)+\inf \left\{\mathcal{J}_{2}(t, \varphi, P): \varphi \in \mathcal{F}\right\}=: \mathcal{E}_{1}(P)+\mathcal{E}_{2}(t, P) .
$$

In Lemma 4.2 we shall prove that the functional $\mathcal{E}_{2}$ is bounded from below. Therefore, $\mathcal{E}(t, P)<\infty$ implies $\mathcal{E}_{1}(P)<\infty$. Then, it follows from (3.11), (3.K. inequality that, if $P \in \mathrm{D}$, then $P \in W^{1, q_{\mathrm{G}}}\left(\Omega ; \mathbb{R}^{d \times d}\right)$. Since $q_{\mathrm{G}}>d$, this implies that $P \in \mathrm{C}^{0}\left(\bar{\Omega} ; \mathbb{R}^{d \times d}\right)$, hence $\operatorname{cof}(P) \in \mathrm{C}^{0}\left(\bar{\Omega} ; \mathbb{R}^{d \times d}\right)$. Combining this with formula (3.5) and with the fact that $\operatorname{det}(P)^{-1} \in L^{q_{\gamma}}(\Omega)$ in view of $\left(3 . K_{2}\right)$, we conclude that

$$
\mathrm{D} \subset\left\{P \in \mathcal{P}: P^{-1} \in L^{q_{\gamma}}\left(\Omega ; \mathbb{R}^{d \times d}\right)\right\} .
$$

In fact, we will even prove that any $P \in \mathrm{D}$ satisfies $P^{-1} \in \mathrm{C}^{0}\left(\bar{\Omega} ; \mathbb{R}^{d \times d}\right)$, see (4.12) ahead. 
We are now in the position to introduce the dissipation potentials $\Psi_{P}: X \rightarrow[0, \infty)$, for $P \in \mathrm{D}$. We set

$$
\mathcal{R}(x, P, V):=R\left(x, V P^{-1}\right) \text { for a.a. } x \in \Omega \text {, for all }(P, V) \in \mathrm{GL}^{+}(d) \times \mathbb{R}^{d \times d},
$$

and define

$$
\Psi_{P}(V):=\int_{\Omega} \mathcal{R}(x, P(x), V(x)) \mathrm{d} x \quad \text { for all }(P, V) \in \mathrm{D} \times X .
$$

Notice that the above formula is well-defined thanks to (3.18).

While postponing to Section 4 a thorough discussion of assumptions $\left(3 . \mathrm{K}_{1}\right)-\left(3 . \mathrm{K}_{2}\right)$, $\left(3 . \mathrm{W}_{1}\right)-\left(3 . \mathrm{W}_{3}\right)$, and $\left(3 . \mathrm{R}_{1}-\left(3 . \mathrm{R}_{3}\right)\right.$, let us mention here that they ensure that the energy $\mathcal{E}$ and the dissipation potentials $\left(\Psi_{P}\right)_{P \in \mathrm{D}}$ comply with the abstract assumptions of Theorem 2.7. Thus we will deduce the existence of EDI solutions (in the sense of Definition 2.1) to (2.1).

Statement of the main result: In addition to the assumptions listed above, Theorem 3.3 below requires two further conditions suitably relating the growth exponents involved therein.

Theorem 3.3 (Existence of EDI solutions for viscoplasticity). Let the energy $\mathcal{J}$ and the dissipation potentials $\left(\Psi_{P}\right)_{P \in \mathrm{D}}$ fulfill $\left.3 . \mathrm{K}_{1}\right)-\left(3 . \mathrm{K}_{2}\right)$, (3.L), $\left.3 . \mathrm{W}_{1}\right)-\left(3 . \mathrm{W}_{3}\right)$, and $\left(3 . \mathrm{R}_{1}\right)-$

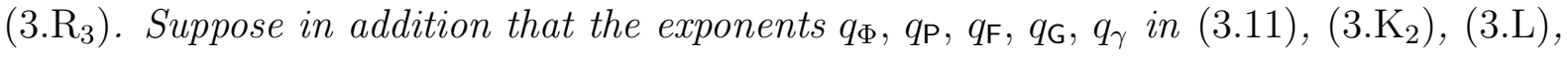
and $3 . \mathrm{W}_{1}$ comply with

$$
\begin{aligned}
& \frac{1}{q_{\Phi}}=\frac{1}{q_{\mathrm{F}}}+\frac{1}{q_{\mathrm{P}}}, \quad q_{\Phi}>d, \\
& \tilde{q}>d, \text { where } \frac{1}{\tilde{q}}:=\frac{2}{q_{\gamma}}+\frac{1}{q_{\mathrm{G}}} .
\end{aligned}
$$

Then, for every initial datum

$$
P_{0} \in \mathcal{P}
$$

there exist an EDI solution $(\varphi, P):[0, T] \rightarrow W^{1, q_{\Phi}}\left(\Omega ; \mathbb{R}^{d}\right) \times W^{1, q_{\mathrm{G}}}\left(\Omega ; \mathbb{R}^{d \times d}\right)$ of the associated generalized gradient system. These solutions satisfy $P \in L^{\infty}\left(0, T ; W^{1, q_{\mathrm{G}}}\left(\Omega ; \mathbb{R}^{d \times d}\right)\right) \cap$ $W^{1, p}\left(0, T ; L^{p}\left(\Omega ; \mathbb{R}^{d \times d}\right)\right)$ and $\varphi \in L^{\infty}\left(0, T ; W^{1, q_{\Phi}}\left(\Omega ; \mathbb{R}^{d}\right)\right)$. Moreover, $\Xi$ given by

$$
\Xi(t, x)=-\Delta_{q_{\mathrm{G}}} P(t, x)+\mathrm{D} K(P(t, x))+B(\nabla \varphi(t, x), P(t, x))
$$

(recalling the notation $\left.B(\nabla \varphi, P)=M\left(\nabla \varphi P^{-1}\right) P^{-\top}\right)$, lies in $L^{p^{\prime}}\left(0, T ; L^{p^{\prime}}\left(\Omega ; \mathbb{R}^{d \times d}\right)\right.$ ) and the triple $(P, \varphi, \Xi)$ satisfies the EDI

$$
\begin{aligned}
\mathcal{J}(t, \varphi(t), P(t))+ & \int_{s}^{t} \int_{\Omega}\left(R\left(x, \dot{P}(r, x) P(r, x)^{-1}\right)+R^{*}\left(x,(-\Xi(r, x)) P(r, x)^{\top}\right)\right) \mathrm{d} x \mathrm{~d} r \\
& \leq \mathcal{J}(s, \varphi(s), P(s))-\int_{s}^{t}\langle\dot{\ell}(r), \varphi(r)\rangle_{W^{1, q_{\Phi}}} \mathrm{d} r
\end{aligned}
$$

for all $t \in(0, T]$, almost all $s \in(0, t)$, and $s=0$.

We conclude by discussing the assumptions $\left(3 . \mathrm{K}_{1}\right)-\left(3 . \mathrm{K}_{2}\right),\left(3 . \mathrm{W}_{1}\right)-\left(3 . \mathrm{W}_{3}\right)$, and $\left(3 . \mathrm{R}_{1}\right)-$ $\left(3 . \mathrm{R}_{3}\right.$ for two concrete examples. 
Example 3.4 (Elastoplastic energy). We consider the hardening function $K: \mathbb{R}^{d \times d} \rightarrow$ $[0, \infty]$

$$
K(P)= \begin{cases}c_{1}|P|^{q_{\mathrm{P}}}+c_{2}\left|\frac{1}{\operatorname{det}(P)}\right|^{q_{\gamma}} & \text { for } P \in \mathrm{GL}^{+}(d) \\ \infty & \text { otherwise }\end{cases}
$$

with $c_{1}, c_{2}>0$ and $q_{\mathrm{P}}, q_{\gamma}>d$. An admissible choice for the elastic stored energy density $W$ (focusing for simplicity on the case of spatially-homogeneous materials) is

$$
W(F)=\left\{\begin{array}{ll}
c_{3}|F|^{q_{\mathrm{F}}}+c_{4}\left|\frac{1}{\operatorname{det}(F)}\right|^{\eta} & \text { for } F \in \mathrm{GL}^{+}(d), \\
\infty & \text { otherwise }
\end{array} \quad \text { with } c_{3}, c_{4}>0 \text { and } q_{\mathrm{F}}>d, \eta>0\right.
$$

In addition, we suppose that the exponents $q_{\mathrm{F}}, q_{\gamma}, q_{\mathrm{P}}$ fulfill (3.21)-(3.22). For instance, we may choose $q_{\Phi}=d+1, q_{\mathrm{F}}=q_{\mathrm{P}}=q_{\mathrm{G}}=2 d+2$, and $q_{\gamma}=4 d+4$.

Following the discussion in [MaM09, Example 3.2], we observe that $W$ complies with $\left.\left(3 . \mathrm{W}_{1}\right)-3 . \mathrm{W}_{2}\right)$. In order to check the Mandel stress control $\left.3 . \mathrm{W}_{3}\right)$, taking into account (3.6) we calculate $\mathrm{D}_{F} W(F)=c_{3} q_{\mathrm{F}}|F|^{q_{\mathrm{F}}-2} F-c_{2} \eta(\operatorname{det}(F))^{-\eta-1} \operatorname{cof}(F)$. Therefore

$$
M(F)=F^{\top} \mathrm{D}_{F} W(F)=c_{3} q_{\mathrm{F}}|F|^{q_{\mathrm{F}}-2} F^{\top} F-c_{2} \eta \operatorname{det}(F)^{-\eta} \underbrace{F^{\mathrm{\top}} \operatorname{det}(F)^{-1} \operatorname{cof}(F)}_{=\mathbf{1} \text { by }(3.5)} .
$$

Using (3.27) and arguing in the same way as in [MaM09, Example 3.2], we conclude $3 . \mathrm{W}_{3}$.

Example 3.5 (Viscoplastic dissipation). Given two measurable functions

$$
\begin{aligned}
& \sigma_{\text {yield }}: \Omega \rightarrow(0, \infty) \text {, such that } \exists \sigma_{*}>0 \text { for a.a. } x \in \Omega: \frac{1}{\sigma_{*}} \leq \sigma(x) \leq \sigma_{*}, \\
& \nu: \Omega \rightarrow(0, \infty) \text {, such that } \exists \nu_{*}>0 \text { for a.a. } x \in \Omega: \nu(x) \geq \nu_{*},
\end{aligned}
$$

we set

$$
R(x, V):=\sigma_{\text {yield }}(x)|V|+\frac{\nu(x)}{2}|V|^{2} .
$$

It can be easily computed that

$$
R^{*}(x, \Xi)=\frac{1}{2 \nu(x)} \operatorname{dist}^{2}(\Xi, \mathrm{E}(x)) \quad \text { for a.a. } x \in \Omega \quad \text { for all } \Xi \in \mathbb{R}^{d \times d},
$$

where $\mathrm{E}(x)$ is the elastic domain $\mathrm{E}(x)=\left\{\Xi \in \mathbb{R}^{d \times d}:|\Xi| \leq \sigma_{\text {yield }}(x)\right\}$, and $\operatorname{dist}(\Xi, \mathrm{E}(x))=$ $\inf _{\Theta \in \mathrm{E}(x)}|\Xi-\Theta|=\min _{\Theta \in \mathrm{E}(x)}|\Xi-\Theta|$. In this case it is immediate to check that (3.R $\mathrm{R}_{1}$ (3.R $\mathrm{R}_{3}$, with $p=2$, are verified.

In engineering one often uses dissipation distances $R(x, V)=\sigma_{\text {yield }}(x)|V|+\frac{\nu(x)}{p}|V|^{p}$ with $p=1+m$ and $0<m \ll 1$, see e.g. [ZR*06, Sec. 4.2] where $m=0.012$ (which means $p=1.012$ and $\left.p^{\prime}=84.33\right)$. Note that in that work the resolved shear stress $\tau_{\alpha}$ already includes the yield stress of the slip system $\alpha$.

\section{Proof of Theorem 3.3}

We will derive Theorem 3.3 as a consequence of the abstract Theorem 2.7, applied in this functional setting: 
1. the state space $X$ is $L^{p}\left(\Omega ; \mathbb{R}^{d \times d}\right)$ with $p$ from $\left(3 . \mathrm{R}_{3}\right)$;

2. the energy functional is $\mathcal{E}$ from (3.16);

3. the dissipation potentials $\left(\Psi_{P}\right)_{P \in \mathrm{D}}$ are given by (3.20).

It will turn out to be particularly effective (cf. the discussion in [MRS13, Sec.3]) to work with a notion of subdifferential for the functional $\mathcal{E}(t, \cdot)$ tailored to the fact that $\mathcal{E}$ arises from a minimization procedure, and that the related set of minimizers is nonempty. Thus, we choose $\mathfrak{F}(t, P)$ at a point $(t, P) \in[0, T] \times \mathrm{D}$ to be the collection of the Gâteau derivatives of $\left\{\mathrm{D}_{P} \mathrm{~J}(t, \varphi, P)\right\}$ with $\varphi$ in

$$
\mathcal{M}(t, P):=\operatorname{Argmin}\{\mathcal{J}(t, \varphi, P): \varphi \in \mathcal{F}\}
$$

(which is nonempty, cf. Lemma 4.2 below). More precisely, we set

4. $\mathfrak{F}:[0, T] \times X \rightrightarrows X^{*}$ is the marginal subdifferential (with respect to the variable $P$ ) of $\mathcal{E}$, defined by

$$
\begin{aligned}
\mathfrak{F}(t, P) & :=\left\{\mathrm{D}_{P} \mathcal{J}(t, \varphi, P): \varphi \in \mathcal{M}(t, P)\right\} \\
& =-\Delta_{q_{\mathrm{G}}} P+\mathrm{D} K(P)+\{B(\cdot, \nabla \varphi, P): \varphi \in \mathcal{M}(t, P)\},
\end{aligned}
$$

(cf. also (4.27) ahead), recalling that $B(\cdot, \nabla \varphi, P)=M\left(\cdot, \nabla \varphi P^{-1}\right) P^{-\top}$ $=\left(\nabla \varphi P^{-1}\right)^{\mathrm{T}} \mathrm{D}_{F} W\left(\cdot, \nabla \varphi P^{-1}\right) P^{-\mathrm{T}}$.

Finally, as suggested by [MRS13] we will work with the following surrogate notion of $\partial_{t} \mathcal{E}$, namely the power functional:

5. $\mathfrak{P}: \operatorname{graph}(\mathfrak{F}) \rightarrow \mathbb{R}$ is defined via

$$
\mathfrak{P}(t, P, \Xi):=\sup _{\varphi \in \mathfrak{R}(t, P, \Xi)} \partial_{t} \mathcal{J}(t, \varphi, P)=\sup _{\varphi \in \Re(t, P, \Xi)}\langle-\dot{\ell}(t), \varphi\rangle_{W^{1, q_{\Phi}}},
$$

where for all $(t, P, \Xi) \in \operatorname{graph}(\mathfrak{F})$ we set

$$
\mathfrak{R}(t, P, \Xi):=\left\{\varphi \in \mathcal{M}(t, P): \Xi=-\Delta_{q_{\mathrm{G}}} P+\mathrm{D} K(P)+M\left(\cdot, \nabla \varphi P^{-1}\right) P^{-\mathrm{T}}\right\} .
$$

By construction $\mathfrak{R}(t, P, \Xi)$ is nonempty on $\operatorname{graph}(\mathfrak{F})$.

In the following series of lemmas we will show that the generalized gradient system $(X, \mathcal{E}, \Psi, \mathfrak{F}, \mathfrak{P})$ complies with assumptions and $\left.2 . \mathrm{E}_{0}-2 . \mathrm{E}_{5}\right)$ and $2 . \Psi_{1}-2 . \Psi_{4}$. In all of the ensuing results, we will specify which of conditions $\left(3 . \mathrm{K}_{1}\right)-\left(3 . \mathrm{K}_{2}\right)$, (3.L), (3.W 1 $\left(3 . \mathrm{W}_{3}\right),\left(3 . \mathrm{R}_{1}\right)-\left(3 . \mathrm{R}_{3}\right)$, and (3.21)-(3.22) actually come into play in the corresponding proofs. For simplicity, from now on we will omit to explicitly indicate the $x$-dependence in $R, W$, and the related quantities. In fact, since we do not use any higher order regularity, it is obvious that the general $x$-dependent case works as well as the homogeneous case. Furthermore, we will use the symbols $c, C$, whose meaning may vary from line to line, for generic positive constants depending on known quantities. 


\subsection{Basic properties of $\mathcal{E}$}

Preliminarily, we discuss the properties of the energy functional $\mathcal{E}_{1}$ from (3.10).

Lemma 4.1 (Coercivity properties of $\left.\mathcal{E}_{1}\right)$. Assume (3.L), $\left.3 . \mathrm{W}_{1}\right)-\left(3 . \mathrm{W}_{2}\right)$, and (3.21)(3.22). Then,

$$
\operatorname{dom}\left(\mathcal{E}_{1}(t, \cdot)\right) \subset\left\{P \in \mathcal{P}: P^{-1} \in W^{1, \tilde{q}}\left(\Omega ; \mathbb{R}^{d \times d}\right)\right\} \quad \text { for every } t \in[0, T],
$$

and

$\forall S>0 \exists R>0 \forall(t, P) \in \operatorname{dom}\left(\varepsilon_{1}\right): \mathcal{E}_{1}(t, P) \leq S \Rightarrow\|P\|_{W^{1, q_{G}}}+\left\|P^{-1}\right\|_{W^{1, \tilde{q}}} \leq R$.

Proof. It follows from (3.11) and $\left.3 . \mathrm{K}_{2}\right)$ that for every $P \in \mathcal{P}$

$$
\mathcal{E}_{1}(t, P) \geq \int_{\Omega} C_{1}\left(|P|^{q_{\mathrm{P}}}+\operatorname{det}(P)^{-q_{\gamma}}\right)+\frac{1}{q_{\mathrm{G}}}|\nabla P|^{q_{\mathrm{G}}} \mathrm{d} x-C .
$$

Therefore, the estimate for $\|P\|_{W^{1, q_{G}}}$ follows from Poincaré's inequality, cf. also the arguments leading to (3.18). In order to prove the bound for $\left\|P^{-1}\right\|_{W^{1, \tilde{q}}}$, we compute

$$
\nabla P^{-1}=-P^{-1} \nabla P P^{-1}
$$

use that $\varepsilon_{1}(t, P) \leq S$ yields an estimate for $\left\|P^{-1}\right\|_{L^{q_{\gamma}}}$ (cf. again (3.18)), and combine it with the bound for $\|\nabla P\|_{L^{q_{G}}}$ via Hölder's inequality, relying on the condition that $\frac{2}{q_{\gamma}}+\frac{1}{q_{\mathrm{G}}}=\frac{1}{\tilde{\tilde{q}}}$ from (3.22). This provides a bound for $\|\nabla P\|_{L^{\tilde{q}}}$. Then, with Poincaré's inequality we obtain the desired estimate for $\left\|P^{-1}\right\|_{W^{1, \tilde{q}}}$, and (4.6) ensues.

We now turn to the reduced energy functional $\varepsilon_{2}$ from (3.17).

Lemma 4.2 (Existence of minimizers for $\left.\mathcal{J}_{2}\right)$. Assume (3.L), $\left.3 . \mathrm{W}_{1}\right)-\left(3 . \mathrm{W}_{2}\right)$, and (3.21)(3.22). Then, $\mathrm{J}_{2}$ defined in (3.12) is coercive, namely

$$
\begin{gathered}
\forall \varrho>0 \exists c_{1}, c_{2}>0 \forall(t, \varphi, P) \in[0, T] \times W^{1, q_{\Phi}}\left(\Omega ; \mathbb{R}^{d}\right) \times L^{p}\left(\Omega ; \mathbb{R}^{d \times d}\right) \text { s.t. } \operatorname{det} P>0 \text { a.e. in } \Omega: \\
\mathcal{J}_{2}(t, \varphi, P) \geq c_{1}\|\varphi\|_{W^{1, q_{\Phi}}}^{q_{\Phi}}-\varrho\|P\|_{L^{q_{\mathrm{P}}}}^{q_{\mathrm{P}}}-c_{2} .
\end{gathered}
$$

Moreover, for all $(t, P) \in[0, T] \times \mathrm{D}$ the set $\mathcal{M}(t, P)$ of minimizers, namely

$$
\mathcal{M}(t, P):=\operatorname{Argmin} \mathcal{J}_{2}(t, \cdot, P)=\left\{\varphi \in \mathcal{F}: \varphi \text { minimizes } \mathcal{J}_{2}(t, \cdot, P) \text { on } \mathcal{F}\right\}
$$

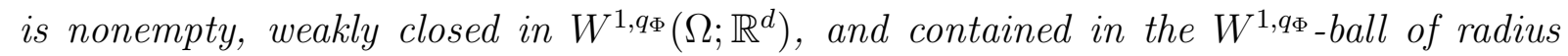
$\hat{r}(P):=c_{3}\left(1+\|P\|_{L^{q_{\mathrm{P}}}}^{q_{\mathrm{P}}}\right)^{1 / q_{\Phi}}$.

Proof. In order to prove coercivity, we use $3 . \mathrm{W}_{1}$ and find

$$
\mathcal{J}_{2}(t, \varphi, P) \geq \int_{\Omega} j(x) \mathrm{d} x+C_{3} \int_{\Omega}\left|\nabla \varphi P^{-1}\right|^{q_{\mathrm{F}}} \mathrm{d} x-\langle\ell(t), \varphi\rangle_{W^{1, q_{\Phi}}} .
$$


Now, we observe that

$$
\begin{aligned}
\int_{\Omega}|\nabla \varphi|^{q_{\Phi}} \mathrm{d} x=\int_{\Omega} \frac{|\nabla \varphi|^{q_{\Phi}}}{|P|^{q_{\Phi}}}|P|^{q_{\Phi}} \mathrm{d} x & \leq\left(\int_{\Omega} \frac{|\nabla \varphi|^{q_{\mathrm{F}}}}{|P|^{q_{\mathrm{F}}}} \mathrm{d} x\right)^{q_{\Phi} / q_{\mathrm{F}}}\left(\int_{\Omega}|P|^{q_{\mathrm{P}}} \mathrm{d} x\right)^{q_{\Phi} / q_{\mathrm{P}}} \\
& \leq\left(\int_{\Omega}\left|\nabla \varphi P^{-1}\right|^{q_{\mathrm{F}}} \mathrm{d} x\right)^{q_{\Phi} / q_{\mathrm{F}}}\left(\int_{\Omega}|P|^{q_{\mathrm{P}}} \mathrm{d} x\right)^{q_{\Phi} / q_{\mathrm{P}}},
\end{aligned}
$$

where the second inequality follows from Hölder's inequality (applied to $(|\nabla \varphi| /|P|)^{q_{\Phi}}$ and $|P|^{q_{\Phi}}$ with exponent $r=q_{\mathrm{F}} / q_{\Phi}$, and the conjugate exponent $r^{\prime}$ is $r^{\prime}=q_{\mathrm{F}} /\left(q_{\mathrm{F}}-q_{\Phi}\right)=q_{\mathrm{P}} / q_{\Phi}$ by virtue of (3.21) $)$, and the third estimate is due to the first of (3.4). Then, for the second term on the right-hand side of (4.10) for every $C>0$ we have

$$
\int_{\Omega}\left|\nabla \varphi P^{-1}\right|^{q_{\mathrm{F}}} \mathrm{d} x \geq \frac{\|\nabla \varphi\|_{L^{q_{\Phi}}}^{q_{\mathrm{F}}}}{\|P\|_{L^{q_{\mathrm{P}}}}^{q_{\mathrm{F}}}} \geq C \frac{q_{\mathrm{F}}}{q_{\Phi}}\|\nabla \varphi\|_{L^{q_{\Phi}}}^{q_{\Phi}}-\frac{q_{\mathrm{F}}}{q_{\mathrm{P}}} C^{q_{\mathrm{P}} / q_{\Phi}}\|P\|_{L^{q_{\mathrm{P}}}}^{q_{\mathrm{P}}}
$$

the latter estimate due to Young's inequality (cf. the second of (3.4)). Then for a given $\rho>0$, we choose the constant $C$ in such a way that $\frac{C_{3}}{2} \frac{q_{\mathrm{F}}}{q_{\mathrm{P}}} C_{\mathrm{P}}^{q_{\mathrm{P}}} / q_{\Phi}=\rho$ and we combine estimate (4.11) with (4.10). Taking into account Korn's and Young's inequalities, we obtain

$$
\begin{aligned}
\mathcal{J}_{2}(t, \varphi, P) & \geq \int_{\Omega} j(x) \mathrm{d} x+C_{\rho}\|\varphi\|_{W^{1, q_{\Phi}}}^{q_{\Phi}}-\rho\|P\|_{L^{q_{\mathrm{P}}}}^{q_{\mathrm{P}}}-\|\ell(t)\|_{W^{1, q_{\Phi}}\left(\Omega ; \mathbb{R}^{d}\right)^{*}}\|\varphi\|_{W^{1, q_{\Phi}}} \\
& \geq \frac{C_{\rho}}{2}\|\varphi\|_{W^{1, q_{\Phi}}}^{q_{\Phi}}-\rho\|P\|_{L^{q_{\mathrm{P}}}}^{q_{\mathrm{P}}}-C^{\prime}\left(1+\|\ell\|_{L^{\infty}\left(0, T ; W^{1, q_{\Phi}}\left(\Omega ; \mathbb{R}^{d}\right)^{*}\right)}^{q_{\Phi^{\prime}}^{\prime}}\right) .
\end{aligned}
$$

Then, (4.8) follows.

In order to prove the existence of minimizers, see (4.9), we preliminarily observe that

$$
\mathrm{D} \subset\left\{P \in \mathcal{P}: P^{-1} \in W^{1, \tilde{q}}\left(\Omega ; \mathbb{R}^{d \times d}\right)\right\} .
$$

This follows from (4.5) and the fact that $\mathcal{J}_{2}$, hence $\mathcal{E}_{2}$, is bounded from below, cf. (4.8). Hence, let us consider an infimizing sequence $\left(\varphi_{k}\right) \subset \mathcal{F}$ for the minimum problem in (3.17). Estimate (4.8) implies that $\left(\varphi_{k}\right)$ is bounded in $W^{1, q_{\Phi}}\left(\Omega ; \mathbb{R}^{d}\right)$, so that there exists $\varphi \in W^{1, q_{\Phi}}\left(\Omega ; \mathbb{R}^{d}\right)$ such that, up to a subsequence, $\varphi_{k} \rightarrow \varphi$ in $W^{1, q_{\Phi}}\left(\Omega ; \mathbb{R}^{d}\right)$. Because of (4.12), we then have $\nabla \varphi_{k} P^{-1} \rightarrow \nabla \varphi P^{-1}$ in $L^{q_{\Phi}}\left(\Omega ; \mathbb{R}^{d \times d}\right)$ for every $P \in \mathrm{D}$, since $P^{-1} \in \mathrm{C}^{0}\left(\bar{\Omega} ; \mathbb{R}^{d \times d}\right)$ in view of (4.12). Hence, the weak continuity of minors of gradients (cf. [Res67, Bal77]) for $q_{\Phi}>d$ and the Cauchy-Binet relations give

$$
\mathbb{M}_{s}\left(\nabla \varphi_{k} P^{-1}\right)=\mathbb{M}_{s}\left(\nabla \varphi_{k}\right) \mathbb{M}_{s}\left(P^{-1}\right) \rightarrow \mathbb{M}_{s}(\nabla \varphi) \mathbb{M}_{s}\left(P^{-1}\right)=\mathbb{M}_{s}\left(\nabla \varphi P^{-1}\right)
$$

in $L^{q_{\Phi} / s}\left(\Omega ; \mathbb{R}^{\left(\begin{array}{l}d \\ s\end{array}\right) \times\left(\begin{array}{l}d \\ s\end{array}\right)}\right)$, for all $s \in\{1, \ldots, d\}$. Ultimately,

$$
\mathbb{M}\left(\nabla \varphi_{k} P^{-1}\right) \rightarrow \mathbb{M}\left(\nabla \varphi P^{-1}\right) \quad \text { in } L^{1}\left(\Omega ; \mathbb{R}^{\mu_{d}}\right)
$$

Then, arguing in the same way as in the proof of [MaM09, Thm. 5.2] to adapt classical lower semicontinuity arguments for Carathéodory integrands (cf. e.g. [Eis79] or [Str90]) to the normal integrand $\mathbb{W}$, we conclude in view of $\left(3 . \mathrm{W}_{2}\right)$ that

$$
\begin{aligned}
\liminf _{k \rightarrow \infty} \int_{\Omega} W\left(\nabla \varphi_{k}(x) P(x)^{-1}\right) \mathrm{d} x & =\liminf _{k \rightarrow \infty} \int_{\Omega} \mathbb{W}\left(\mathbb{M}\left(\nabla \varphi_{k}(x) P(x)^{-1}\right)\right) \mathrm{d} x \\
& \geq \int_{\Omega} \mathbb{W}\left(\mathbb{M}\left(\nabla \varphi(x) P(x)^{-1}\right)\right) \mathrm{d} x
\end{aligned}
$$


Therefore, the direct method in the calculus of variations yields that $\varphi \in \mathcal{F}$ is a minimizer

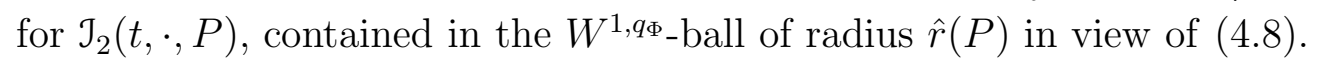

These arguments also show that, up to a subsequence any sequence of minimizers weakly converges in $W^{1, q_{\Phi}}\left(\Omega ; \mathbb{R}^{d}\right)$ to a minimizer. Hence $\mathcal{M}(t, P)$ is weakly sequentially compact in $W^{1, q_{\Phi}}\left(\Omega ; \mathbb{R}^{d}\right)$.

From Lemma 4.2 we now deduce that $\mathcal{E}:[0, T] \times X \rightarrow(-\infty, \infty]$ defined by (3.16) is lower semicontinuous and bounded from below as required by $2 . \mathrm{E}_{0}$ ) (in fact, we will prove that $\mathcal{E}$ is bounded from below by a negative constant but, as previously mentioned, we can always reduce to a positive lower bound by adding a positive constant), and that it complies with conditions (2. $\mathrm{E}_{1}$ and $2 . \mathrm{E}_{3}$.

Lemma 4.3 (Coercivity, lower semicontinuity, and time-dependence of $\mathcal{E}$ ). Assume

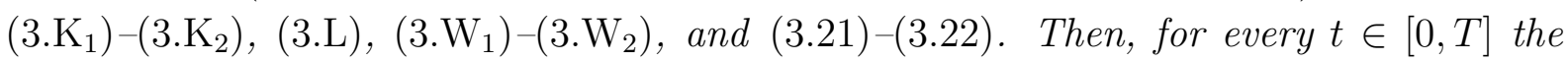
energy $\mathcal{E}(t, \cdot)$ is lower semicontinuous on $X$. Furthermore,

$$
\begin{aligned}
& \exists c_{4}, c_{5}>0 \quad \forall(t, P) \in[0, T] \times \mathrm{D} \forall \varphi \in \mathcal{M}(t, P): \\
& \mathcal{E}(t, P) \geq c_{4}\left(\|\nabla P\|_{L^{q_{G}}}^{q_{\mathrm{G}}}+\|P\|_{L^{q_{\mathrm{P}}}}^{q_{\mathrm{P}}}+\left\|\operatorname{det}(P)^{-1}\right\|_{L^{q_{\gamma}}}^{q_{\gamma}}+\int_{\Omega} W\left(\nabla \varphi P^{-1}\right) \mathrm{d} x\right)-c_{5} .
\end{aligned}
$$

In particular, $\mathcal{E}$ complies with the coercivity $2 . \mathrm{E}_{1}$. It also satisfies

$$
\begin{aligned}
& \forall S>0 \exists R>0 \forall(t, P) \in[0, T] \times \mathrm{D}: \\
& \qquad \mathcal{E}(t, P) \leq S \Rightarrow\|P\|_{W^{1, q_{\mathrm{G}}}}+\left\|P^{-1}\right\|_{W^{1, \tilde{q}}} \leq R .
\end{aligned}
$$

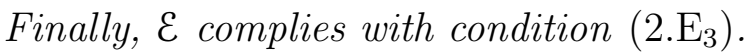

Proof. Estimate (4.15) follows from (3.11) and (3.K. $)$, combined with (4.8) (which clearly yields a lower bound for $\mathcal{E}_{2}$ ), in which for instance we choose $\rho=C_{1} / 4$.

Estimate (4.16) immediately follows from the analogous property (4.6) for $\mathcal{E}_{1}$ and the fact that $\varepsilon_{2}$ is bounded from below thanks to (4.8).

In order to check the lower semicontinuity of $\mathcal{E}(t, \cdot)$, let $P_{n} \rightarrow P$ in $L^{p}\left(\Omega ; \mathbb{R}^{d \times d}\right)$ with $\liminf _{n \rightarrow \infty} \mathcal{E}\left(t, P_{n}\right)<\infty$. Up to a subsequence we have $\sup _{n} \mathcal{E}\left(t, P_{n}\right) \leq C$, hence from (4.16) we deduce that

$$
P_{n} \rightarrow P \text { in } W^{1, q_{\mathrm{G}}}\left(\Omega ; \mathbb{R}^{d \times d}\right) .
$$

Since $q_{\mathrm{G}}>d$ and $\tilde{q}>d$ by (3.22), we have

$$
P_{n} \rightarrow P \quad \text { and } \quad P_{n}^{-1}=\left(\operatorname{det}\left(P_{n}\right)\right)^{-1} \operatorname{cof}\left(P_{n}\right) \rightarrow(\operatorname{det}(P))^{-1} \operatorname{cof}(P)=P^{-1}
$$

in $\mathrm{C}^{0}\left(\bar{\Omega} ; \mathbb{R}^{d \times d}\right)$, so that

$$
P_{n}^{-1} \rightarrow P^{-1} \text { in } W^{1, \tilde{q}}\left(\Omega ; \mathbb{R}^{d \times d}\right) .
$$

Using (4.17) - (4.19) and relying on (3.K. $\mathcal{E}_{1}(P)$. Furthermore, let us choose a sequence $\left(\varphi_{n}\right)_{n}$ with $\varphi_{n} \in \mathcal{M}\left(t, P_{n}\right)$. From $\left\|\varphi_{n}\right\|_{W^{1, q_{\Phi}}} \leq$ $c_{3}\left(1+\left\|P_{n}\right\|_{L^{q_{\mathrm{P}}}}^{q_{\mathrm{P}}}\right)^{1 / q_{\Phi}}$ (cf. Lemma 4.2) , we have (after choosing a not relabeled subsequence) that $\varphi_{n} \rightarrow \widetilde{\varphi}$ in $W^{1, q_{\Phi}}\left(\Omega ; \mathbb{R}^{d}\right)$ for some $\widetilde{\varphi}$. Now, using that $P_{n}^{-1} \rightarrow P^{-1}$ in $\mathrm{C}^{0}\left(\bar{\Omega} ; \mathbb{R}^{d \times d}\right)$ 
by (4.19), we have that $\mathbb{M}_{s}\left(P_{n}^{-1}\right) \rightarrow \mathbb{M}_{s}\left(P^{-1}\right)$ in $\mathrm{C}^{0}$ and thus conclude (cf. (4.13)) that $\mathbb{M}_{s}\left(\nabla \varphi_{n} P_{n}^{-1}\right) \rightarrow \mathbb{M}_{s}\left(\nabla \widetilde{\varphi} P^{-1}\right)$ in $L^{q_{\Phi} / s}\left(\Omega ; \mathbb{R}^{\left(\begin{array}{c}d \\ s\end{array}\right) \times\left(\begin{array}{c}d \\ s\end{array}\right)}\right)$, for all $s \in\{1, \ldots, d\}$. Thus,

$$
\mathcal{E}_{2}(t, P) \leq \mathcal{J}_{2}(t, \widetilde{\varphi}, P) \leq \liminf _{n \rightarrow \infty} \mathcal{J}_{2}\left(t, \varphi_{n}, P_{n}\right)=\liminf _{n \rightarrow \infty} \mathcal{E}_{2}\left(t, P_{n}\right)
$$

Therefore, $\mathcal{E}(t, \cdot)$ is lower semicontinuous.

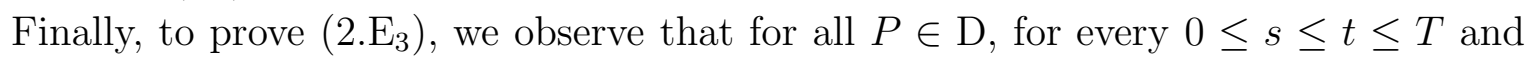
every $\varphi_{s} \in \mathcal{M}(s, P)$ there holds

$$
\begin{aligned}
& \mathcal{E}(t, P)-\mathcal{E}(s, P)=\mathcal{E}_{2}(t, P)-\mathcal{E}_{2}(s, P) \\
& \leq \mathcal{J}_{2}\left(t, \varphi_{s}, P\right)-\mathcal{J}_{2}\left(s, \varphi_{s}, P\right) \\
& =-\left\langle\ell(t)-\ell(s), \varphi_{s}\right\rangle_{W^{1, q_{\Phi}}} \\
& \leq\|\ell(t)-\ell(s)\|_{W^{1, q_{\Phi}}\left(\Omega ; \mathbb{R}^{d}\right)^{*}}\left\|\varphi_{s}\right\|_{W^{1, q_{\Phi}}} \\
& \leq\|\dot{\ell}\|_{L^{\infty}\left(0, T ; W^{\left.1, q_{\Phi}\left(\Omega ; \mathbb{R}^{d}\right)^{*}\right)}\right.}|t-s| c_{3}^{-1 / q_{\Phi}}\left(\mathcal{E}(s, P)+c_{4}\right)^{1 / q_{\Phi}} \\
& \leq C|t-s|(\mathcal{E}(s, P)+1) \text {, }
\end{aligned}
$$

where we have used (3.L) and (4.15) , as well as the trivial inequality $\left(\mathcal{E}(s, P)+c_{4}\right)^{1 / q_{\Phi}} \leq$ $\mathcal{E}(s, P)+c_{4}+1$. Exchanging the roles of $s$ and $t$, from (4.21) and (2.18) we infer $\mid \mathcal{E}(t, P)-$ $\mathcal{E}(s, P)|\leq C| t-s \mid \mathcal{G}(P)$ for every $s, t \in[0, T]$, and $\left[2 . \mathrm{E}_{3}\right)$ follows.

\subsection{Properties of the dissipation potentials}

We now show that the dissipation potentials defined by (3.20) comply with conditions $\left(2 . \Psi_{1}\right)-\left(2 . \Psi_{4}\right)$.

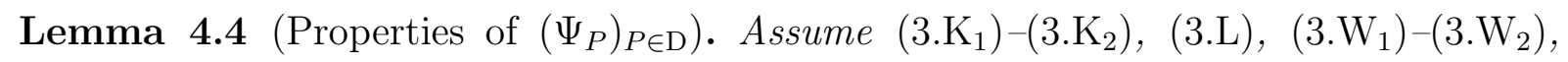

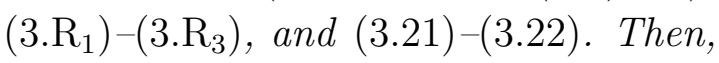

$$
\Psi_{P}^{*}(\Xi)=\int_{\Omega} R^{*}\left(\Xi(x) P(x)^{\top}\right) \mathrm{d} x \quad \text { for every } P \in \mathrm{D} \text { and } \Xi \in X^{*}=L^{p^{\prime}}\left(\Omega ; \mathbb{R}^{d \times d}\right) .
$$

Furthermore, the dissipation potentials $\left(\Psi_{P}\right)_{P \in \mathrm{D}}$ comply with $\left.2 . \Psi_{1}-2 . \Psi_{4}\right)$, and

$$
\begin{gathered}
\forall S>0 \exists c_{6}, c_{7}>0 \forall P \in \mathrm{D} \text { with } \mathcal{G}(P) \leq S \forall V \in X \forall \Xi \in X^{*}: \\
\Psi_{P}(V) \geq c_{6}\|V\|_{L^{p}}^{p}-c_{7} \text { and } \Psi_{P}^{*}(\Xi) \geq c_{6}\|\Xi\|_{L^{p^{\prime}}}^{p^{\prime}}-c_{7} .
\end{gathered}
$$

Proof. Clearly, for every $P \in \mathrm{D}$ the functional $\Psi_{P}$ is well-defined on $L^{p}\left(\Omega ; \mathbb{R}^{d \times d}\right)$; from $\left(3 . \mathrm{R}_{2}\right)$ it follows that $\Psi_{P}$ is convex and lower semicontinuous, and that $\Psi_{P}(0)=0$. Furthermore, using $\left(3 . \mathrm{R}_{3}\right)$, the first estimate in $(\underline{3.4})$, and the fact that $\mathrm{D} \subset \mathrm{C}^{0}\left(\bar{\Omega} ; \mathbb{R}^{d \times d}\right)$, we find for every $(P, V) \in \mathrm{D} \times L^{p}\left(\Omega ; \mathbb{R}^{d \times d}\right)$ the estimate

$$
\Psi_{P}(V) \geq C_{R}^{1} \int_{\Omega}\left|V(x) P(x)^{-1}\right|^{p} \mathrm{~d} x-C_{R}^{2}|\Omega| \geq \frac{C_{R}^{1}}{\|P\|_{L^{\infty}}^{p}} \int_{\Omega}|V(x)|^{p} \mathrm{~d} x-C_{R}^{2}|\Omega|,
$$

which in particular yields that, for $P \in \mathrm{D}$ fixed with $\mathcal{G}(P) \leq S$, the functional $\Psi_{P}$ has uniform superlinear growth on sublevels of $\mathcal{G}$. 
The representation formula (4.22) follows from

$$
\Psi_{P}^{*}(\Xi)=\int_{\Omega} \mathcal{R}^{*}(P(x), \Xi(x)) \mathrm{d} x
$$

(where $\mathcal{R}^{*}$ is the conjugate of $\mathcal{R}$ with respect to the third variable), and the calculation

$$
\begin{aligned}
\mathcal{R}^{*}(P, \Xi)=\sup _{V \in \mathbb{R}^{d \times d}}\left(\Xi: V-R\left(V P^{-1}\right)\right) & =\sup _{W \in \mathbb{R}^{d \times d}}(\Xi: W P-R(W)) \\
& =\sup _{W \in \mathbb{R}^{d \times d}}\left(\Xi P^{\boldsymbol{\top}}: W-R(W)\right)=R^{*}\left(\Xi P^{\boldsymbol{\top}}\right),
\end{aligned}
$$

where the second identity follows from the change of variable $W=V P^{-1}$ and the third one from (3.3). With (4.22) and the second of $\left(3 . \mathrm{R}_{2}\right)$ we find that the functionals $\left(\Psi_{P}^{*}\right)_{P \in \mathrm{D}}$

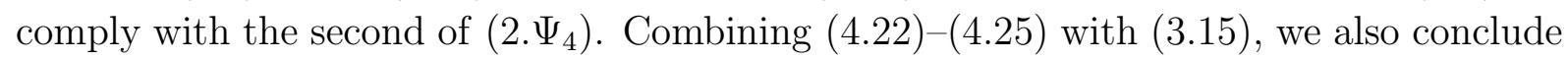
that

$$
\begin{aligned}
\forall(P, \Xi) \in \mathrm{D} \times L^{p^{\prime}}\left(\Omega ; \mathbb{R}^{d \times d}\right): & \\
\Psi_{P}^{*}(\Xi)=\int_{\Omega} R^{*}\left(\Xi(x)\left(P(x)^{-\mathrm{T}}\right)^{-1}\right) \mathrm{d} x & \geq \widetilde{C}_{R}^{1} \int_{\Omega}\left|\Xi(x)\left(P(x)^{-\mathrm{T}}\right)^{-1}\right|^{p^{\prime}}-\widetilde{C}_{R}^{2}|\Omega| \\
& \geq \frac{\widetilde{C}_{R}^{1}}{\left\|P^{-1}\right\|_{L^{\prime}}^{p^{\prime}}} \int_{\Omega}|\Xi(x)|^{p^{\prime}} \mathrm{d} x-\widetilde{C}_{R}^{2}|\Omega| .
\end{aligned}
$$

Then, (2. $\left.\Psi_{2}\right)$ follows from combining (4.24) and (4.26) with estimate (4.16) and the compact embeddings $W^{1, q_{\mathrm{G}}}\left(\Omega ; \mathbb{R}^{d \times d}\right) \subset \mathrm{C}^{0}\left(\bar{\Omega} ; \mathbb{R}^{d \times d}\right)$ and $W^{1, \tilde{q}}\left(\Omega ; \mathbb{R}^{d \times d}\right) \subset \mathrm{C}^{0}\left(\bar{\Omega} ; \mathbb{R}^{d \times d}\right)$.

It remains to prove $\left.2 . \Psi_{3}\right)$. Let $\left(P_{n}\right) \subset \mathrm{D},\left(V_{n}\right) \subset L^{p}\left(\Omega ; \mathbb{R}^{d \times d}\right)$ be sequences as in $\left(2 . \Psi_{3}\right)$. In view of (4.16), we find that $P_{n}^{-1} \rightarrow P^{-1}$ in $\mathrm{C}^{0}\left(\bar{\Omega} ; \mathbb{R}^{d \times d}\right)$, therefore $V_{n} P_{n}^{-1} \rightarrow$ $V P^{-1}$ in $L^{p}\left(\Omega ; \mathbb{R}^{d \times d}\right)$, and the Ioffe theorem [Iof77] ensures that

$\liminf _{n \rightarrow \infty} \Psi_{P_{n}}\left(V_{n}\right)=\liminf _{n \rightarrow \infty} \int_{\Omega} R\left(V_{n}(x) P_{n}(x)^{-1}\right) \mathrm{d} x \geq \liminf _{n \rightarrow \infty} \int_{\Omega} R\left(V(x) P(x)^{-1}\right) \mathrm{d} x=\Psi_{P}(V)$.

Analogously, for $\left(\Xi_{n}\right)_{n}$ with $\Xi_{n} \rightarrow \Xi$ in $X^{*}$ we obtain $\liminf \inf _{n \rightarrow \infty} \Psi_{P_{n}}^{*}\left(\Xi_{n}\right) \geq \Psi_{P}^{*}(\Xi)$.

\subsection{Properties of $\mathfrak{F}$ and $\mathfrak{P}$}

We now turn to the analysis of the marginal subdifferential $\mathfrak{F}$ from (4.2). Recall that its definition involves the Gâteaux derivatives $\mathrm{D}_{P} \mathcal{J}(t, \varphi, P)$ of $\mathcal{J}$, evaluated at minimizers $\varphi \in \mathcal{M}(t, P)$. Observe that, a priori, $\mathrm{D}_{P} \mathrm{~J}(t, \varphi, P)=-\Delta_{q_{\mathrm{G}}} P+\mathrm{D} K(P)+B(\nabla \varphi, P)$ is an element in $W^{1, q_{\mathrm{G}}}(\Omega)^{*}$. In fact, the precise definition of $\mathfrak{F}$ turns out to be

$$
\mathfrak{F}(t, P)=\left\{\Xi \in X^{*}=L^{p^{\prime}}\left(\Omega ; \mathbb{R}^{d \times d}\right): \exists \varphi \in \mathcal{M}(t, P) \text { s.t. } \Xi=\mathrm{D}_{P} \mathcal{J}(, \varphi, \mathcal{P})\right\} .
$$

With Lemma 4.6 below, we show (cf. (4.36) ) that for every $(t, P) \in[0, T] \times \mathrm{D}$ the marginal subdifferential $\mathfrak{F}(t, P)$ contains the Fréchet subdifferential $\partial \mathcal{E}(t, P)$ of $\mathcal{E}$ with respect to the $L^{p}\left(\Omega ; \mathbb{R}^{d \times d}\right)$-topology. We recall that the latter is the multivalued operator $\partial \mathcal{E}$ : $[0, T] \times \mathrm{D} \rightrightarrows L^{p^{\prime}}\left(\Omega ; \mathbb{R}^{d \times d}\right)$ defined at $(t, P) \in[0, T] \times \mathrm{D}$ by

$$
\begin{aligned}
& \Xi \in \partial \mathcal{E}(t, P) \text { if and only if } \\
& \quad \mathcal{E}(t, Q)-\mathcal{E}(t, P) \geq \int_{\Omega} \Xi:(Q-P) \mathrm{d} x+o\left(\|Q-P\|_{L^{p}}\right) \text { as } Q \rightarrow P \text { in } L^{p}\left(\Omega ; \mathbb{R}^{d \times d}\right) .
\end{aligned}
$$


Property (4.36) ahead will be used in Corollary 4.10 to verify the variational sum rule required within the abstract existence theory for gradient systems, cf. (2.E2).

In the proof of Lemma 4.6 and of subsequent results, a key role is played by estimates (4.29)-(4.30), derived next. Note that in the following estimates the variable $P$ is restricted to sets such that $|P|+\left|P^{-1}\right| \leq C_{P}$, whereas $F=\nabla \varphi$ varies in all of $\mathrm{GL}^{+}(d)$.

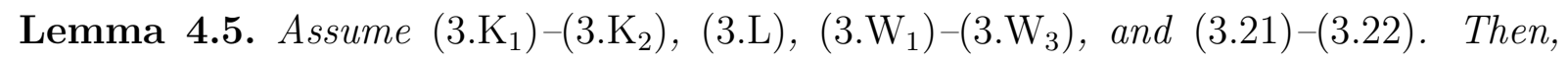
there holds

$$
\begin{aligned}
& \forall C_{P}>0 \exists C_{B}>0 \exists \bar{r}>0 \\
& \forall P \in \mathrm{GL}^{+}(d) \text { with }|P|+\left|P^{-1}\right| \leq C_{P} \forall F \in \mathrm{GL}^{+}(d) \forall N \in \mathcal{N}_{\bar{r}}: \\
& \quad|B(F, N P)-B(F, P)| \leq C_{B}|N-\mathbf{1}|\left(W\left(F P^{-1}\right)+1\right),
\end{aligned}
$$

and

$$
\begin{aligned}
& \forall C_{P}>0 \exists C_{W}>0 \exists \tilde{r}>0 \\
& \forall P_{1}, P_{2} \in \mathrm{GL}^{+}(d) \text { s.t. }\left|P_{1}\right|+\left|P_{1}^{-1}\right| \leq C_{P},\left|P_{1}-P_{2}\right| \leq \tilde{r} \text { and } \forall F \in \mathrm{GL}^{+}(d): \\
& \left|W\left(F P_{1}^{-1}\right)-W\left(F P_{2}^{-1}\right)-B\left(F, P_{1}\right):\left(P_{1}-P_{2}\right)\right| \leq C_{W}\left(W\left(F P_{1}^{-1}\right)+1\right)\left|P_{1}-P_{2}\right|^{2},
\end{aligned}
$$

Proof. Ad (4.29): let us set $P_{N}:=N P$, and observe that $P_{N}^{-1}=P^{-1} N^{-1}$. Furthermore, we have

$$
\left|P_{N}^{-1}-P^{-1}\right| \leq C_{P}\left|N^{-1}-\mathbf{1}\right| \leq 2 C_{P}|N-\mathbf{1}|,
$$

Let us define $\bar{r}:=\min \left\{\left|P^{-1}\right| / 4 C_{P}, \delta\right\}$, with $\delta>0$ from $3 . \mathrm{W}_{3}$. For $N \in \mathcal{N}_{r}$ with $r \leq \bar{r}$, we have that $2 C_{P}|N-\mathbf{1}| \leq\left|P^{-1}\right| / 2$. Therefore we find

$$
\left|P_{N}^{-1}\right| \leq\left|P_{N}^{-1}-P^{-1}\right|+\left|P^{-1}\right| \leq 2\left|P^{-1}\right| \leq 2 C_{P}
$$

Hence

$$
\begin{aligned}
|B(F, N P)-B(F, P)| & \leq\left|M\left(F P_{n}^{-1}\right) P_{N}^{-\mathrm{\top}}-M\left(F P^{-1}\right) P^{-\mathrm{\top}}\right| \\
& \leq\left|\left(M\left(F P_{n}^{-1}\right) P_{N}^{-\mathrm{\top}}-M\left(F P^{-1}\right)\right) P_{N}^{-\mathrm{\top}}\right|+\left|M\left(F P^{-1}\right)\left(P_{N}^{-\mathrm{T}}-P^{-\mathrm{\top}}\right)\right| \\
& \stackrel{(1)}{\leq} C_{5}\left|N^{-1}-\mathbf{1}\right|\left(W\left(F P^{-1}\right)+1\right) 2 C_{P}+C_{4}\left(W\left(F P^{-1}\right)+1\right) C_{P}\left|N^{-1}-\mathbf{1}\right| \\
& \stackrel{(2)}{\leq} 6 C_{P}\left(C_{4}+C 5\right)\left(W\left(F P^{-1}\right)+1\right)|N-\mathbf{1}|,
\end{aligned}
$$

where (1) follows from the multiplicative stress control conditions $\left(3 . \mathrm{W}_{3}\right)$ (ii) and $\left.3 . \mathrm{W}_{3}\right)$ (iii), combined with estimates (4.31) and (4.32), while (2) is due to the last inequality in (4.31). We thus conclude (4.29).

Ad (4.30): By the chain rule we have that

$$
W\left(F P_{1}^{-1}\right)-W\left(F P_{2}^{-1}\right)=\int_{0}^{1} B\left(F, N_{\sigma} P_{1}\right):\left(P_{1}-P_{2}\right) \mathrm{d} \sigma \text { with } N_{\sigma}:=\mathbf{1}+(1-\sigma)\left(P_{2}-P_{1}\right) P_{1}^{-1} .
$$

Clearly, $N_{\sigma} P_{1}=(1-\sigma) P_{2}+\sigma P_{1}$ and

$$
\left|N_{\sigma}-\mathbf{1}\right| \leq(1-\sigma) C_{P}\left|P_{1}-P_{2}\right|
$$


Let us set $\tilde{r}:=\bar{r} / 2 C_{P}$ with $\bar{r}>0$ from (4.29), then for $\left|P_{1}-P_{2}\right| \leq \tilde{r}$ we have that

$$
\left|N_{\sigma}-\mathbf{1}\right| \leq \bar{r} / 2
$$

Therefore,

$$
\begin{aligned}
& \left|W\left(F P_{1}^{-1}\right)-W\left(F P_{2}^{-1}\right)-B\left(F, P_{1}\right):\left(P_{1}-P_{2}\right)\right| \leq \int_{0}^{1}\left|B\left(F, N_{\sigma} P_{1}\right)-B\left(F, P_{1}\right)\right|\left|P_{1}-P_{2}\right| \mathrm{d} \sigma \\
& \stackrel{(1)}{\leq} C_{B}\left(W\left(F P_{1}^{-1}\right)+1\right)\left|P_{1}-P_{2}\right| \int_{0}^{1}\left|N_{\sigma}-\mathbf{1}\right| \mathrm{d} \sigma \stackrel{(2)}{\leq} C_{P} C_{B}\left(W\left(F P_{1}^{-1}\right)+1\right)\left|P_{1}-P_{2}\right|^{2},
\end{aligned}
$$

where (1) is due to the previously proved estimate (4.29), (which applies since $N_{\sigma} \in \mathcal{N}_{\bar{r} / 2}$ by (4.34)), whereas (2) ensues from (4.33). We have thus established (4.30).

We are now in the position to prove Lemma 4.6, where we also derive a bound for the $L^{1}\left(\Omega ; \mathbb{R}^{d \times d}\right)$-norm of the backstress contribution $B$ to $\mathfrak{F}(t, P)$ from the multiplicative stress control condition $\left(3 . \mathrm{W}_{3}\right)(\mathrm{ii})$. This will turn out to be crucial in the proof of the closedness property (2.E5), cf. Lemma 4.9 ahead.

Lemma 4.6 (Marginal subdifferential $\mathfrak{F}$ and Fréchet subdifferential $\partial \mathcal{E}$ ). Assume (3.L), $\left.\left.\left(3 . \mathrm{K}_{1}\right)-3 . \mathrm{K}_{2}\right),\left(3 . \mathrm{W}_{1}\right)-3 . \mathrm{W}_{3}\right)$, and (3.21) -(3.22). Then, the following holds:

1. For every $(t, P) \in[0, T] \times \mathrm{D}$ with $\partial \mathcal{E}(t, P) \neq \emptyset$ we have:

$$
\forall \Xi \in \partial \mathcal{E}(t, P) \forall \varphi \in \mathcal{M}(t, P): \Xi-\mathrm{D} K(P)-B(\nabla \varphi, P)=-\Delta_{q_{\mathrm{G}}} P \text { in } L^{1}\left(\Omega ; \mathbb{R}^{d \times d}\right),
$$

which in particular implies

$$
\partial \mathcal{E}(t, P) \subset \mathfrak{F}(t, P) \subset X^{*} \quad \text { for every }(t, P) \in[0, T] \times \mathrm{D} .
$$

2. For every $(t, P) \in[0, T] \times \mathrm{D}$ and $\varphi \in \mathcal{M}(t, P)$ we have $B(\nabla \varphi, P) \in L^{1}\left(\Omega ; \mathbb{R}^{d \times d}\right)$, and

$$
\exists c_{8}>0 \forall(t, P) \in[0, T] \times \mathrm{D} \forall \varphi \in \mathcal{M}(t, P):\|B(\nabla \varphi, P)\|_{L^{1}} \leq c_{8}\left\|P^{-1}\right\|_{L^{\infty}}(\mathcal{G}(P)+1) .
$$

Proof. Ad (4.35): For the proof of (4.35), we shall use the notation

$$
\bar{\varepsilon}_{1}(P):=\frac{1}{q_{\mathrm{G}}} \int_{\Omega}|\nabla P(x)|^{q_{\mathrm{G}}} \mathrm{d} x .
$$

Since the Fréchet subdifferential of $\bar{\varepsilon}_{1}$ with respect to the $W^{1, q_{\mathrm{G}}}\left(\Omega ; \mathbb{R}^{d \times d}\right)$-topology fulfills $\partial_{W^{1, q_{\mathrm{G}}}} \bar{\varepsilon}_{1}(P)=\left\{-\Delta_{q_{\mathrm{G}}} P\right\} \subset\left(W^{1, q_{\mathrm{G}}}\left(\Omega ; \mathbb{R}^{d \times d}\right)\right)^{*}$, we will establish (4.35) by showing that for all $\Xi \in \partial \mathcal{E}(t, P) \subset X^{*}=L^{p^{\prime}}\left(\Omega ; \mathbb{R}^{d \times d}\right)$ and $\varphi \in \mathcal{M}(t, P)$ we have $\Xi-\mathrm{D} K(P)-B(\nabla \varphi, P) \in$ $\partial_{W^{1, q_{\mathrm{G}}}} \bar{\varepsilon}_{1}(P)$. Then, (4.35) holds, because all terms on the left-hand side lie in $L^{1}\left(\Omega ; \mathbb{R}^{d \times d}\right)$.

Hence, by the definition of Fréchet subdifferentials, we have to prove that

$$
\begin{aligned}
\Lambda\left(\bar{\varepsilon}_{1}\right) & :=\overline{\mathcal{E}}_{1}\left(P_{n}\right)-\bar{\varepsilon}_{1}(P)-\int_{\Omega}(\Xi-\mathrm{D} K(P)-B(\nabla \varphi, P)):\left(P_{n}-P\right) \mathrm{d} x \\
& \geq o\left(\left\|P_{n}-P\right\|_{W^{1, q_{\mathrm{G}}}}\right) \text { as } P_{n} \rightarrow P \text { in } W^{1, q_{\mathrm{G}}}\left(\Omega ; \mathbb{R}^{d \times d}\right) .
\end{aligned}
$$


For this we will in fact exploit that, since $q_{\mathrm{G}}>d$ by (3.21), we also have $P_{n} \rightarrow P$ in $\mathrm{C}^{0}\left(\bar{\Omega} ; \mathbb{R}^{d \times d}\right)$. Now, we observe that

$$
\begin{aligned}
\Lambda\left(\overline{\mathcal{E}}_{1}\right)= & \mathcal{E}\left(t, P_{n}\right)-\mathcal{E}(t, P)-\int_{\Omega} \Xi:\left(P_{n}-P\right) \mathrm{d} x \\
& +\int_{\Omega}\left(K(P)-K\left(P_{n}\right)+\mathrm{D} K(P):\left(P_{n}-P\right)\right) \mathrm{d} x \\
& +\mathcal{E}_{2}(t, P)-\mathcal{E}_{2}\left(t, P_{n}\right)+\int_{\Omega} B(\nabla \varphi, P):\left(P_{n}-P\right) \mathrm{d} x=: \Lambda(\mathcal{E})+\Lambda(K)+\Lambda\left(\mathcal{E}_{2}\right) .
\end{aligned}
$$

In what follows, we will estimate from below the three terms $\Lambda(\cdot)$ individually. First of all, by the definition (4.28) of the Fréchet subdifferential, there holds

$$
\Lambda(\mathcal{E}) \geq o\left(\left\|P_{n}-P\right\|_{L^{p}}\right) \quad \text { as } P_{n} \rightarrow P \text { in } L^{p}\left(\Omega ; \mathbb{R}^{d \times d}\right) .
$$

As for $\Lambda(K)$, we observe that images of the sequence $\left(P_{n}\right)_{n}$ and $P$ belong to a compact subset of $\mathrm{GL}^{+}(d)$. Hence, we have

$$
\begin{aligned}
\Lambda(K) & =\int_{\Omega}\left(K(P)-K\left(P_{n}\right)+\mathrm{D} K(P):\left(P_{n}-P\right)\right) \mathrm{d} x \\
& =\int_{\Omega} \int_{0}^{1}\left(\mathrm{D} K(P)-\mathrm{D} K\left((1-\sigma) P_{n}+\sigma P\right)\right):\left(P_{n}-P\right) \mathrm{d} \sigma \mathrm{d} x \\
& \geq-C\left\|P_{n}-P\right\|_{L^{2}}^{2}=o\left(\left\|P_{n}-P\right\|_{W^{1, q_{G}}}\right)
\end{aligned}
$$

as $P_{n} \rightarrow P$ in $W^{1, q_{\mathrm{G}}}\left(\Omega ; \mathbb{R}^{d \times d}\right)$, where the second estimate follows from the fact that $K$ is of class $\mathrm{C}^{2}$ on $\mathrm{GL}^{+}(d)$. Finally, for $\Lambda\left(\mathcal{E}_{2}\right)$ we choose $\varphi \in \mathcal{M}(t, P)$ and use $\mathcal{E}_{2}\left(t, P_{n}\right) \leq$ $\mathrm{J}_{2}\left(t, \varphi, P_{n}\right)$ to obtain

$$
\begin{aligned}
\Lambda\left(\mathcal{E}_{2}\right) & =\mathcal{E}_{2}(t, P)-\mathcal{E}_{2}\left(t, P_{n}\right)+\int_{\Omega} B(\nabla \varphi, P):\left(P_{n}-P\right) \mathrm{d} x \\
& \geq \int_{\Omega} W\left(\nabla \varphi P^{-1}\right)-W\left(\nabla \varphi P_{n}^{-1}\right)+B(\nabla \varphi, P):\left(P_{n}-P\right) \mathrm{d} x \\
& \stackrel{(1)}{\geq}-C_{W} \int_{\Omega}\left(W\left(\nabla \varphi P^{-1}\right)+1\right)\left|P_{n}-P\right|^{2} \mathrm{~d} x \\
& \stackrel{(2)}{\geq}-C(\mathcal{E}(t, P)+1)\left\|P_{n}-P\right\|_{L^{\infty}}^{2} \stackrel{(3)}{\geq}-C(\mathcal{G}(P)+1)\left\|P_{n}-P\right\|_{W^{1, q_{G}}}^{2} .
\end{aligned}
$$

Here, (1) follows from estimate (4.30), which applies since $P \in \mathrm{D}$ and we may suppose that $\left\|P_{n}-P\right\|_{L^{\infty}} \leq \tilde{r}$, with $\tilde{r}$ from (4.30), as $P_{n} \rightarrow P$ in $\mathrm{C}^{0}\left(\bar{\Omega} ; \mathbb{R}^{d \times d}\right)$. Then, estimate (2) ensues from (4.15), and (3) from (2.18). All in all, we infer that $\Lambda\left(\mathcal{E}_{2}\right) \geq o\left(\left\|P_{n}-P\right\|_{W^{1, q_{\mathrm{G}}}}\right)$ as $P_{n} \rightarrow P$ in $W^{1, q_{\mathrm{G}}}\left(\Omega ; \mathbb{R}^{d \times d}\right)$. This gives (4.38), hence (4.35).

Ad (4.37): In view of (3.W $)$ (ii), there holds

$$
\begin{aligned}
\int_{\Omega}|B(\nabla \varphi(x), P(x))| \mathrm{d} x & \leq C_{4} \int_{\Omega}\left(W\left(\nabla \varphi(x) P(x)^{-1}\right)+1\right)\left|P(x)^{-\mathrm{T}}\right| \mathrm{d} x \\
& \leq C_{4}\left\|P^{-1}\right\|_{L^{\infty}}\left(c_{3}^{-1} \mathcal{E}(t, P)+|\Omega|\right)
\end{aligned}
$$

where the last inequality ensues from (4.15). Hence, (4.37) is established. This concludes the proof. 


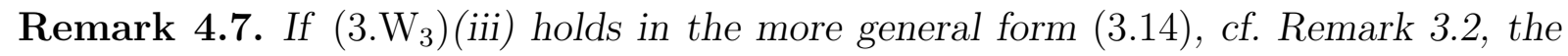
proof of (4.35) still goes through. Indeed, in this case we would have

$$
\begin{aligned}
\Lambda\left(\varepsilon_{2}\right) & \geq-C_{W} \int_{\Omega}\left(W\left(\nabla \varphi P^{-1}\right)+1\right)\left|P_{n}-P\right|^{1+\alpha_{W}} \mathrm{~d} x \\
& \geq-C(\mathcal{G}(P)+1)\left\|P_{n}-P\right\|_{W^{1, q_{G}}}^{1+\alpha_{W}}=o\left(\left\|P_{n}-P\right\|_{W^{1, q_{G}}}\right) .
\end{aligned}
$$

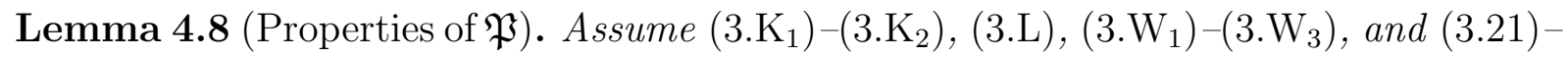
(3.22). Then,

$$
\mathfrak{P}(t, P, \Xi):=\sup _{\varphi \in \mathfrak{R}(t, P, \Xi)}\langle-\dot{\ell}(t), \varphi\rangle_{W^{1, q_{\Phi}}}=\max _{\varphi \in \mathfrak{R}(t, P, \Xi)}\langle-\dot{\ell}(t), \varphi\rangle_{W^{1, q_{\Phi}}}
$$

and the function $\mathfrak{P}: \operatorname{graph}(\mathfrak{F}) \rightarrow \mathbb{R}$ complies with (2.E $)$.

Proof. We first observe that for every $(t, P, \Xi) \in \operatorname{graph}(\mathfrak{F})$ the set

$\mathfrak{R}(t, P, \Xi)$ is nonempty and weakly sequentially compact in $W^{1, q_{\Phi}}\left(\Omega ; \mathbb{R}^{d}\right)$.

Indeed, every sequence $\left(\varphi_{n}\right)_{n} \subset \mathfrak{R}(t, P, \Xi)$ is bounded in $W^{1, q_{\Phi}}\left(\Omega ; \mathbb{R}^{d}\right)$ thanks to (4.15), hence up to a subsequence it weakly converges to some $\varphi$. From the arguments in the proof of Lemma 4.9 ahead (cf. Step 5), it will follow that $\varphi \in \mathfrak{R}(t, P, \Xi)$. Thus, it is immediate to see that the sup in formula (4.41) is indeed a max.

Furthermore, the function $\mathfrak{P}: \operatorname{graph}(\mathfrak{F}) \rightarrow \mathbb{R}$ defined by (4.3) is a Borel function because it is the sup of Borel functions.

For the lower estimate of $\mathfrak{P}$ in $\left(2 . \mathrm{E}_{4}\right)$, we consider first $(t, P) \in[0, T] \times \mathrm{D}, h \in(0, T-t]$ and $\varphi(t) \in \mathcal{M}(t, P)$ to obtain

$$
\frac{\mathcal{E}(t+h, P)-\mathcal{E}(t, P)}{h}=\frac{\mathcal{E}_{2}(t+h, P)-\mathcal{E}_{2}(t, P)}{h} \leq \frac{1}{h}\langle-\ell(t+h)+\ell(t), \varphi(t)\rangle_{W^{1, q_{\Phi}}},
$$

whence $\lim \sup _{h \downarrow 0} \frac{\varepsilon(t+h, P)-\varepsilon(t, P)}{h} \leq \mathfrak{P}(t, P, \Xi)$. In order to prove the upper estimate $\mathfrak{P}(t, P, \Xi) \leq \lim _{h \downarrow 0} \frac{\varepsilon(t, P)-\varepsilon(t-h, P)}{h}$, it is sufficient to observe that

$$
\frac{\mathcal{E}(t+h, P)-\mathcal{E}(t, P)}{h} \geq \frac{1}{h}\langle\ell(t+h)-\ell(t), \varphi(t)\rangle_{W^{1, q_{\Phi}}},
$$

for every $\varphi(t) \in \mathcal{M}(t, P)$, and then take the limit as $h \downarrow 0$. On the other hand, it follows from (3.L) and (4.15) that

$$
\begin{aligned}
|\mathfrak{P}(t, P, \Xi)| \leq\|\dot{\ell}(t)\|_{\left(W^{\left.1, q_{\Phi}\right)^{*}}\right.} \cdot \sup _{\varphi \in \mathcal{M}(t, P)}\|\varphi\|_{W^{1, q_{\Phi}}} & \leq c_{3}\|\dot{\ell}(t)\|_{\left(W^{\left.1, q_{\Phi}\right)^{*}}\right.}\left(1+\|P\|_{L^{q_{\mathrm{P}}}}^{q_{\mathrm{P}}}\right)^{1 / q_{\Phi}} \\
& \leq C(\mathcal{G}(P)+1)^{1 / q_{\Phi}} \leq \widetilde{C}(\mathcal{G}(P)+1) .
\end{aligned}
$$

Therefore, 2.E 2 is fulfilled.

We will now show that the triple $(\mathcal{E}, \mathfrak{F}, \mathfrak{P})$ complies with a more general form of the closedness condition (2. $\left.\mathrm{E}_{5}\right)$, where a sequence $\left(t_{n}\right)_{n}$ is also considered. This is the most difficult step, which involves the ideas developed in [DFT05] and, more abstractly, in FrM06, KZM10]. 
Lemma 4.9 (Closedness for $(\mathcal{E}, \mathfrak{F}, \mathfrak{P}))$. Assume $\left(3 . \mathrm{K}_{1}\right)-\left(3 . \mathrm{K}_{2}\right)$, (3.L), $3 . \mathrm{W}_{1}-\left(3 . \mathrm{W}_{3}\right)$, and (3.21)-(3.22). Then, 2.E holds.

Proof. Let $\left(t_{n}\right)_{n} \subset[0, T],\left(P_{n}\right)_{n} \subset L^{p}\left(\Omega ; \mathbb{R}^{d \times d}\right)$, and $\left(\Xi_{n}\right)_{n} \subset L^{p^{\prime}}\left(\Omega ; \mathbb{R}^{d \times d}\right)$ with $\Xi_{n} \in$ $\mathfrak{F}\left(t_{n}, P_{n}\right)$ for all $n \in \mathbb{N}$ fulfill as $n \rightarrow \infty$

$$
\begin{aligned}
& t_{n} \rightarrow t, \quad P_{n} \rightarrow P \text { in } L^{p}\left(\Omega ; \mathbb{R}^{d \times d}\right), \Xi_{n} \rightarrow \Xi \text { in } L^{p^{\prime}}\left(\Omega ; \mathbb{R}^{d \times d}\right), \\
& \mathcal{E}\left(t_{n}, P_{n}\right) \rightarrow \mathscr{E} \text { and } \mathfrak{P}\left(t_{n}, P_{n}, \Xi_{n}\right) \rightarrow \mathscr{P} \text { in } \mathbb{R} .
\end{aligned}
$$

We will split the proof that

$$
\Xi \in \mathfrak{F}(t, P), \quad \mathscr{E}=\mathcal{E}(t, P), \quad \mathscr{P} \leq \mathfrak{P}(t, P, \Xi)
$$

in several steps.

Step 1: lower semicontinuity inequality for the energies. From $\sup _{n} \mathcal{E}\left(t_{n}, P_{n}\right)<$ $\infty$ and from (4.16) we infer convergences (4.17) -(4.19) for $\left(P_{n}\right)_{n}$ and $\left(P_{n}^{-1}\right)_{n}$. Thus we have $\liminf _{n \rightarrow \infty} \mathcal{E}_{1}\left(P_{n}\right) \geq \mathcal{E}_{1}(P)$ as well as, with the very same argument as in the proof of Lemma 4.3, $\lim \inf _{n \rightarrow \infty} \varepsilon_{2}\left(t_{n}, P_{n}\right) \geq \varepsilon_{2}(t, P)$.

Step 2: convergence of the minimizers. Take any $\left(\varphi_{n}\right)_{n}$ with $\varphi_{n} \in \mathcal{M}\left(t_{n}, P_{n}\right)$ for every $n \in \mathbb{N}$. As we have seen in the proof of Lemma 4.3, up to a subsequence $\varphi_{n} \rightarrow \varphi$ for some $\varphi \in \mathcal{F}$. From $\varphi_{n} \in \operatorname{Argmin}_{\varphi \in \mathcal{F}} \mathcal{J}_{2}\left(t_{n}, \varphi_{n}, P_{n}\right)$ we deduce

$$
\int_{\Omega} W\left(\nabla \varphi_{n} P_{n}^{-1}\right) \mathrm{d} x-\left\langle\ell\left(t_{n}\right), \varphi_{n}\right\rangle_{W^{1, q_{\Phi}}} \leq \int_{\Omega} W\left(\nabla \eta P_{n}^{-1}\right) \mathrm{d} x-\left\langle\ell\left(t_{n}\right), \eta\right\rangle_{W^{1, q_{\Phi}}}
$$

for all $\eta \in \mathcal{F}$. Moreover, using (4.18) we see that $\int_{\Omega} W\left(\nabla \eta P_{n}{ }^{-1}\right) \mathrm{d} x-\left\langle\ell\left(t_{n}\right), \eta\right\rangle_{W^{1, q_{\Phi}}} \rightarrow$ $\int_{\Omega} W\left(\nabla \eta P^{-1}\right) \mathrm{d} x-\langle\ell(t), \eta\rangle_{W^{1, q_{\Phi}}}$ for all $\eta \in \mathcal{F}$, and combining (4.20) with (4.45), we conclude that $\varphi \in \mathcal{M}(t, P)$. Moreover, choosing $\eta=\varphi$ in (4.45), we find

$$
\begin{aligned}
& \limsup _{n \rightarrow \infty}\left(\int_{\Omega} W\left(\nabla \varphi_{n} P_{n}^{-1}\right) \mathrm{d} x-\left\langle\ell\left(t_{n}\right), \varphi_{n}\right\rangle_{W^{1, q_{\Phi}}}\right) \\
& \leq \limsup _{n \rightarrow \infty}\left(\int_{\Omega} W\left(\nabla \varphi P_{n}^{-1}\right) \mathrm{d} x-\left\langle\ell\left(t_{n}\right), \varphi\right\rangle_{W^{1, q_{\Phi}}}\right) \\
& =\int_{\Omega} W\left(\nabla \varphi P^{-1}\right) \mathrm{d} x-\langle\ell(t), \varphi\rangle_{W^{1, q_{\Phi}}}=\mathcal{E}_{2}(t, P) .
\end{aligned}
$$

Combining this with (4.20), we ultimately have $\varepsilon_{2}\left(t_{n}, P_{n}\right) \rightarrow \mathcal{E}_{2}(t, P)$ as $n \rightarrow \infty$, whence

$$
\lim _{n \rightarrow \infty} \int_{\Omega} W\left(\nabla \varphi_{n} P_{n}^{-1}\right) \mathrm{d} x=\int_{\Omega} W\left(\nabla \varphi P^{-1}\right) \mathrm{d} x .
$$

Step 3: further compactness arguments and convergence of the energies. In view of (4.2), the sequence $\Xi_{n} \in \mathfrak{F}\left(t_{n}, P_{n}\right)$ in (4.44) is given for every $n \in \mathbb{N}$ by

$$
\Xi_{n}=-\Delta_{q_{\mathrm{G}}} P_{n}+\mathrm{D} K\left(P_{n}\right)+B\left(\nabla \varphi_{n}, P_{n}\right) \quad \text { for some } \varphi_{n} \in \mathcal{M}\left(t_{n}, P_{n}\right)
$$


Estimate (4.37), $\sup _{n} \mathcal{E}\left(t_{n}, P_{n}\right)<\infty$, (4.16), and $\tilde{q}>d$ yields $\sup _{n}\left\|B\left(\nabla \varphi_{n}, P_{n}\right)\right\|_{L^{1}} \leq C$. Moreover, unsing $K \in \mathrm{C}^{1}\left(\mathrm{GL}^{+}(d) ; \mathbb{R}\right)$ and (4.18) we deduce $\sup _{n}\left\|\mathrm{D} K\left(P_{n}\right)\right\|_{L^{\infty}} \leq C$. By comparison in (4.47) we infer that

$$
\sup _{n \in \mathbb{N}}\left\|-\Delta_{q_{\mathrm{G}}} P_{n}\right\|_{L^{1}} \leq C
$$

Since $q_{\mathrm{G}}>d$, we have $L^{1}\left(\Omega ; \mathbb{R}^{d \times d}\right) \Subset W^{1, q_{\mathrm{G}}}\left(\Omega ; \mathbb{R}^{d \times d}\right)^{*}$, such that, up to a (not relabeled) subsequence, $-\Delta_{q_{\mathrm{G}}} P_{n} \rightarrow \chi$ in $W^{1, q_{\mathrm{G}}}\left(\Omega ; \mathbb{R}^{d \times d}\right)^{*}$ for some $\chi$. Combining this with (4.17) and taking into account that the monotone map $P \mapsto-\Delta_{q_{\mathrm{G}}} P$ has a weakly-strongly closed graph in $W^{1, q_{\mathrm{G}}}\left(\Omega ; \mathbb{R}^{d \times d}\right) \times W^{1, q_{\mathrm{G}}}\left(\Omega ; \mathbb{R}^{d \times d}\right)^{*}$, we ultimately conclude that $\chi=-\Delta_{q_{\mathrm{G}}} P$, and that along the same sequence as in (4.44) there holds

$$
-\Delta_{q_{\mathrm{G}}} P_{n} \rightarrow-\Delta_{q_{\mathrm{G}}} P \text { in } W^{1, q_{\mathrm{G}}}\left(\Omega ; \mathbb{R}^{d \times d}\right)^{*} .
$$

Thus, the assumed weak convergence $P_{n} \rightarrow P$ in $W^{1, q_{\mathrm{G}}}\left(\Omega ; \mathbb{R}^{d \times d}\right)$, cf. (4.17), improves to

$$
P_{n} \rightarrow P \text { in } W^{1, q_{\mathrm{G}}}\left(\Omega ; \mathbb{R}^{d \times d}\right) .
$$

Now, taking into account that $K$ is of class $\mathrm{C}^{0}$ on $\mathrm{GL}^{+}(d)$ we conclude that

$$
\int_{\Omega} K\left(P_{n}\right) \mathrm{d} x \rightarrow \int_{\Omega} K(P) \mathrm{d} x \quad \text { as } n \rightarrow \infty .
$$

Combining this with (4.50) and recalling (3.10)-(3.11), we have $\mathcal{E}_{1}\left(P_{n}\right) \rightarrow \mathcal{E}_{1}(P)$ so that, by (4.46) we have

$$
\mathcal{E}\left(t_{n}, P_{n}\right) \rightarrow \mathcal{E}(t, P) \text { as } n \rightarrow \infty,
$$

whence the second of (4.44).

Step 4: convergence of the stresses. With (4.18) and the $\mathrm{C}^{0}$-regularity of $\mathrm{D} K$ we infer

$$
\mathrm{D} K\left(P_{n}\right) \rightarrow \mathrm{D} K(P) \text { in } L^{\infty}\left(\Omega ; \mathbb{R}^{d \times d}\right) .
$$

We now show that

$$
\begin{aligned}
& B\left(\nabla \varphi_{n}, P_{n}\right) \rightarrow B(\nabla \varphi, P) \text { in } L^{1}\left(\Omega ; \mathbb{R}^{d \times d}\right), \text { viz. } \\
& \int_{\Omega} B\left(\nabla \varphi_{n}, P_{n}\right): Q \mathrm{~d} x \rightarrow \int_{\Omega} B(\nabla \varphi, P): Q \mathrm{~d} x
\end{aligned}
$$

for all $Q \in L^{\infty}\left(\Omega ; \mathbb{R}^{d \times d}\right)$, where $\varphi_{n} \in \mathcal{M}\left(t_{n}, P_{n}\right)$ and $\varphi \in \mathcal{M}(t, P)$ are from the previous steps. To this aim, we mimic the proof of [FrM06, Prop. 3.3]. We fix $Q \in L^{\infty}\left(\Omega ; \mathbb{R}^{d \times d}\right)$ and $h>0$. On the one hand, we have

$$
\begin{aligned}
& \left|\frac{1}{h} \int_{\Omega}\left(W\left(\nabla \varphi_{n}\left(P_{n}+h Q\right)^{-1}\right)-W\left(\nabla \varphi_{n} P_{n}^{-1}\right)-h B\left(\nabla \varphi_{n}, P_{n}\right): Q\right) \mathrm{d} x\right| \\
& \stackrel{(1)}{\leq} \frac{1}{h} C_{W} \int_{\Omega}\left(W\left(\nabla \varphi_{n} P_{n}{ }^{-1}\right)+1\right)|h Q|^{2} \mathrm{~d} x \stackrel{(2)}{\leq} h C\left(\mathcal{G}\left(P_{n}\right)+1\right)\|Q\|_{L^{\infty}}^{2}=: \omega(h),
\end{aligned}
$$

where (1) follows from applying estimate (4.30) with the choices $P_{1}:=P_{n}$ and $P_{2}:=$ $P_{n}+h Q$. Note that $\left\|P_{n}\right\|_{L^{\infty}}+\left\|P_{n}^{-1}\right\|_{L^{\infty}} \leq C$ thanks to the energy bound $\sup _{n} \mathcal{E}\left(t_{n}, P_{n}\right)<$ 
$\infty$, combined with the coercivity property (4.16) and the continuous embeddings $W^{1, q_{\gamma}}\left(\Omega ; \mathbb{R}^{d \times d}\right) \subset L^{\infty}\left(\Omega ; \mathbb{R}^{d \times d}\right)$ and $W^{1, \tilde{q}}\left(\Omega ; \mathbb{R}^{d \times d}\right) \subset L^{\infty}\left(\Omega ; \mathbb{R}^{d \times d}\right)$. We may then take $h$ sufficiently small, in such a way that $\left\|P_{1}-P_{2}\right\|_{L^{\infty}}=\left\|P_{n}-\left(P_{n}+h Q\right)\right\|_{L^{\infty}}=h\|Q\|_{L^{\infty}} \leq \tilde{r}$. Then, (2) ensues from very same calculations as in (4.40). Hence, $\omega(h) \rightarrow 0$ as $h \rightarrow 0$. On the other hand, the polyconvexity of $W$ combined with the very same arguments as in the proof of Lemma 4.2, and the second of (4.46) yield

$$
\begin{aligned}
& \liminf _{n \rightarrow \infty} \int_{\Omega}\left(W\left(\nabla \varphi_{n}\left(P_{n}+h Q\right)^{-1}\right)-W\left(\nabla \varphi_{n} P_{n}^{-1}\right)\right) \mathrm{d} x \\
& \geq \frac{1}{h} \int_{\Omega}\left(W\left(\nabla \varphi(P+h Q)^{-1}\right)-W\left(\nabla \varphi P^{-1}\right)\right) \mathrm{d} x .
\end{aligned}
$$

Estimate (4.55) and the above inequality yield, for all $h>0$, the estimate

$$
\begin{aligned}
& \liminf _{n \rightarrow \infty} \int_{\Omega} B\left(\nabla \varphi_{n}, P_{n}\right): Q \mathrm{~d} x \\
& \quad \geq \limsup _{n \rightarrow \infty} \frac{1}{h} \int_{\Omega}\left(W\left(\nabla \varphi_{n}\left(P_{n}+h Q\right)^{-1}\right)-W\left(\nabla \varphi_{n} P_{n}^{-1}\right)\right) \mathrm{d} x-\omega(h) \\
& \quad \geq \frac{1}{h} \int_{\Omega} B i g\left(W\left(\nabla \varphi(P+h Q)^{-1}\right)-W\left(\nabla \varphi P^{-1}\right)\right) \mathrm{d} x-\omega(h) \\
& \quad \geq \int_{\Omega} B(\nabla \varphi, P): Q \mathrm{~d} x-2 \omega(h) .
\end{aligned}
$$

where the last inequality follows from (4.55) written for $P$ instead of $P_{n}$. Exchanging $Q$ with $-Q$, we analogously infer that

$$
\limsup _{n \rightarrow \infty} \int_{\Omega} B\left(\nabla \varphi_{n}, P_{n}\right): Q \mathrm{~d} x \leq \int_{\Omega} B(\nabla \varphi, P): Q \mathrm{~d} x+2 \omega(h) .
$$

We conclude (4.54) taking the limit of (4.56) and (4.57) as $h \rightarrow 0$.

All in all, (4.49), (4.53), and (4.54) imply that the sequence $\Xi_{n}$ in (4.44) weakly converges in $L^{p^{\prime}}\left(\Omega ; \mathbb{R}^{d \times d}\right)$ to $\Xi=-\Delta_{q_{\mathrm{G}}} P+\mathrm{D} K(P)+B(\nabla \varphi, P)$, which belongs to $\mathfrak{F}(t, P)$. This proves the first of (4.44).

Step 5: Upper semicontinuity of the powers. The sequence $\left(\mathfrak{P}\left(t_{n}, P_{n}, \Xi_{n}\right)\right)_{n}$ from (4.43) is given for every $n \in \mathbb{N}$ by $\mathfrak{P}\left(t_{n}, P_{n}, \Xi_{n}\right)=-\left\langle\dot{\ell}\left(t_{n}\right), \tilde{\varphi}_{n}\right\rangle_{W^{1, q_{\Phi}}}$ for some $\tilde{\varphi}_{n} \in$ $\mathfrak{R}\left(t_{n}, P_{n}, \Xi_{n}\right)$, which thus fulfills $\Xi_{n}=-\Delta_{q_{\mathrm{G}}} P_{n}+\mathrm{D} K\left(P_{n}\right)+B\left(\nabla \tilde{\varphi}_{n}, P_{n}\right)$. The arguments from Steps 2 and 4 yield that, up to a further (not relabeled) subsequence, $\tilde{\varphi}_{n}$ converges weakly in $W^{1, q_{\Phi}}\left(\Omega ; \mathbb{R}^{d}\right)$ to some $\tilde{\varphi} \in \mathcal{M}(t, P)$ and, in addition, $B\left(\nabla \tilde{\varphi}_{n}, P_{n}\right) \rightarrow B(\nabla \tilde{\varphi}, P)$ in $L^{1}\left(\Omega ; \mathbb{R}^{d \times d}\right)$. Hence $\Xi=\mathrm{D} K(P)-\Delta_{q_{\mathrm{G}}} P+B(\nabla \tilde{\varphi}, P)$, i.e. $\tilde{\varphi} \in \mathfrak{R}(t, P, \Xi)$. Since $\left.\ell \in \mathrm{C}^{1}([0, T]) ; W^{1, q_{\Phi}}\left(\Omega ; \mathbb{R}^{d}\right)^{*}\right)$, we have

$$
\lim _{n \rightarrow \infty}-\left\langle\dot{\ell}\left(t_{n}\right), \tilde{\varphi}_{n}\right\rangle_{W^{1, q_{\Phi}}}=-\langle\dot{\ell}(t), \tilde{\varphi}\rangle_{W^{1, q_{\Phi}}} \leq \mathfrak{P}(t, P, \Xi)
$$

where the last inequality is due to the definition of $\mathfrak{P}(t, P, \Xi)$ in (4.3). From (4.58) we conclude the third relation in (4.44) and Lemma 4.9 is proved. 
Finally, it remains to verify condition 2 2. $\mathrm{E}_{2}$, i.e. the variational sum rule. For this, we apply Proposition 4.2 from [MRS13, which indeed holds for a general subdifferential $\mathfrak{F}$ and for a family $\left(\Psi_{P}\right)_{P \in \mathrm{D}}$ of dissipation potentials complying with $2 . \Psi_{1}$ and $\left(2 . \Psi_{2}\right)$. It states that, if for every $(t, P) \in[0, T] \times \mathrm{D}$ the set $\mathfrak{F}(t, P)$ contains the Fréchet subdifferential

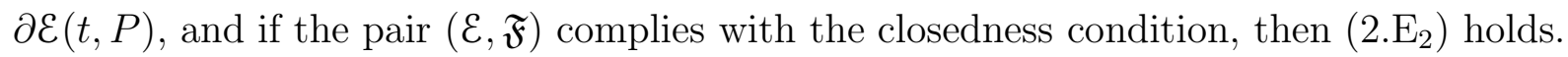
Hence, the following result is a consequence of Lemmas 4.4, 4.6 (guaranteeing the crucial subdifferential inclusion (4.36) ), and of Lemma 4.9.

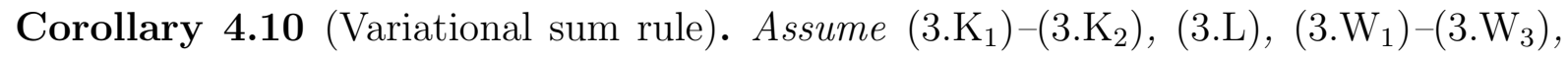
and (3.21) -(3.22). Then, the dissipation functionals $\left(\Psi_{z}\right)_{z \in \mathrm{D}}$ and the reduced energy functional $\mathcal{E}$ comply with the variational sum rule $\left(2 . \mathrm{E}_{2}\right)$.

\subsection{Conclusion of the proof of Theorem 3.3}

In view of Lemmas 4.24.9 and Corollary 4.10, our Theorem 2.7 applies to the generalized gradient system $(X, \mathcal{E}, \Psi, \mathfrak{F}, \mathfrak{P})$, yielding the existence of EDI solutions to the viscoplastic Cauchy problem in the form of the abstract doubly nonlinear equation (2.1). This finishes the proof of Theorem 3.3 .

Remark 4.11 (Missing chain rule). It remains an open problem to prove or disprove that the functional $\mathcal{E}$ with differentials $(\mathfrak{F}, \mathfrak{P})$ fulfills the CRI (2.15). Indeed, let a curve $P \in \mathrm{AC}\left([0, T] ; L^{p}\left(\Omega ; \mathbb{R}^{d \times d}\right)\right)$ and $\Xi \in L^{1}\left(0, T ; L^{p^{\prime}}\left(\Omega ; \mathbb{R}^{d \times d}\right)\right)$ fulfill (2.14). Then, $\dot{P} \in L^{p}\left(0, T ; L^{p}\left(\Omega ; \mathbb{R}^{d \times d}\right)\right)$ and $\Xi \in L^{p^{\prime}}\left(0, T ; ; L^{p^{\prime}}\left(\Omega ; \mathbb{R}^{d \times d}\right)\right)$, hence their duality pairing is well defined for almost all $t \in(0, T)$. But, in order to estimate it as required in (2.15) , one would have to use the full information provided by the structure of the marginal subdifferential, i.e. by the formula $\Xi=-\Delta_{q_{\mathrm{G}}} P+\mathrm{D} K(P)+B(\nabla \varphi, P)$ with $\varphi \in \mathcal{M}(t, P)$. Since there are no compensations between the terms contributing to $\Xi$, this would ultimately boil down to estimating individually the duality pairings $\left\langle-\Delta_{q_{\mathrm{G}}} P, \dot{P}\right\rangle,\langle\mathrm{D} K(P), \dot{P}\rangle$, and $\langle B(\nabla \varphi, P), \dot{P}\rangle$, which seems to be out of reach. In fact, the stress control condition (3.W 3 (ii) only ensures that $B(\nabla \varphi, P) \in L^{\infty}\left(0, T ; L^{1}\left(\Omega ; \mathbb{R}^{d \times d}\right)\right)$, and accordingly by a comparison argument we may only conclude that $-\Delta_{q_{\mathrm{G}}} P \in L^{\infty}\left(0, T ; L^{1}\left(\Omega ; \mathbb{R}^{d \times d}\right)\right)$. Hence, neither the first, nor the third duality pairings are well defined.

As we will see in Section 5, this problem can be circumvented if we add to the stored energy $\mathcal{J}$ a term which improves the spatial estimates for the Mandel stress tensor.

\section{Existence of energy solutions for a regularized sys- tem}

We now investigate an alternative model for finite-strain viscoplasticity, where the stored energy $\mathcal{J}$ from (3.9) is augmented by a regularizing contribution $\mathcal{J}_{3}$, multiplied by a (small, but positive) parameter $\eta>0$. We will show that the reduced energy $\varepsilon_{\eta}$ accordingly obtained by minimizing out the deformations complies with the CRI (2.15), in addition

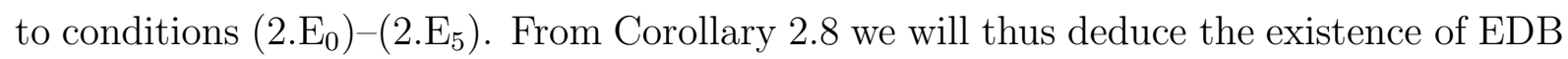
solutions to the generalized gradient system (2.1) driven by the regularized energy $\varepsilon_{\eta}$. 
This will yield solutions to a version of the PDE system (1.8) with a different minimum problem for $\varphi$, but the same flow rule for $P$. For this, it will be crucial that $\mathcal{J}_{3}$ in (5.3) below does not depend on the plastic variable $P$.

\subsection{The regularized model}

In the next lines, we specify the form of the regularizing contribution to the stored energy and accordingly introduce the energy functional driving the regularized gradient system.

\section{The regularized stored energy}

For $\eta>0$ fixed we define the functional $\mathcal{J}_{\eta}:[0, T] \times W^{1, q_{\Phi}}\left(\Omega ; \mathbb{R}^{d}\right) \times L^{p}\left(\Omega ; \mathbb{R}^{d \times d}\right) \rightarrow(-\infty, \infty]$ by

$$
\mathcal{J}_{\eta}(t, \varphi, P):=\mathcal{E}_{1}(P)+\mathcal{J}_{2}(t, \varphi, P)+\eta \mathcal{J}_{3}(\varphi),
$$

with $\mathcal{E}_{1}$ and $\mathcal{J}_{2}$ from (3.10) and (3.12) , respectively, and $\mathcal{J}_{3}: W^{1, q_{\Phi}}\left(\Omega ; \mathbb{R}^{d}\right) \rightarrow[0, \infty]$ defined by

$$
\mathcal{J}_{3}(\varphi):= \begin{cases}\int_{\Omega} \widetilde{W}(x, \nabla \varphi(x)) \mathrm{d} x & \text { if } \widetilde{W}(\cdot, \nabla \varphi) \in L^{1}(\Omega) \\ \infty & \text { otherwise. }\end{cases}
$$

Accordingly, the set of admissible deformations is now

$$
\widetilde{\mathcal{F}}:=\mathcal{F} \cap\left\{\varphi \in W^{1, q_{\Phi}}\left(\Omega ; \mathbb{R}^{d}\right): \widetilde{W}(\cdot, \nabla \varphi) \in L^{1}(\Omega)\right\} .
$$

We impose that the elastic energy $\widetilde{W}$ in $(\underline{5.2})$ is bounded from below, that

$$
\text { for all } x \in \Omega \text { the functional } \widetilde{W}(x, \cdot): \mathbb{R}^{d \times d} \rightarrow[0, \infty] \text { is polyconvex, }
$$

and that $\widetilde{W}$ controls $W$ in the following sense

$$
\begin{gathered}
\forall S>0 \exists C_{1}^{S}>0 \forall x \in \Omega \forall F, P \in \mathbb{R}^{d \times d} \text { with }|P|+\left|P^{-1}\right| \leq S: \\
\left|W\left(x, F P^{-1}\right)\right|^{p^{\prime}} \leq C_{1}^{S}(\widetilde{W}(x, F)+1) .
\end{gathered}
$$

Recall that $p^{\prime}=\frac{p}{p-1}$ is the conjugate exponent to $p$ : in fact, $5 . \widetilde{\mathrm{W}}_{2}$ ) is tuned to the coercivity/growth properties of the dissipation metric $R$.

Example 5.1. Condition $\left.5 . \widetilde{\mathrm{W}}_{2}\right)$ is satisfied if, for example, in addition to $3 . \mathrm{W}_{1}-3 . \mathrm{W}_{3}$ the functional $W$ fulfills for some $q_{W}>1$ the upper estimate

$$
\exists C_{6}>0 \forall x \in \Omega \forall F \in \mathrm{GL}^{+}(d): \quad W(x, F) \leq C_{6}\left(|F|^{q_{W}}+\left|F^{-1}\right|^{q_{W}}+1\right),
$$

whereas $\widetilde{W}$ is $\infty$ on $\mathbb{R}^{d \times d} \backslash \mathrm{GL}^{+}(d)$ and satisfies the coercivity estimate

$$
\exists C_{7}, C_{8}>0 \forall F \in \mathrm{GL}^{+}(d): \widetilde{W}(x, F) \geq C_{7}\left(|F|^{p^{\prime} q_{W}}+\left|F^{-1}\right|^{p^{\prime} q_{W}}\right)-C_{8} .
$$




\section{The regularized reduced energy}

We now introduce the energy functional obtained by minimizing out the deformations from $\mathcal{J}_{\eta}$, viz.

$$
\mathcal{E}_{\eta}(t, P):=\inf \left\{\mathcal{J}_{\eta}(t, \varphi, P): \varphi \in \widetilde{\mathcal{F}}\right\} \quad \text { for }(t, P) \in[0, T] \times X,
$$

with $X=L^{p}\left(\Omega ; \mathbb{R}^{d \times d}\right)$. Observe that $\operatorname{dom}\left(\mathcal{E}_{\eta}\right)=\operatorname{dom}(\mathcal{E})=[0, T] \times \mathrm{D}$, with $\mathrm{D}$ fulfilling (3.18). We also consider the reduced energy

$$
\mathcal{E}_{2, \eta}(t, P):=\inf \left\{\mathcal{J}_{2}(t, \varphi, P)+\eta \mathcal{J}_{3}(\varphi): \varphi \in \widetilde{\mathcal{F}}\right\}
$$

so that $\mathcal{E}_{\eta}(t, P)=\mathcal{E}_{1}(P)+\mathcal{E}_{2, \eta}(t, P)$.

\section{The marginal subdifferential}

Let $\mathfrak{F}_{\eta}:[0, T] \times X \rightrightarrows X^{*}$ be given by

$$
\mathfrak{F}_{\eta}(t, P)=\left\{\mathrm{D}_{P} \mathcal{J}_{\eta}(t, \varphi, P): \varphi \in \mathcal{M}_{\eta}(t, P)\right\},
$$

where $\mathcal{M}_{\eta}(t, P)$ is the set of minimizers for $\mathcal{J}_{\eta}(t, \cdot, P)$ over $\widetilde{\mathcal{F}}$. Since the functional $\mathcal{J}_{3}$ does not depend on $P$, we have

$$
\mathfrak{F}_{\eta}(t, P)=\left\{\mathrm{D}_{P} \mathcal{J}(t, \varphi, P): \varphi \in \mathcal{M}_{\eta}(t, P)\right\}=\mathrm{D}_{1}(P)+\left\{B(\cdot, \nabla \varphi, P): \varphi \in \mathcal{M}_{\eta}(t, P)\right\}
$$

Hence, the doubly nonlinear evolution equation

$$
\partial \Psi_{P}(\dot{P}(t))+\mathfrak{F}_{\eta}(t, P(t)) \ni 0 \quad \text { a.e. in }(0, T)
$$

associated with the generalized gradient system $\left(X, \mathcal{E}_{\eta}, \Psi, \mathfrak{F}_{\eta}, \mathfrak{P}_{\eta}\right)$ (with the dissipation potentials $\left(\Psi_{P}\right)_{P \in \mathrm{D}}$ from (3.20) and $\mathfrak{P}_{\eta}: \operatorname{graph}\left(\mathfrak{F}_{\eta}\right) \rightarrow \mathbb{R}$ defined by analogy with (4.3) $)$, yields a solution to the regularized PDE system

$$
\begin{aligned}
& \varphi(t) \in \operatorname{Argmin}\left\{\int_{\Omega} W\left(x, \nabla \widetilde{\varphi}(x) P^{-1}(t, x)\right)+\eta \widetilde{W}(x, \nabla \widetilde{\varphi}(x)) \mathrm{d} x: \widetilde{\varphi} \in \widetilde{\mathcal{F}}\right\}, \\
& 0 \in \partial R\left(x, \dot{P} P^{-1}\right) P^{-\mathrm{T}}-\Delta_{q_{\mathrm{G}}} P+\mathrm{D} K(P)+B(x, \nabla \varphi, P) \text { in } \Omega,
\end{aligned}
$$

where (5.9b) is supplemented with homogeneous Neumann boundary conditions for $P$ on $\partial \Omega$.

\section{Existence for the regularized system}

Our next result states the existence of EDB solutions for the Cauchy problem associated with system (5.9).

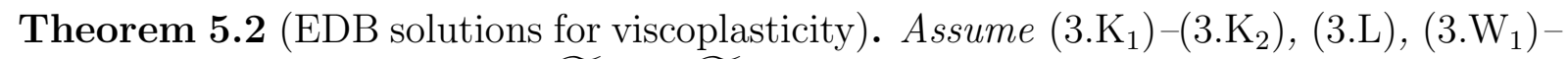
$\left(3 . \mathrm{W}_{3}\right)$, 3.R $\left.-3 . \mathrm{R}_{3}\right)$, and $\left.5 . \widetilde{\mathrm{W}}_{1}-3 . \widetilde{\mathrm{W}}_{2}\right)$. Suppose that $\eta>0$ and that the exponents

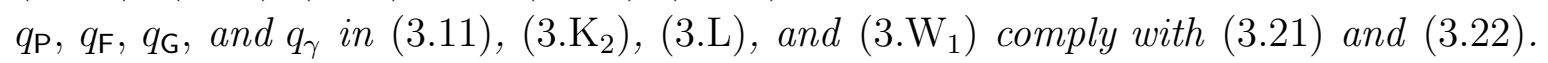

Then, for every initial datum $P_{0}$ as in (3.23) there exist $P \in L^{\infty}\left(0, T ; W^{1, q_{\mathrm{G}}}\left(\Omega ; \mathbb{R}^{d \times d}\right)\right) \cap$ $W^{1, p}\left(0, T ; L^{p}\left(\Omega ; \mathbb{R}^{d \times d}\right)\right)$ and $\varphi \in L^{\infty}\left(0, T ; W^{1, q_{\Phi}}\left(\Omega ; \mathbb{R}^{d}\right)\right)$ solving the Neumann boundary 
value problem for system (5.9) and complying with the initial condition $P(0, \cdot)=P_{0}(\cdot)$ a.e. in $\Omega$.

Moreover, the function $t \mapsto \mathcal{J}_{\eta}(t, P(t))$ is absolutely continuous on $[0, T]$ and, setting

$$
\Xi(t, x)=-\Delta_{q_{\mathrm{G}}} P(t, x)+\mathrm{D} K(P(t, x))+B(x, \nabla \varphi(t, x), P(t, x)),
$$

we have $\Xi \in L^{p^{\prime}}\left(0, T ; L^{p^{\prime}}\left(\Omega ; \mathbb{R}^{d \times d}\right)\right)$, and the pair $(P, \Xi)$ satisfies for all $0 \leq s \leq t \leq T$ the energy-dissipation balance

$$
\begin{aligned}
\mathcal{J}_{\eta}(t, \varphi(t), P(t))+\int_{s}^{t} \int_{\Omega} & \left(R\left(x, \dot{P}(r, x) P(r, x)^{-1}\right)+R^{*}\left(x,-\Xi(r, x) P(r, x)^{\top}\right)\right) \mathrm{d} x \mathrm{~d} r \\
= & \mathcal{J}_{\eta}(s, \varphi(s), P(s))-\int_{s}^{t}\langle\dot{\ell}(r), \varphi(r)\rangle_{W^{1, q_{\Phi}}} \mathrm{d} r .
\end{aligned}
$$

\subsection{Proof of Theorem 5.2}

In what follows, we will verify that the regularized energy $\varepsilon_{\eta}$ complies with the conditions of Corollary 2.8, again omitting to denote the $x$-dependence of $W$ and $R$, and the related quantities, in the proofs of the various lemmas ahead.

The analogs of Lemmas 4.1, 4.2, and 4.3 hold for the regularized energy $\mathcal{E}_{\eta}$, which

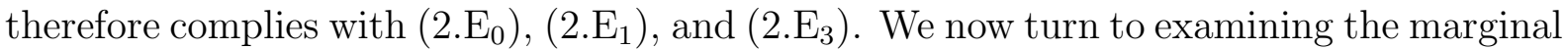
subdifferential $\mathfrak{F}_{\eta}$. With the following result we prove that $\mathfrak{F}_{\eta}(t, P)$ contains the Fréchet subdifferential $\partial \mathcal{E}_{\eta}(t, P)$ at every $(t, P) \in[0, T] \times$ D. Lemma 5.3 below also features an $L^{p^{\prime}}\left(\Omega ; \mathbb{R}^{d \times d}\right)$-estimate of the backstress contribution $B$ to $\mathfrak{F}_{\eta}(t, P)$ (cf. (5.11) ahead, to be compared with estimate (4.37) in Lemma 4.6), and a sort of uniform superdifferentiability property for the regularized energy $\mathcal{E}_{\eta}$, cf. (5.12) ahead. Once again, for its proof we resort to Lemma 4.5. Estimate (5.12) will play a crucial role in the proof of the CRI (2.15), cf. Lemma 5.4 ahead.

Lemma 5.3 (Properties of the marginal subdifferential $\mathfrak{F}_{\eta}$ for $\left.\mathcal{E}_{\eta}\right)$. Assume $3 . \mathrm{K}_{1}$ - (3.K.

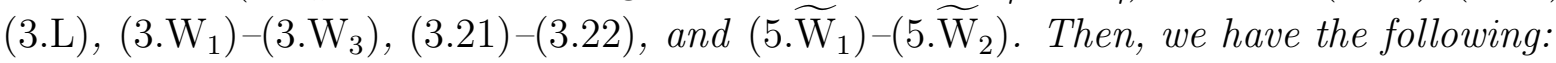

1. For every $(t, P) \in[0, T] \times \mathrm{D}$ and $\varphi \in \mathcal{M}_{\eta}(t, P)$ we have $B(\nabla \varphi, P) \in L^{p^{\prime}}\left(\Omega ; \mathbb{R}^{d \times d}\right)$, and

$$
\begin{aligned}
\exists \tilde{c}_{7}>0 & \forall(t, P) \in[0, T] \times \mathrm{D} \forall \varphi \in \mathcal{M}_{\eta}(t, P): \\
& \|B(\nabla \varphi, P)\|_{L^{p^{\prime}}} \leq \tilde{c}_{7}\left\|P^{-1}\right\|_{L^{\infty}}\left(\mathcal{G}_{\eta}(P)+1\right) .
\end{aligned}
$$

2. For every $(t, P) \in[0, T] \times \mathrm{D}$ we have $\partial \mathcal{E}_{\eta}(t, P) \subset \mathfrak{F}_{\eta}(t, P)$.

3. There exists $\theta \in(0,1)$ and for all $S>0$ there exist constants $C_{3}^{S}>0$ and $r^{*}>0$ such that for every $P_{1}, P_{2} \in \mathrm{D}$ such that

$$
\max \left\{\mathcal{G}_{\eta}\left(P_{1}\right), \mathcal{G}_{\eta}\left(P_{2}\right)\right\} \leq S \text { and }\left\|P_{1}-P_{2}\right\|_{L^{p}} \leq r^{*}
$$

and for all $\varphi_{1} \in \mathcal{M}_{\eta}\left(t, P_{1}\right)$ there holds

$$
\mathcal{E}_{2, \eta}\left(t, P_{2}\right)-\mathcal{E}_{2, \eta}\left(t, P_{1}\right)-\int_{\Omega} B\left(\nabla \varphi_{1}, P_{1}\right):\left(P_{2}-P_{1}\right) \mathrm{d} x \leq C_{2}^{S}\left\|P_{2}-P_{1}\right\|_{L^{p}}^{2-\theta} .
$$


Proof. Ad (1): The proof follows using the argument developed for (4.36).

Ad (2): For the proof of (5.11), it is sufficient to combine $\left.3 . \mathrm{W}_{3}\right)$ (ii) and $\left(5 . \widetilde{\mathrm{W}}_{2}\right)$, viz.

$$
\begin{aligned}
& \int_{\Omega}|B(\nabla \varphi(x), P(x))|^{p^{\prime}} \mathrm{d} x \leq C \int_{\Omega}\left(W\left(\nabla \varphi(x) P(x)^{-1}\right)+1\right)^{p^{\prime}}\left|P(x)^{-\mathrm{T}}\right|^{p^{\prime}} \mathrm{d} x \\
& \leq C \int_{\Omega}(\widetilde{W}(\nabla \varphi(x))+1)\left|P(x)^{-\mathrm{\top}}\right|^{p^{\prime}} \mathrm{d} x \leq C\left\|P^{-1}\right\|_{L^{\infty}\left(\Omega ; \mathbb{R}^{\prime} \times d\right)}^{p^{\prime}}\left(\mathcal{E}_{2, \eta}(t, P)+1\right) .
\end{aligned}
$$

Ad (3): Let $P_{1}, P_{2} \in \mathrm{D}$ comply with (15.12a) and let $\varphi_{1} \in M_{\eta}\left(t, P_{1}\right)$. We have

$$
\begin{aligned}
& \mathcal{E}_{2, \eta}\left(t, P_{2}\right)-\mathcal{E}_{2, \eta}\left(t, P_{1}\right) \\
& \quad \leq \int_{\Omega}\left(W\left(\nabla \varphi P_{2}^{-1}\right)-W\left(\nabla \varphi P_{1}^{-1}\right)-B\left(\nabla \varphi, P_{1}\right)\left(P_{2}-P_{1}\right)\right) \mathrm{d} x \\
& \quad \stackrel{(1)}{\leq} C_{W} \int_{\Omega}\left(W\left(\nabla \varphi P_{1}^{-1}\right)+1\right)\left|P_{1}-P_{2}\right|^{2} \mathrm{~d} x \\
& \quad \stackrel{(2)}{\leq} C_{W}\left\|W\left(\nabla \varphi P_{1}^{-1}\right)+1\right\|_{L^{p^{\prime}}}\left\|P_{1}-P_{2}\right\|_{L^{p}}\left\|P_{1}-P_{2}\right\|_{L^{\infty}} \\
& \quad \stackrel{(3)}{\leq} C\left\|\widetilde{W}\left(\nabla \varphi_{1}\right)+1\right\|_{L^{1}}^{1 / p^{\prime}}\left\|P_{1}-P_{2}\right\|_{L^{p}}^{2-\theta}\left\|P_{1}-P_{2}\right\|_{W^{1, q_{G}}}^{\theta} \stackrel{(4)}{\leq} C\left\|P_{1}-P_{2}\right\|_{L^{p}}^{2-\theta} .
\end{aligned}
$$

Here, (1) is derived via estimate (4.30) from Lemma 4.5 as follows. First we use (5.12a) giving the upper bounds

$$
\left\|P_{1}\right\|_{W^{1, q_{\mathrm{G}}}}+\left\|P_{2}\right\|_{W^{1, q_{\mathrm{G}}}} \leq C_{3}^{S}
$$

by the analog of (4.16). Using $q_{\mathrm{G}}>d$ the Gagliardo-Nirenberg inequality

$$
\left\|P_{1}-P_{2}\right\|_{L^{\infty}} \leq C_{\mathrm{GN}}\left\|P_{1}-P_{2}\right\|_{W^{1, q_{\mathrm{G}}}}^{\theta}\left\|P_{1}-P_{2}\right\|_{L^{p}}^{1-\theta} \quad \text { with } \theta=\frac{d q_{\mathrm{G}}}{q_{\mathrm{G}} p-d p+d q_{\mathrm{G}}} \in(0,1)
$$

provides smallness of $\left\|P_{1}-P_{2}\right\|_{L^{\infty}}$. Indeed, choosing $r^{*}=\frac{1}{2}\left(\tilde{r} /\left(2 C_{3}^{S}\right)^{\theta}\right)^{1 /(1-\theta)}$ with $\tilde{r}$ from (4.30), we find $\left\|P_{1}-P_{2}\right\|_{L^{\infty}} \leq\left(2 C_{3}^{S}\right)^{\theta}\left(r^{*}\right)^{1-\theta}<\tilde{r}$, such that (4.30) is applicable.

Estimate (2) is Hölder's inequality, while to obtain (3) we use estimate $5 . \widetilde{\mathrm{W}}_{2}$, observing that $\left\|P_{1}\right\|_{L^{\infty}}+\left\|P_{1}^{-1}\right\|_{L^{\infty}} \leq C$ due to (5.15) and the continuous embeddings $W^{1, q_{\mathrm{G}}}\left(\Omega ; \mathbb{R}^{d \times d}\right) \subset L^{\infty}\left(\Omega ; \mathbb{R}^{d \times d}\right)$ and $W^{1, \tilde{q}}\left(\Omega ; \mathbb{R}^{d \times d}\right) \subset L^{\infty}\left(\Omega ; \mathbb{R}^{d \times d}\right)$. We also again resort the Gagliardo-Nirenberg inequality (5.16). Finally, (4) ensues from the energy bound $S \geq \mathcal{G}_{\eta}\left(P_{1}\right) \geq c\left\|\widetilde{W}\left(\nabla \varphi_{1}\right)+1\right\|_{L^{1}}-C$ by the analog of (4.15) , and again from the bound (5.15). We have thus established (5.12b).

With the very same argument as in the proofs of Lemmas 4.8 and 4.9 it is possible to check conditions $\left(2 . \mathrm{E}_{4}\right)$ and $\left(2 . \mathrm{E}_{5}\right)$, which implies the variational sum rule $\left(2 . \mathrm{E}_{2}\right)$. We now check the CRI (2.15).

Lemma 5.4 (Chain-rule inequality for the regularized energy $\mathcal{E}_{\eta}>0$ ). Assume that $\left(3 . \mathrm{K}_{1}\right)-\left(3 . \mathrm{K}_{2}\right),(3 . \mathrm{L}),\left(3 . \mathrm{W}_{1}\right)-\left(3 . \mathrm{W}_{3}\right),(3.21)-(3.22)$, and $5 . \widetilde{\mathrm{W}}_{1}-\left(5 . \widetilde{\mathrm{W}}_{2}\right)$ hold. Then, the triple $\left(\mathcal{E}_{\eta}, \mathfrak{F}_{\eta}, \mathfrak{P}_{\eta}\right)$ fulfills the CRI (2.15). 
Proof. Let us fix a curve $P \in \mathrm{AC}\left([0, T] ; L^{p}\left(\Omega ; \mathbb{R}^{d \times d}\right)\right)$ and a function $\Xi \in L^{1}\left(0, T ; L^{p^{\prime}}\left(\Omega ; \mathbb{R}^{d \times d}\right)\right)$ fulfilling (2.14). Taking into account (4.15), (4.16), and (4.23), we have a fortiori that

$$
\begin{aligned}
& P \in L^{\infty}\left(0, T ; W^{1, q_{\mathrm{G}}}\left(\Omega ; \mathbb{R}^{d \times d}\right)\right) \cap W^{1, p}\left(0, T ; L^{p}\left(\Omega ; \mathbb{R}^{d \times d}\right)\right), \\
& P^{-1} \in L^{\infty}\left(0, T ; W^{1, \tilde{q}}\left(\Omega ; \mathbb{R}^{d \times d}\right)\right), \\
& \Xi \in L^{p^{\prime}}\left(0, T ; L^{p^{\prime}}\left(\Omega ; \mathbb{R}^{d \times d}\right)\right)
\end{aligned}
$$

with

$$
\Xi(t)=-\Delta_{q_{\mathrm{G}}} P(t)+\mathrm{D} K(P(t))+B(\nabla \varphi(t), P(t)) \quad \text { for a.a. } t \in(0, T) .
$$

Here, $t \mapsto \varphi(t) \in \mathcal{M}_{\eta}(t, P(t))$ is a measurable selection. Each of the three terms on the right-hand side of $(\underline{5.18})$ is in $L^{p^{\prime}}\left(0, T ; L^{p^{\prime}}\left(\Omega ; \mathbb{R}^{d \times d}\right)\right)$ and in fact we have

$$
\begin{aligned}
& \left\|-\Delta_{q_{\mathrm{G}}} P\right\|_{L^{p^{\prime}\left(0, T ; L^{p^{\prime}}\left(\Omega ; \mathbb{R}^{d \times d}\right)\right)}}+\|\mathrm{D} K(P)\|_{L^{\infty}\left(0, T ; L^{\infty}\left(\Omega ; \mathbb{R}^{d \times d}\right)\right)} \\
& +\|B(\nabla \varphi, P)\|_{L^{\infty}\left(0, T ; L^{p^{\prime}}\left(\Omega ; \mathbb{R}^{d \times d}\right)\right)} \leq C .
\end{aligned}
$$

The estimate for $B(\nabla \varphi, P)$ follows from (5.11) using $\sup _{t \in[0, T]} \varepsilon_{\eta}(t, P(t))<\infty$. The bound for $\mathrm{D} K(P)$ is due to the fact that

$$
P \in \mathrm{C}^{0}\left([0, T] ; \mathrm{C}^{0}\left(\bar{\Omega} ; \mathbb{R}^{d \times d}\right)\right) \text {, which in particular implies } \operatorname{det}(P(t, x)) \geq \pi>0,
$$

(where we have used the compact embedding $W^{1, q_{\mathrm{G}}}\left(\Omega ; \mathbb{R}^{d \times d}\right) \Subset \mathrm{C}^{0}\left(\bar{\Omega} ; \mathbb{R}^{d \times d}\right)$ ), combined with the fact that $\mathrm{D} K$ is continuous on $\mathrm{GL}^{+}(d)$. Then, the estimate for $-\Delta_{q_{\mathrm{G}}} P$ ensues from a comparison argument, using $\Xi \in L^{p^{\prime}}\left(0, T ; L^{p^{\prime}}\left(\Omega ; \mathbb{R}^{d \times d}\right)\right)$.

Now, the chain rule for $\mathcal{E}^{1}$, given by the sum of a convex and of a Fréchet differentiable functional, yields that

$$
\begin{aligned}
& \text { the map } t \mapsto \mathcal{E}^{1}(t, P(t)) \text { is absolutely continuous and } \\
& \frac{\mathrm{d}}{\mathrm{d} t} \mathcal{E}^{1}(t, P(t))=\int_{\Omega}\left(-\Delta_{q_{\mathrm{G}}} P(t)+\mathrm{D} K(P(t))\right): \dot{P}(t) \mathrm{d} x \quad \text { for a.a. } t \in(0, T) .
\end{aligned}
$$

Let us now prove that the map $t \mapsto \mathcal{E}_{2, \eta}(t, P(t))$ is absolutely continuous, by estimating the difference $\left|\mathcal{E}_{2, \eta}(t, P(t))-\mathcal{E}_{2, \eta}(s, P(s))\right|$. We will use that $\sup _{t \in[0, T]} \mathcal{G}_{\eta}(P(t)) \leq S$. Without loss of generality, we may also suppose that $\|P(t)-P(s)\|_{L^{p}} \leq r^{*}$ with $r^{*}>0$ from (5.12a). We have

$$
\begin{aligned}
& \mathcal{E}_{2, \eta}(t, P(t))-\mathcal{E}_{2, \eta}(s, P(s)) \\
& =\mathcal{E}_{2, \eta}(t, P(t))-\mathcal{E}_{2, \eta}(t, P(s))+\mathcal{E}_{2, \eta}(t, P(s))-\mathcal{E}_{2, \eta}(s, P(s)) \\
& \leq \mathcal{E}_{2, \eta}(t, P(t))-\mathcal{E}_{2, \eta}(t, P(s))+\mathcal{J}_{2}(t, \varphi(s), P(s))-\mathcal{J}_{2}(s, \varphi(s), P(s)) \\
& \stackrel{(1)}{\leq} \int_{\Omega} B(\nabla \varphi(s), P(s)):(P(t)-P(s)) \mathrm{d} x \\
& \quad \quad+C_{2}^{S}\|P(t)-P(s)\|_{L^{p}}^{2-\theta}-\langle\ell(t)-\ell(s), \varphi(s)\rangle_{W^{1, q_{\Phi}}},
\end{aligned}
$$

where $\varphi(s)$ is a selection in $\mathcal{M}_{\eta}(s, P(s))$, and for (1) we have used estimate (5.12b). Exchanging the role of $s$ and $t$, we thus conclude, for every $0 \leq s \leq t \leq T$, the estimate 


$$
\begin{aligned}
& \left|\mathcal{E}_{2, \eta}(t, P(t))-\mathcal{E}_{2, \eta}(s, P(s))\right| \\
& \leq\|P(t)-P(s)\|_{L^{p}}\left(C_{2}^{S}\|P(t)-P(s)\|_{L^{p}}^{1-\theta}+\sup _{t \in[0, T]}\|B(\nabla \varphi(t), P(t))\|_{L^{p^{p}}}\right) \\
& +\|\ell(t)-\ell(s)\|_{W^{1, q_{\Phi}\left(\Omega ; \mathbb{R}^{d}\right)^{*}}}\left(\|\varphi(t)\|_{W^{1, q_{\Phi}}}+\|\varphi(s)\|_{W^{1, q_{\Phi}}}\right) \\
& \stackrel{(2)}{\leq} C\left(\|P(t)-P(s)\|_{L^{p}}+|t-s|\right) .
\end{aligned}
$$

Indeed, for (2) we have used condition (3.L) on $\ell$, the coercivity property (4.8) for $\mathcal{J}_{2}$, and combined (5.11) with estimate (4.16), so that

$$
\sup _{t \in[0, T]}\|B(\nabla \varphi(t), P(t))\|_{L^{p^{\prime}}} \leq \tilde{c}_{7} \sup _{t \in[0, T]}\left\|P(t)^{-1}\right\|_{L^{\infty}}\left(\mathcal{G}_{\eta}(P(t))+1\right) \leq C .
$$

Thus, from (5.23) and the fact that $P \in \mathrm{AC}\left([0, T] ; L^{p}\left(\Omega ; \mathbb{R}^{d \times d}\right)\right)$ we infer that $t \mapsto$ $\mathcal{E}_{2, \eta}(t, P(t))$ is absolutely continuous on $[0, T]$.

In order to prove the CRI (2.15), let us fix $t \in(0, T)$ outside a negligible set such that formula (5.21) holds and that for $h \downarrow 0$ we have

$$
\left\{\begin{array}{l}
\exists \frac{\mathrm{d}}{\mathrm{d} t} \mathcal{E}_{2, \eta}(t, P(t)), \\
\exists \dot{P}(t) \text { and } \frac{1}{h}(P(t+h)-P(t)) \rightarrow \dot{P}(t) \text { in } L^{p}\left(\Omega ; \mathbb{R}^{d \times d}\right), \\
\exists \dot{\ell}(t) \text { and } \frac{1}{h}(\ell(t+h)-\ell(t)) \rightarrow \dot{\ell}(t) \text { in } W^{1, q_{\Phi}}\left(\Omega ; \mathbb{R}^{d}\right)^{*} .
\end{array}\right.
$$

We now use estimate (5.22) for $\tilde{\varphi} \in \mathfrak{R}_{\eta}(t, P(t), \Xi(t))$, divide by $h<0$, and obtain

$$
\begin{aligned}
& \frac{1}{h}\left(\mathcal{E}_{2, \eta}(t+h, P(t+h))-\mathcal{E}_{2, \eta}(t, P(t))\right) \\
& \geq \frac{1}{h} \int_{\Omega} B(\nabla \tilde{\varphi}(t), P(t)):(P(t+h)-P(t)) \mathrm{d} x+C_{2}^{S} \frac{\|P(t+h)-P(t)\|_{L^{p}}^{2-\theta}}{h} \\
& \quad-\frac{1}{h}\langle\ell(t+h)-\ell(t), \tilde{\varphi}\rangle_{W^{1, q_{\Phi}}} .
\end{aligned}
$$

Taking the limit as $h \uparrow 0$ in the above inequality, we observe that the second term on the right-hand side converges to 0 by the second of (5.24) giving $\|P(t+h)-P(t)\| \leq 2\|h \dot{P}(t)\|$ for small $h$ and by $2-\theta>1$. Thus, for all $\tilde{\varphi} \in \mathfrak{R}_{\eta}(t, P(t), \Xi(t))$ we have

$$
\frac{\mathrm{d}}{\mathrm{d} t} \varepsilon_{2, \eta}(t, P(t)) \geq \int_{\Omega} B(\nabla \tilde{\varphi}, P(t)): \dot{P}(t) \mathrm{d} x-\langle\dot{\ell}(t), \tilde{\varphi}\rangle_{W^{1, q_{\Phi}}} .
$$

It follows from the definition of $\mathfrak{R}_{\eta}(t, P(t), \Xi(t))$ that

$$
B\left(\nabla \tilde{\varphi}_{1}, P(t)\right)=B\left(\nabla \tilde{\varphi}_{2}, P(t)\right) \quad \text { for all } \tilde{\varphi}_{1}, \tilde{\varphi}_{2} \in \mathfrak{R}_{\eta}(t, P(t), \Xi(t)) \text {. }
$$

Therefore, we have

$$
\frac{\mathrm{d}}{\mathrm{d} t} \mathcal{E}_{2, \eta}(t, P(t)) \geq \int_{\Omega} B(\nabla \varphi(t), P(t)): \dot{P}(t) \mathrm{d} x+\mathfrak{P}_{\eta}(t, P(t), \Xi(t)),
$$

for every selection $t \in(0, T) \mapsto \varphi(t) \in \mathfrak{R}_{\eta}(t, P(t), \Xi(t))$. Combining (5.21) and (5.25) yields the CRI (2.15). 
Remark 5.5. It is natural to wonder whether EDB solutions to the doubly nonlinear evolution equation (5.8) converge to a solution of the generalized gradient system driven by the original energy $\mathcal{E}$ from (3.16)), as the regularizing parameter $\eta$ vanishes. This is far from guaranteed. Indeed, mimicking the variational arguments that we have employed for passing to the time-continuous limit in the proof of Theorem 2.7, it should be possible to show that EDB solutions to (5.8) converge to a solution of the generalized gradient system driven by the energy functional $\widetilde{\mathcal{E}}(t, P):=\inf \left\{\mathcal{J}_{0}(t, \varphi, P): \varphi \in \widetilde{\mathcal{F}}\right\}$, with $\mathcal{J}_{0}(t, \cdot, P)$ the $\Gamma$-limit as $\eta \downarrow 0$ of $\mathcal{J}_{\eta}(t, \cdot, P)$ and $\widetilde{\mathcal{F}}$ the set of admissible deformations from (5.3). Note that $\widetilde{\mathcal{E}}$ need not coincide with $\mathcal{E}$, as there might be a Lavrentiev phenomenon.

\section{Extensions}

In this section we briefly discuss directions in which our analysis could be extended.

First, as in [MaM09] we might easily couple the evolution of the plastic variable $P$ with the (rate-dependent) evolution of some other hardening variable $p \in \mathbb{R}^{m}$, with $m \geq 1$.

Second, as outlined in MaM09, it would be possible to encompass in our model also a global version of the non-self-interpenetration condition, as proposed in Cia82. For this, it would be sufficient to replace the space of admissible deformations $\mathcal{F}$ by

$$
\begin{gathered}
\mathcal{F}_{\text {nsi }}=\left\{\varphi \in W^{1, q_{\Phi}}\left(\Omega ; \mathbb{R}^{d}\right): \varphi=\varphi_{\text {Dir }} \text { on } \Gamma_{\text {Dir }}, \operatorname{det} \nabla \varphi \geq 0 \text { a.e. in } \Omega,\right. \\
\left.\int_{\Omega} \operatorname{det}(\nabla \varphi) \mathrm{d} x \leq \operatorname{vol}(y(\Omega))\right\} .
\end{gathered}
$$

Since $\mathcal{F}_{\text {nsi }}$ is weakly closed in $W^{1, q_{\Phi}}\left(\Omega ; \mathbb{R}^{d}\right)$ if $q_{\Phi}>d$, it is possible to define our reduced energy by minimizing out from the stored energy the deformations in $\mathcal{F}_{\text {nsi }}$. Then, all our results carry over to this case.

In what follows, we will focus more specifically on the extension to time-dependent Dirichlet loadings. To replace the time-independent Dirichlet condition $\varphi(t, x)=\varphi_{\text {Dir }}(x)$ for $(t, x) \in[0, T] \times \Gamma_{\text {Dir }}$ by

$$
\varphi(t, x)=g_{\text {Dir }}(t, x) \quad(t, x) \in[0, T] \times \Gamma_{\text {Dir }},
$$

with $g_{\text {Dir }}:[0, T] \times \Gamma_{\text {Dir }} \rightarrow \mathbb{R}^{d}$ given, we follow the ideas from [FrM06, Sec. 5].

For this, we will suppose that $g_{\text {Dir }}$ can be extended to $[0, T] \times \mathbb{R}^{d}$ and search for the deformation $\varphi:[0, T] \times \Omega \rightarrow \mathbb{R}^{d}$ in the form of the composition

$$
\varphi(t, x)=g_{\text {Dir }}(t, y(t, x)) \quad \text { with } y(t, \cdot) \in y:=\left\{y \in W^{1, q_{\Phi}}\left(\Omega ; \mathbb{R}^{d}\right): y=\operatorname{Id} \text { on } \Gamma_{\text {Dir }}\right\} .
$$

In fact, underlying (6.3) is the implicit idea that $g_{\text {Dir }}(t, \cdot): \mathbb{R}^{d} \rightarrow \mathbb{R}^{d}$ is a diffeomorphism. Therefore, as in [FrM06, eqn. (5.7)] we require that $g_{\text {Dir }}$ satisfies

$$
\begin{aligned}
& g_{\text {Dir }} \in \mathrm{C}^{1}\left([0, T] \times \mathbb{R}^{d} ; \mathbb{R}^{d}\right), \quad \nabla g_{\text {Dir }} \in \mathrm{BC}^{1}\left([0, T] \times \mathbb{R}^{d} ; \mathbb{R}^{d \times d}\right), \\
& \exists C_{9}>0 \quad \forall(t, x) \in[0, T] \times \mathbb{R}^{d}: \quad\left|\nabla g_{\text {Dir }}(t, x)^{-1}\right| \leq C_{9},
\end{aligned}
$$

where BC stands for bounded continuous. 
We now rewrite the stored and the reduced energy functionals in terms of the variable $y$, taking into account that the composition (6.3) leads to the following multiplicative split for the deformation gradient

$$
\nabla \varphi(t, x)=\nabla g_{\text {Dir }}(t, y(t, x)) \nabla y(t, x) \quad \text { for every }(t, x) \in[0, T] \times \Omega .
$$

With a slight abuse of notation, we will continue to use the symbols $\mathcal{J}$ and $\mathcal{E}$ for

$$
\begin{aligned}
& \mathcal{J}(t, y, P):=\mathcal{E}_{1}(P)+\mathcal{J}_{2}(t, \varphi, P) \quad \text { with } \\
& \mathcal{J}_{2}(t, \varphi, P):=\left\{\begin{array}{cc}
\int_{\Omega} W\left(x, \nabla g_{\text {Dir }}(t, y) \nabla y P^{-1}\right) \mathrm{d} x & \text { if }(y, P) \in \mathcal{Y} \times \mathcal{P}, \\
\infty \quad\left\langle\ell(t), g_{\text {Dir }}(t, y)\right\rangle_{W^{1, q_{\Phi}}} & \text { otherwise, }
\end{array}\right. \\
& \mathcal{E}(t, P):=\inf \{\mathcal{J}(t, y, P): y \in y\}=\mathcal{E}_{1}(P)+\mathcal{E}_{2}(t, P) \\
& \quad \text { with } \mathcal{E}_{2}(t, P):=\inf \left\{\mathcal{J}_{2}(t, y, P): y \in y\right\} .
\end{aligned}
$$

Clearly, the marginal subdifferential of $\mathcal{E}$ is now given by

$$
\mathfrak{F}(t, P):=-\Delta_{q_{\mathrm{G}}} P+\mathrm{D} K(P)+\left\{B\left(\cdot, \nabla g_{\mathrm{Dir}}(t, y) \nabla y, P\right): y \in \mathcal{M}(t, P)\right\},
$$

where we still use the notation $\mathcal{M}(t, P)$ for $\operatorname{Argmin}_{y \in y} \mathcal{J}(t, y, P)$.

In what follows, we will discuss the conditions the gradient system $(X, \mathcal{E}, \Psi, \mathfrak{F}, \mathfrak{P})$, with $X=L^{p}\left(\Omega ; \mathbb{R}^{d \times d}\right)$, needed to obtain the abstract conditions $\left(2 . \mathrm{E}_{0}\right)-\left(2 . \mathrm{E}_{5}\right)$. The lower semicontinuity and coercivity $\left.2 . \mathrm{E}_{0}\right)$ and $\left(2 . \mathrm{E}_{1}\right)$ can be checked by suitably adapting the proofs of Lemmas 4.2 and 4.3, cf. also the arguments in the proofs of [FrM06, Lemma 5.5]. We now closely examine the time dependence of $\mathcal{E}$ and the properties of the power function $\mathfrak{P}$.

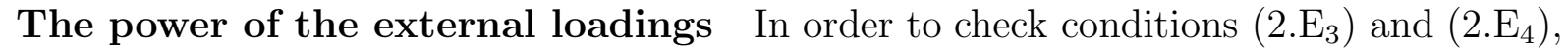
we will resort to the explicit calculation of the power $\partial_{t} \mathcal{J}(t, y, P)$ from [FrM06, Lemma 5.5]. Therein, it was proved that, in the case of zero volume and surface loadings (i.e. for $\ell=0)$

$$
\partial_{t} \mathcal{J}(t, y, P)=\partial_{t} \mathcal{J}_{2}(t, y, P)=\int_{\Omega} \mathbb{K}\left(x, \nabla g_{\text {Dir }}(t, y(x)) \nabla y(x) P(x)^{-1}\right): V(t, y(x)) \mathrm{d} x,
$$

where $S$ denotes the (multiplicative) Kirchhoff stress tensor

$$
\mathbb{K}(x, F):=\partial_{F} W(x, F) F^{\top}
$$

and we have used the short-hand notation $V(t, y) ;=\nabla \dot{g}_{\text {Dir }}(t, y)\left(\nabla g_{\text {Dir }}(t, y)\right)^{-1}$. Formula (6.9) was established under stress control conditions for $S$ of the same type as the ones in $3 . \mathrm{W}_{3}$, namely

$\exists \delta>0 \quad \exists \widetilde{C}_{4}, \widetilde{C}_{5}>0 \quad \forall(x, F) \in \operatorname{dom}(W) \forall N \in \mathcal{N}_{\delta}:$

(i) $W(x, \cdot): \mathrm{GL}^{+}(d) \rightarrow \mathbb{R}$ is differentiable,

(ii) $\left|\partial_{F} W(x, F) F^{\top}\right| \leq \widetilde{C}_{4}(W(x, F)+1)$,

(iii) $\left|\mathrm{D}_{F} W(x, F) F^{\top}-\mathrm{D}_{F} W(x, N F)(N F)^{\top}\right| \leq \widetilde{C}_{5}|N-\mathbf{1}|(W(x, F)+1)$.

Therefore, we have the following explicit formula and properties for $\partial_{t} \mathcal{J}$. 
Lemma 6.1. Assume (3.L), that $W$ is frame-indifferent and that it fulfills $3 . \mathrm{W}_{1}-3 . \mathrm{W}_{3}$

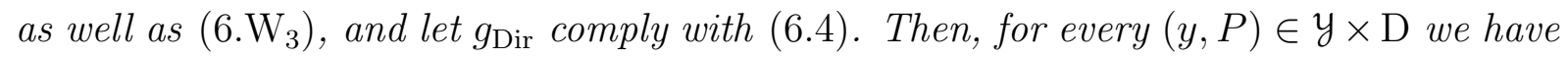

$$
\begin{aligned}
\partial_{t} \mathcal{J}(t, y, P)= & \int_{\Omega} \mathbb{K}\left(x, \nabla g_{\text {Dir }}(t, y(x)) \nabla y(x) P(x)^{-1}\right): V(t, y(x)) \mathrm{d} x \\
& -\left\langle\dot{\ell}(t), g_{\text {Dir }}(t, y)\right\rangle_{W^{1, q_{\Phi}}}-\left\langle\ell(t), \dot{g}_{\text {Dir }}(t, y)\right\rangle_{W^{1, q_{\Phi}}}
\end{aligned}
$$

and there exist $c_{9}, c_{10}>0$ and a modulus of continuity $\omega$ such that for every $s, t \in[0, T]$

$$
\begin{aligned}
& \left|\partial_{t} \mathcal{J}(t, y, P)\right| \leq c_{9}(\mathcal{J}(t, y, P)+1), \\
& \left|\partial_{t} \mathcal{J}(t, y, P)-\partial_{t} \mathcal{J}(s, y, P)\right| \leq \omega(|t-s|)\left(\mathcal{J}(t, y, P)+c_{10}\right) .
\end{aligned}
$$

Hence, for every $s, t \in[0, T]$ and every $(y, P) \in \boldsymbol{y} \times \mathrm{D}$ there holds

$$
\mathcal{J}(t, y, P)+1 \leq \exp \left(c_{9}|t-s|\right)(\mathcal{J}(s, y, P)+1)
$$

Proof. In the case $\ell=0$, i.e. when $\partial_{t} \mathrm{~J}$ is given by (6.9), formulae (6.12) and (6.13) were proved in [MaM09, Thm. 5.3]. In view of conditions (3.L), 6.W. , and (6.4), It is easy to check that they extend to the case when (6.11) holds. Hence, (6.14) follows via a simple Gronwall argument.

Then, the time-dependence estimate $\left.2 . \mathrm{E}_{3}\right)$ is an immediate consequence of (6.14), whereas $\left(2 . \mathrm{E}_{4}\right)$ can be checked straightforwardly, also resorting to $\left.6 . \mathrm{W}_{3}\right)$.

Corollary 6.2. Assume (3.L), that $W$ is frame-indifferent and fulfills $\left(3 . \mathrm{W}_{1}\right)-\left(3 . \mathrm{W}_{3}\right)$

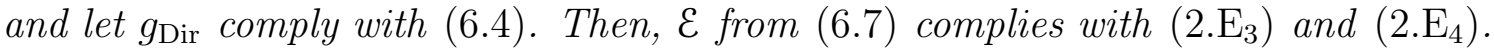

To establish the closedness condition (2.E5), we exploit (6.4) to check that for $\mathfrak{F}$ from (6.8) the analog of Lemma 4.6 holds. Furthermore, a combination of the arguments from the proof of Lemma 4.9 with the techniques from [MaM09, Prop. 5.1, Thm. 5.2] allows us to prove that $(\mathcal{E}, \mathfrak{F})$ comply with the closedness property in $2 . \mathrm{E}_{5}$ ) (whence the variational sum rule $\left(2 . \mathrm{E}_{2}\right)$ ).

Hence, it remains to check the upper semicontinuity of the functional $\mathfrak{P}: \operatorname{graph}(\mathfrak{F}) \rightarrow$ $\mathbb{R}$. Preliminarily, we examine the continuity properties of $\partial_{t} \mathcal{J}$. The following result is a consequence of [MaM09, Prop. 4.4] (see also [FrM06]), combined with (6.13).

Lemma 6.3. Assume (3.L), let $W$ be frame-indifferent and fulfill $\left(3 . \mathrm{W}_{1}\right)-\left(3 . \mathrm{W}_{3}\right)$ and (6.W. $\mathrm{W}_{3}$, and let $g_{\mathrm{Dir}}$ comply with (6.4). Then, we have

$$
\left.\begin{array}{l}
t_{n} \rightarrow t, y_{n} \rightarrow y \text { in } W^{1, q_{\Phi}}\left(\Omega ; \mathbb{R}^{d}\right), \\
P_{n} \rightarrow P \text { in } W^{1, q_{\mathrm{G}}}\left(\Omega ; \mathbb{R}^{d \times d}\right), \\
\mathcal{J}\left(t_{n}, y_{n}, P_{n}\right) \rightarrow \mathcal{J}(t, y, P)<\infty
\end{array}\right\} \Longrightarrow \partial_{t} \mathcal{J}\left(t_{n}, y_{n}, P_{n}\right) \rightarrow \partial_{t} \mathcal{J}(t, y, P)
$$

Therefore, let $\left(t_{n}\right)_{n} \subset[0, T],\left(P_{n}\right)_{n} \subset X=L^{p}\left(\Omega ; \mathbb{R}^{d \times d}\right)$, and $\left(\Xi_{n}\right)_{n} \subset X^{*}=L^{p^{\prime}}\left(\Omega ; \mathbb{R}^{d \times d}\right)$ with $\Xi_{n} \in \mathfrak{F}\left(t_{n}, P_{n}\right)$ for all $n \in \mathbb{N}$ converge to $t, P, \Xi$ as in (4.43). Thus $\mathfrak{P}\left(t_{n}, P_{n}, \Xi_{n}\right)=$ $\partial_{t} \mathcal{J}\left(t_{n}, \tilde{y}_{n}, P_{n}\right)$ for some $\tilde{y}_{n} \in \mathfrak{R}\left(t_{n}, P_{n}, \Xi_{n}\right)$. The very same arguments as in Step 5 in the proof of Lemma 4.9 yield that, up to a further (not relabeled) subsequence $\tilde{y}_{n}$ weakly converges in $W^{q_{\Phi}}\left(\Omega ; \mathbb{R}^{d}\right)$ to some $\tilde{y} \in \mathfrak{R}(t, P, \Xi)$. Thanks to Lemma 6.3 we have

$$
\lim _{n \rightarrow \infty} \mathfrak{P}\left(t_{n}, P_{n}, \Xi_{n}\right)=\lim _{n \rightarrow \infty} \partial_{t} \mathcal{J}\left(t_{n}, y_{n}, P_{n}\right)=\partial_{t} \mathcal{J}(t, y, P) \leq \mathfrak{P}(t, P, \Xi),
$$


whence the upper semicontinuity of $\mathfrak{P}$. This concludes the proof of the closedness $2 . \mathrm{E}_{5}$.

Finally, combining the arguments in the proof of Lemma 5.4 with property (6.13) it can be checked that the CRI (2.15) holds for the regularized energy $\mathcal{E}_{\eta}$ also in the case of time-dependent Dirichlet boundary conditions. Thus, the existence of EDI and EDB solutions follows as in Section 4 .

\section{References}

[AGS05] L. Ambrosio, N. Gigli, and G. Savaré. Gradient flows in metric spaces and in the space of probability measures. Lectures in Mathematics ETH Zürich. Birkhäuser Verlag, Basel, 2005.

[Amb95] L. Ambrosio. Minimizing movements. Rend. Accad. Naz. Sci. XL Mem. Mat. Appl. (5), 19, 191-246, 1995.

[Ant95] S. S. Antman. Nonlinear problems of elasticity, volume 107 of Applied Mathematical Sciences. Springer-Verlag, New York, 1995.

[Att84] H. Aтtouch. Variational Convergence of Functions and Operators. Pitman Advanced Publishing Program. Pitman, 1984.

[Bal77] J. M. BALL. Convexity conditions and existence theorems in nonlinear elasticity. Arch. Rational Mech. Anal., 63(4), 337-403, 1977.

[Bal84] J. M. BALL. Minimizers and the Euler-Lagrange equations. In Trends and applications of pure mathematics to mechanics (Palaiseau, 1983), volume 195 of Lecture Notes in Phys., pages 1-4. Springer, Berlin, 1984.

[Bal02] J. M. BALl. Some open problems in elasticity. In P. Newton, P. Holmes, and A. Weinstein, editors, Geometry, Mechanics, and Dynamics, pages 3-59. Springer, New York, 2002.

[BOP91] P. Bauman, N. C. Owen, and D. Phillips. Maximum principles and a priori estimates for a class of problems from nonlinear elasticity. Ann. Inst. H. Poincaré Anal. Non Linéaire, 8(2), $119-157,1991$.

[Bre15] Y. Brenier. Connections between optimal transport, combinatorial optimization and hydrodynamics. ESAIM Math. Model. Numer. Anal., 49(6), 1593-1605, 2015.

[CHM02] C. Carstensen, K. Hackl, and A. Mielke. Non-convex potentials and microstructures in finite-strain plasticity. Proc. Royal Soc. London Ser. A, 458(2018), 299-317, 2002.

[Cia82] P. G. Ciarlet. Introduction à l'analyse numérique matricielle et à l'optimisation. Collection Mathématiques Appliquées pour la Maîtrise. [Collection of Applied Mathematics for the Master's Degree]. Masson, Paris, 1982.

[CoO05] S. Conti and M. OrTiz. Dislocation microstructures and the effective behavior of single crystals. Arch. Ration. Mech. Anal., 176(1), 103-147, 2005.

[CoT05] S. Conti and F. TheIl. Single-slip elastoplastic microstructures. Arch. Rational Mech. Anal., $178,125-148,2005$.

[DaL10] G. Dal Maso and G. Lazzaroni. Quasistatic crack growth in finite elasticity with noninterpenetration. Ann. Inst. H. Poinc. Anal. Non Lin., 27(1), 257-290, 2010.

[DFT05] G. Dal Maso, G. Francfort, and R. Toader. Quasistatic crack growth in nonlinear elasticity. Arch. Rational Mech. Anal., 176, 165-225, 2005.

[DMT80] E. De Giorgi, A. Marino, and M. Tosques. Problems of evolution in metric spaces and maximal decreasing curve. Atti Accad. Naz. Lincei Rend. Cl. Sci. Fis. Mat. Natur. (8), 68(3), 180-187, 1980.

[Eis79] G. EISEN. A selection lemma for sequences of measurable sets, and lower semicontinuity of multiple integrals. Manuscripta Math., 27, 73-79, 1979.

[Fré02] M. FrÉmond. Non-Smooth Thermomechanics. Springer-Verlag, Berlin, 2002. 
[FrM06] G. Francfort and A. Mielke. Existence results for a class of rate-independent material models with nonconvex elastic energies. J. reine angew. Math., 595, 55-91, 2006.

[HaN75] B. Halphen and Q. S. Nguyen. Sur les matériaux standards généralisés. J. Mécanique, 14, 39-63, 1975.

[HHM12] K. Hackl, S. Heinz, and A. Mielke. A model for the evolution of laminates in finite-strain elastoplasticity. Z. angew. Math. Mech. (ZAMM), 92(11-12), 888-909, 2012.

[Iof77] A. D. Ioffe. On lower semicontinuity of integral functionals. I. SIAM J. Control Optimization, $15(4), 521-538,1977$.

[KZM10] D. Knees, C. Zanini, and A. Mielke. Crack growth in polyconvex materials. Physica D, 239, 1470-1484, 2010.

[Lee69] E. H. LEE. Elastic-plastic deformation at finite strains. J. Applied Mechanics, 36, 1-6, 1969.

[MaH94] J. Marsden and T. J. Hughes. Mathematical foundations of elasticity. Dover Publications Inc., New York, 1994. Corrected reprint of the 1983 original.

[MaM09] A. MAinik and A. Mielke. Global existence for rate-independent gradient plasticity at finite strain. J. Nonlinear Sci., 19(3), 221-248, 2009.

[Mau92] G. A. Maugin. The Thermomechanics of Plasticity and Fracture. Cambridge University Press, Cambridge, 1992.

[Mie03a] C. Miene. Computational micro-to-macro transitions for discretized micro-structures of heterogeneous materials at finite strains based on the minimization of averaged incremental energy. Comput. Methods Appl. Mech. Engrg., 192(5-6), 559-591, 2003.

[Mie03b] A. MiELKE. Energetic formulation of multiplicative elasto-plasticity using dissipation distances. Contin. Mech. Thermodyn., 15, 351-382, 2003.

[Mie11] A. Mielke. Formulation of thermoelastic dissipative material behavior using GENERIC. Contin. Mech. Thermodyn., 23(3), 233-256, 2011.

[Mie16] A. Mielke. On evolutionary $\Gamma$-convergence for gradient systems (ch.3). In A. Muntean, J. Rademacher, and A. Zagaris, editors, Macroscopic and Large Scale Phenomena: Coarse Graining, Mean Field Limits and Ergodicity, Lecture Notes in Applied Math. Mechanics Vol. 3, pages 187-249. Springer, 2016. Proc. of Summer School in Twente University, June 2012.

[MiM06] A. Mielke and S. MüLLER. Lower semicontinuity and existence of minimizers for a functional in elastoplasticity. ZAMM Z. angew. Math. Mech., 86(3), 233-250, 2006.

[MiR15a] A. Mielke and T. RoubíčEK. Rate-independent elastoplasticity at finite strain and its numerical approximation. Math. Models Meth. Appl. Sci. $\left(M^{3} A S\right), 2015$. To appear. WIAS preprint 2210 .

[MiR15b] A. Mielke and T. RoubíčEk. Rate-Independent Systems: Theory and Application. Applied Mathematical Sciences, Vol. 193. Springer-Verlag New York, 2015.

[MOŞ14] A. Mielke, C. Ortner, and Y. ŞEnGül. An approach to nonlinear viscoelasticity via metric gradient flows. SIAM J. Math. Analysis, 46(2), 1317-1347, 2014.

[MRS13] A. Mielke, R. Rossi, and G. Savaré. Nonsmooth analysis of doubly nonlinear evolution equations. Calc. Var. Part. Diff. Eqns., 46(1-2), 253-310, 2013.

[MüA91] H.-B. Mühlhaus and E. C. Aifantis. A variational principle for gradient plasticity. Internat. J. Solids Structures, 28(7), 845-857, 1991.

[OrR99] M. Ortiz and E. Repetto. Nonconvex energy minimization and dislocation structures in ductile single crystals. J. Mech. Phys. Solids, 47(2), 397-462, 1999.

[OrS99] M. Ortiz and L. StAiniER. The variational formulation of viscoplastic constitutive updates. Comput. Methods Appl. Mech. Engrg., 171(3-4), 419-444, 1999.

[ORS00] M. Ortiz, E. Repetto, and L. Stainier. A theory of subgrain dislocation structures. J. Mech. Physics Solids, 48, 2077-2114, 2000. 
[Res67] Y. REsheTNYAK. On the stability of conformal maps in multidimensional spaces. Siberian Math. J., 8, 69-85, 1967.

[RMS08] R. Rossi, A. Mielke, and G. Savaré. A metric approach to a class of doubly nonlinear evolution equations and applications. Ann. Sc. Norm. Super. Pisa Cl. Sci. (5), VII(1), 97-169, 2008.

[RoS06] R. Rossi and G. SAvaré. Gradient flows of non convex functionals in Hilbert spaces and applications. ESAIM Control Optim. Calc. Var., 12, 564-614, 2006.

[Str90] M. Struwe. Variational Methods. Applications to Nonlinear Partial Differential Equations and Hamiltonian Systems. Springer-Verlag, Berlin, 1990.

[ZR*06] N. ZaAfarani, D. RaAbe, R. N. Singh, F. Roters, and S. Zaefferer. Three-dimensional investigation of the texture and microstructure below a nanoindent in a $\mathrm{Cu}$ single crystal using 3D EBSD and crystal plasticity finite element simulations. Acta Materialia 54 (2006) 18631876, 54, $18631876,2006$. 